\title{
WELL-POSED STOKES/BRINKMAN AND STOKES/DARCY COUPLING REVISITED WITH NEW JUMP INTERFACE CONDITIONS
}

\author{
PhILIPPe ANGOT ${ }^{1}$
}

\begin{abstract}
The global well-posedness in time is proved, with no restriction on the size of the data, for the Stokes/Brinkman and Stokes/Darcy coupled flow problems with new jump interface conditions recently derived in [11] by asymptotic modelling and shown to be physically relevant. These original conditions include jumps of both stress and tangential velocity vectors at the fluid-porous interface. They can be viewed as generalizations for the multi-dimensional flow of Beavers \& Joseph's jump condition of tangential velocity and Ochoa-Tapia \& Whitaker's jump condition of shear stress. Therefore, they are different from those most commonly used in the literature. The case of Saffman's approximation is also studied, but with a force balance for the cross-flow including the Darcy drag and inducing a law of pressure jump different from the usual one. The proof of these results follows the general framework briefly introduced in $[7,8]$ for the steady flow.
\end{abstract}

1991 Mathematics Subject Classification. 35Q30, 35Q35, 65M85, 76D03, 76D05, 76D07, 76S05.

Revised version, November 14, 2017.

\section{INTRODUCTION}

This paper is devoted to the well-posedness analysis of three macroscopic models for the computation of the unsteady and incompressible viscous flow in a domain composed of a pure fluid region and a fluid-saturated porous medium covering the whole range of porosity (volume fraction of fluid) $0<\phi<1$. Such problems have been the subject of numerous published articles during the past decades, e.g. studying natural convection in fluid-porous systems with different boundary conditions at the fluid-porous interface. This justifies the interest of this problem for both theoretical studies and/or applications to environmental, biological, engineering sciences or industrial configurations. More precisely, the present study considers the Stokes equation in the fluid domain and either Brinkman's law (for large porosity) or Darcy's law (for lower porosity) in the porous medium coupled with interface conditions including jumps of both the stress and tangential velocity vectors.

In the pioneering work [16] fifty years ago, Beavers \& Joseph (1967) introduced semi-empirically the jump condition of the tangential velocity for the Stokes/Darcy 1-D channel flow, which was recently shown in [22] by multi-scale homogenization. Later in [56,57], Ochoa-Tapia \& Whitaker (1995) have shown by upscaling the shear stress jump condition for the Stokes/Brinkman 1-D channel flow using the method of volume averaging [68]. Still using this method for the same 1-D channel flow, Valdés-Parada et al. (2013) have shown in [65] jump

Keywords and phrases: Fluid-porous coupled flow; Stokes/Brinkman model; Stokes/Darcy model; jump interface conditions; stress vector jump; tangential velocity jump

1 Aix-Marseille Université, CNRS, Centrale Marseille

Institut de Mathématiques de Marseille (UMR-7373)

13453 Marseille cedex 13, France

(c) EDP Sciences, SMAI 1999 
interface conditions for both the shear stress and tangential velocity. Very recently, Angot et al. (2017) derived by a physically relevant asymptotic modelling in [11] new jump interface conditions which generalize all these conditions for the two- or three-dimensional flow.

Concerning the mathematical or numerical analysis of such coupled flows, many papers have been published in the last fifteen years and it is not possible to cite all of them; see e.g. $[12,18,32,33,36,37,46,58,59]$ and the references therein. However, almost all of them only study the Stokes/Darcy problem with Saffman's approximation (1971) [63] of the Beavers-Joseph condition and no stress jump. Then, the tangential velocity jump is not explicitly included in the interface conditions and the Darcy drag is in fact neglected in the balance of forces by assuming the continuity of the stress vector. Indeed, one major difficulty is to propose a variational problem giving a sense to the velocity jump since the Darcy filtration velocity does not generally belong to the space $\boldsymbol{H}_{\text {rot }}$ and is not better than in the space $\boldsymbol{H}_{\text {div }}$ and thus, has no tangential trace on the interface. In $[26,27]$, the velocity jump was considered but the solvability analysis is made owing to the hypothesis of smallness of the slip coefficient $\alpha_{b j} \geq 0$ of Beavers-Joseph. Using the general framework introduced in [7], the well-posedness is proved in [8] for the steady Stokes/Darcy problem with the complete Beavers-Joseph jump condition whatever $\alpha_{b j} \geq 0$.

Hence, the main highlights of the present work is to deal with the original interface conditions derived in [11] which include jumps of both the stress and tangential velocity vectors. Then, the complete analysis of well-posedness globally in time is carried out for three fluid-porous unsteady flow problems with no smallness hypothesis on the size of data and no extra regularity assumptions than the natural ones.

The paper is organized as follows. Section 1 describes the flow models and details the different set of new jump interface conditions issued from [11] and studied further. In Section 2, the well-posedness of the unsteady Stokes/Brinkman problem is stated. The solvability analysis of the unsteady Stokes/Darcy system is carried out in Section 3, whereas a simplified case is studied in Section 4.

\section{Fluid-POROUS INCOMPRESSIBLE VISCOUS FLOW MODELS}

In this section, we describe the models of the incompressible viscous flow at a fluid-porous interface and we discuss the jump interface conditions used further for the analysis of the corresponding coupled problems.

\section{Notations and definitions}

Let $\Omega \subset \mathbb{R}^{d}$ ( $d=2$ or 3 in practice) be an open bounded and connected set with Lipschitz continuous boundary $\Gamma:=\partial \Omega$ and $\boldsymbol{\nu}$ be the outward unit normal vector on $\Gamma$. The domain $\Omega$ is composed of two disjoint connected subdomains, the fluid domain $\Omega_{f}$ and the porous domain $\Omega_{p}$ separated by a Lipschitz continuous interface $\Sigma \subset \mathbb{R}^{d-1}$ such that $\Omega=\Omega_{f} \cup \Sigma \cup \Omega_{p}$, as shown in Figure 1. For the sake of simplicity to avoid some technicalities to define the traces on $\Sigma$, we restrict to the case where the porous domain $\Omega_{p}$ is entirely bordered by the closed surface $\Sigma$ and we have $\partial \Omega_{p}=\Sigma$ whereas $\partial \Omega_{f}=\Gamma \cup \Sigma$. The extension to several closed interfaces is straightforward. The case of more general situations when $\Sigma$ cuts $\Gamma$ can be treated as well but it is more technical and we refer to [40] for the trace theory and also to [36] where a suitable functional setting is clearly described. Let $\boldsymbol{n}$ be the unit normal vector on the interface $\Sigma$ arbitrarily oriented from $\Omega_{p}$ to $\Omega_{f}$, and let $\left\{\boldsymbol{\tau}_{j}\right\}$ for $1 \leq j \leq d-1$, be an orthonormal set of unit vectors on the tangent plane to $\Sigma$.

We use the standard notations for the Lebesgue and Sobolev spaces, e.g. [20,54]. In particular, $\|\cdot\|_{0, \Omega}$ denotes the $L^{2}(\Omega)$-norm, $\|\cdot\|_{1, \Omega}$ the $H^{1}(\Omega)$-norm, $\|\cdot\|_{-1, \Omega}$ for the $H^{-1}(\Omega)$-norm, $(., .)_{0, \Omega}$ for the $L^{2}(\Omega)$-inner product, and $\langle., .\rangle_{-1, \Omega}$ for the duality pairing between $H^{-1}(\Omega)$ and $H_{0}^{1}(\Omega)$ or $\langle., .\rangle_{-1 / 2, \Sigma}$ for the duality pairing between $H^{-1 / 2}(\Sigma)$ and $H^{1 / 2}(\Sigma)$. We also define the Hilbert spaces below with their usual respective inner products and 


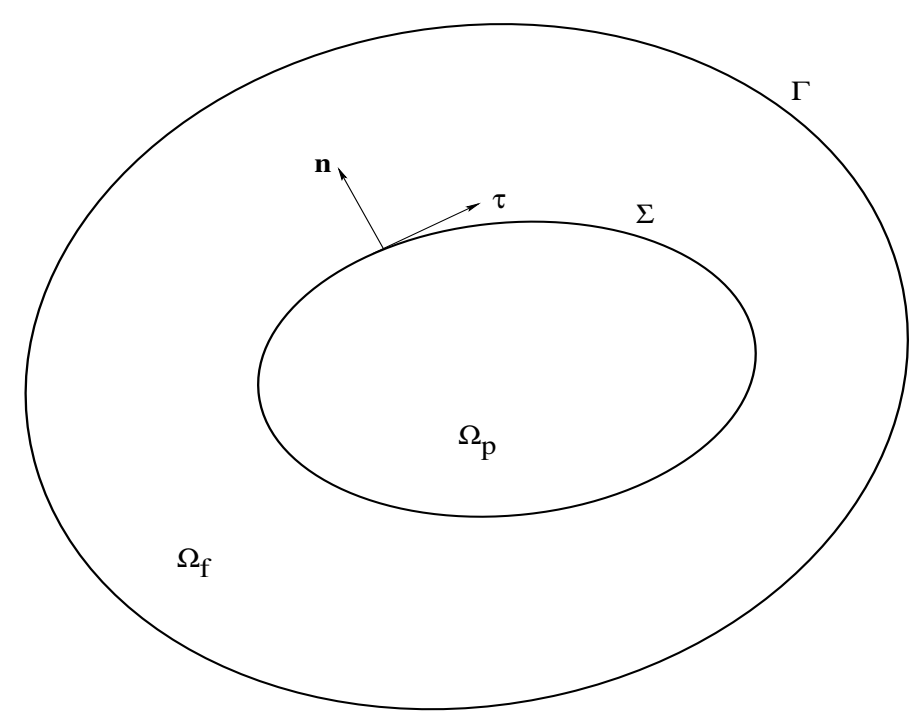

FiguRE 1. Fluid-porous configuration with a closed surface $\Sigma$ bordering the porous domain $\Omega_{p}$.

associated norms, e.g. $[19,35,64]$ :

$$
\begin{array}{lr}
\boldsymbol{H}_{\text {div }}(\Omega)=\left\{\boldsymbol{u} \in L^{2}(\Omega)^{d} ; \boldsymbol{\nabla} \cdot \boldsymbol{u} \in L^{2}(\Omega)\right\}, & \boldsymbol{H}_{\text {rot }}(\Omega)=\left\{\boldsymbol{u} \in L^{2}(\Omega)^{d} ; \boldsymbol{\nabla} \times \boldsymbol{u} \in L^{2}(\Omega)^{d}\right\} \\
\boldsymbol{H}=\left\{\boldsymbol{u} \in L^{2}(\Omega)^{d} ; \boldsymbol{\nabla} \cdot \boldsymbol{u}=0, \boldsymbol{u} \cdot \boldsymbol{\nu}=0 \text { on } \Gamma\right\}, & \boldsymbol{V}=\left\{\boldsymbol{u} \in H_{0}^{1}(\Omega)^{d} ; \boldsymbol{\nabla} \cdot \boldsymbol{u}=0\right\} \\
H_{0 \Gamma}^{1}\left(\Omega_{f}\right)^{d}=\left\{\boldsymbol{u} \in H^{1}\left(\Omega_{f}\right)^{d} ; \boldsymbol{u}=0 \text { on } \Gamma\right\}, & L_{0}^{2}(\Omega)=\left\{q \in L^{2}(\Omega) ; \int_{\Omega} q \mathrm{~d} x=0\right\} .
\end{array}
$$

For any quantity $\psi$ defined all over $\Omega$, the restrictions on $\Omega_{f}$ or $\Omega_{p}$ are respectively denoted by $\psi^{f}:=\psi_{\mid \Omega_{f}}$ and $\psi^{p}:=\psi_{\mid \Omega_{p}}$. For a function $\psi$ having a jump on $\Sigma$, let $\psi^{-}:=\psi_{\mid \Sigma}^{p}$ and $\psi^{+}:=\psi_{\mid \Sigma}^{f}$ be the traces of $\psi^{p}$ and $\psi^{f}$ on each side of $\Sigma$ (at least defined in a weak sense), respectively. Following the framework defined in $[5,6]$ for scalar elliptic problems with jump interface conditions or its extension to vector problems in [7], let us choose as reduced variables at the interface $\Sigma$, the jump of $\psi$ on $\Sigma$ oriented by $\boldsymbol{n}$ and the arithmetic mean of traces of $\psi$ defined by :

$$
\llbracket \psi \rrbracket_{\Sigma}:=\psi^{+}-\psi^{-}=\left(\psi^{f}-\psi^{p}\right)_{\mid \Sigma}, \quad \text { and } \quad \bar{\psi}_{\Sigma}:=\frac{1}{2}\left(\psi^{+}+\psi^{-}\right)=\frac{1}{2}\left(\psi^{f}+\psi^{p}\right)_{\mid \Sigma} .
$$

Thus we have also :

$$
\psi_{\mid \Sigma}^{f}:=\psi^{+}=\bar{\psi}_{\Sigma}+\frac{1}{2} \llbracket \psi \rrbracket_{\Sigma}, \quad \text { and } \quad \psi_{\mid \Sigma}^{p}:=\psi^{-}=\bar{\psi}_{\Sigma}-\frac{1}{2} \llbracket \psi \rrbracket_{\Sigma}
$$

\subsection{Fluid-porous incompressible viscous flow}

Let us consider the unsteady incompressible Stokes equations in $\Omega_{f}$ for a Newtonian fluid of constant density $\rho>0$ and dynamic viscosity $\mu>0,(\boldsymbol{v}, p)$ denoting the velocity and pressure fields respectively, and $\boldsymbol{f}$ represents the source force : 


$$
\begin{aligned}
& \boldsymbol{\nabla} \cdot \boldsymbol{v}=0 \quad \text { in }(0, T) \times \Omega_{f}, \\
& \rho \partial_{t} \boldsymbol{v}-\mu \boldsymbol{\Delta} \boldsymbol{v}+\nabla p=\rho \boldsymbol{f} \quad \text { in }(0, T) \times \Omega_{f} \text {, } \\
& \boldsymbol{v}=0 \quad \text { on }(0, T) \times \Gamma \text {, } \\
& \boldsymbol{v}(t=0)=\boldsymbol{v}_{0}^{f} \quad \text { in } \Omega_{f} .
\end{aligned}
$$

The porous matrix in $\Omega_{p}$ is heterogeneous, non-deformable and characterized by its porosity $0<\phi \leq 1$ (volume fraction of fluid) and permeability tensor $\boldsymbol{K}$, symmetric and uniformly bounded and positive definite, both spatially dependent but supposed to be time-independent. The characteristic length scale $\ell_{p}$ of the pore size is known to verify $\ell_{p}=\mathcal{O}(\sqrt{K})$ where $K:=\|\boldsymbol{K}\|_{L^{\infty}\left(\Omega_{p}\right)}$ and $\ell_{p} \ll L$ where $L$ is the macroscopic length scale. In the non-homogeneous fluid-saturated porous region $\Omega_{p}$, we consider the generalized Brinkman equation with variable porosity $\phi$ where the effective viscosity $\widetilde{\mu}$ is equal to $\widetilde{\mu}:=\mu / \phi$; see [68, Chap. 4] :

$$
\begin{aligned}
& \boldsymbol{\nabla} \cdot \boldsymbol{v}=0 \quad \text { in }(0, T) \times \Omega_{p}, \\
& \rho \partial_{t} \boldsymbol{v}-\nabla \cdot(\widetilde{\mu} \nabla \boldsymbol{v})+\mu \boldsymbol{K}^{-1} \boldsymbol{v}+\nabla p=\rho \boldsymbol{f} \quad \text { in }(0, T) \times \Omega_{p}, \quad \text { with } \widetilde{\mu}:=\frac{\mu}{\phi} \\
& \boldsymbol{v}(t=0)=\boldsymbol{v}_{0}^{p} \quad \text { in } \Omega_{p} .
\end{aligned}
$$

In the present macroscopic description of flow in a permeable medium, $\boldsymbol{v}$ denotes the filtration velocity, i.e. averaged over the whole representative elementary volume, whereas $p$ is averaged only over the fluid-pore. The permeability tensor is in fact a function of porosity and porosity gradient like $\boldsymbol{K}(\phi, \nabla \phi)$ which depends on the local structure of the porous medium. The explicit variation with $\nabla \phi$ can be generally neglected. In the homogeneous porous medium (with a constant porosity and permeability), the original Brinkman equation $[23,24]$ is shown to actually govern the macroscopic flow in a porous medium with high porosity values (about $\phi \geq 0.9$ ) by several upscaling procedures: deterministic homogenization with multi-scale asymptotic expansions $[2,21,47,48]$, stochastic homogenization for random porous media [60,61] or volume averaging method [68]. For $\phi=1$ or $\widetilde{\mu}^{f}=\mu$ and $\boldsymbol{K}=1 / \varepsilon \boldsymbol{I} \rightarrow+\infty$ when $\varepsilon \rightarrow 0$, it is shown in [4] that Eq. (6) tends to the Stokes equation. This is also in agreement with the upscaling results since the solid inclusions in the porous region become too small to influence the Stokes flow.

Let us introduce the pseudo-stress tensor, although the complete stress tensor can be considered as well :

$$
\boldsymbol{\sigma}(\boldsymbol{v}, p):=\widetilde{\mu} \nabla \boldsymbol{v}-p \boldsymbol{I}=\frac{\mu}{\phi} \nabla \boldsymbol{v}-p \boldsymbol{I} \quad \text { in } \Omega,
$$

where $\phi=1$ and thus $\widetilde{\mu}=\mu$ in $\Omega_{f}$. Then, the divergential form of Eq. (6) reads :

$$
\rho \partial_{t} \boldsymbol{v}-\boldsymbol{\nabla} \cdot \boldsymbol{\sigma}(\boldsymbol{v}, p)+\mu \boldsymbol{K}^{-1} \boldsymbol{v}=\rho \boldsymbol{f} \quad \text { in }(0, T) \times \Omega_{p} .
$$

When the porosity is smaller $(0<\phi \leq 0.9)$, it is well-known that the Brinkman viscous term in (6) can be neglected; see e.g. [68, Chap. 4]. Then, the usual Darcy equation $[15,66]$ is obtained :

$$
\rho \partial_{t} \boldsymbol{v}+\mu \boldsymbol{K}^{-1} \boldsymbol{v}+\nabla p=\rho \boldsymbol{f} \quad \text { in }(0, T) \times \Omega_{p} .
$$

It is now also classical to derive Darcy's law by deterministic homogenization e.g. [3,48] or probabilistic upscaling $[17,62]$. Formally, Eq. (6) gives (10) by letting $\widetilde{\mu}=0$, see Remark 1.1. This is rigorously proved in [8] using the vanishing viscosity method when $\widetilde{\mu} \rightarrow 0$ and in [10] by calculating the viscous boundary layer. 


\section{Remark 1.1 (From the unsteady Brinkman model to steady Darcy model).}

In the homogenization theory [2,3,21,47,60], only one model, either Brinkman or Darcy, is selected through the limit process according to the chosen scaling in the multiscale asymptotic expansion. On the contrary, the volume averaging method [39,68] allows us to keep all the terms and then retain the more significant ones through physically meaningful approximations.

In the Brinkman equation (6) or (9) and the Darcy equation (10), the time evolution term should be correctly written as : $\rho / \phi \partial_{t} \boldsymbol{v}, \boldsymbol{v}$ being the filtration velocity, e.g. [67].

It is useful to compare the order of magnitude of the ratio $R_{\text {evol }}(\phi)$ between the term of velocity time derivative over Darcy's drag term. By taking $V$ as the reference velocity and choosing $L / V$ as the reference time scale, we have:

$$
R_{\text {evol }}(\phi)=\mathcal{O}\left(R e \frac{D a(\phi)}{\phi}\right), \quad \text { with } \quad R e:=\frac{\rho V L}{\mu}, \quad D a(\phi):=\frac{K(\phi)}{L^{2}} .
$$

Since the inertial effects are here neglected, we have for the Reynolds number Re $\ll 1$. In many real configurations where $L$ is large enough, we have also for the Darcy number Da $\ll 1$. More precisely, it appears in Appendix $A$ using classical correlations $K(\phi)$ for granular or fibrous media that $R_{\text {evol }}(\phi) \ll 1$, except when the porosity $\phi$ is close to 1 . Hence, the time evolution term can be most often neglected, although we shall keep further the unsteady form of these equations in $\Omega_{p}$ for the sake of mathematical convenience in the solvability analysis. However, the steady Darcy model is also considered in the coupled problem (39,34) and studied in Corollary 3.4.

In the same spirit, the order of magnitude of the ratio $R_{B / D}(\phi)$ between the Brinkman viscous diffusion term over Darcy's drag reads :

$$
R_{B / D}(\phi)=\mathcal{O}\left(\frac{D a(\phi)}{\phi}\right), \quad \text { with } \quad D a(\phi):=\frac{K(\phi)}{L^{2}}
$$

As shown in Fig. 3 in Appendix A, the Brinkman viscous term can be neglected with respect to Darcy's term when $\phi \leq 0.9$. Hence, within a suitable rescaling as in homogenization theory [2, 3], it formally amounts to take the limit $\widetilde{\mu} \rightarrow 0$ in the Brinkman equation (6) to recover Darcy's one (10) in $\Omega_{p}$. Moreover, when $\phi \rightarrow 0$ and thus $K(\phi) \rightarrow 0$ from Fig. 2, then it is proved in [4] that we get at the limit $\boldsymbol{v}^{p}=0$ in $\Omega_{p}$. When $\phi \rightarrow 1$ and thus $1 / K(\phi) \rightarrow 0$, then the Stokes equation (2) is fully recovered from the generalized Brinkman law (6); see [4].

\subsection{The jump interface conditions for Brinkman or Darcy flows}

We now detail the original jump interface conditions derived in [11] for the multi-dimensional flow by asymptotic modelling over a thin interfacial layer of thickness $d=\mathcal{O}(\sqrt{K})$ around $\Sigma$; more precisely $d \approx 20 \sqrt{K} \ll L$ $[56,65]$. They are obtained in the vector form up to first-order in $\mathcal{O}(d / L)$. The set of steady interface conditions reads as below in terms of jumps of both stress and velocity vectors and they are physically relevant to couple the Stokes flow (1-4) in $\Omega_{f}$ to the Brinkman flow (5-7) in $\Omega_{p}$ :

$$
\left\{\begin{aligned}
\llbracket \boldsymbol{v} \cdot \boldsymbol{n} \rrbracket_{\Sigma} & =0, \\
{\overline{\boldsymbol{\sigma}(\boldsymbol{v}, p) \cdot \boldsymbol{n}_{\Sigma}}}_{\boldsymbol{1}} \boldsymbol{\tau}_{j}= & \overline{\widetilde{\mu} \nabla \boldsymbol{v} \cdot \boldsymbol{n}_{\Sigma} \cdot \boldsymbol{\tau}_{j}}=\mu \frac{\alpha}{\sqrt{K}} \llbracket \boldsymbol{v} \cdot \boldsymbol{\tau}_{j} \rrbracket_{\Sigma}, \quad 1 \leq j \leq d-1 \quad \text { on }(0, T) \times \Sigma . \\
\llbracket \boldsymbol{\sigma}(\boldsymbol{v}, p) \cdot \boldsymbol{n} \rrbracket_{\Sigma} & =\frac{\mu}{\sqrt{K}} \boldsymbol{\beta} \overline{\boldsymbol{v}}_{\Sigma}+\boldsymbol{h},
\end{aligned}\right.
$$

The null-jump of the normal velocity $\llbracket \boldsymbol{v} \cdot \boldsymbol{n} \rrbracket_{\Sigma}=0$ across $\Sigma$ is classically assumed by many authors without justification. In fact, this is only an approximation up to $\mathcal{O}(d / L)$ obtained by averaging the mass conservation equation $\boldsymbol{\nabla} \cdot \boldsymbol{v}=0$ over the interfacial layer and neglecting all the tangential derivatives along $\Sigma$. For instance, such an approximation is not made to derive asymptotic models in fractured porous media [9].

The two other equations in (12) are new. The second equation in (12) corresponds to the average of the viscous pseudo-stress vector and the dimensionless jump coefficient $0<\alpha \in L^{\infty}(\Sigma)$ can be related to the slip 
coefficient of Beavers \& Joseph [16], originally introduced for the 1-D channel flow. The last equation in (12) is obtained by averaging the momentum transport equation over the interfacial layer and it corresponds to the balance of forces over the interface $\Sigma$. Here, $\boldsymbol{h}$ is a given surface force on $\Sigma$ usually taken as $\boldsymbol{h}=0$ in many applications, and $\boldsymbol{\beta}$ denotes the so-called Darcy friction tensor which is dimensionless, symmetric and uniformly bounded and positive definite. The dimensionless coefficients $\alpha$ and $\boldsymbol{\beta}$ depend on the local micro-structure of the interfacial region and should be estimated from experimental data or direct numerical simulations at the porescale in ordered or random media. We refer to [11] for the details concerning the derivation and the connections with other interface conditions available in the literature: Beavers \& Joseph (1967) [16], Saffman (1971) [63], Ochoa-Tapia \& Whitaker (1995) [56,57], or generalizations including both stress and velocity jumps [65] and for multi-dimensional flows, those devised in $[7,8]$.

For the sake of clarity, let us write the tangential and normal components of Eq. (12) in the 2-D case, $\tau$ being any unit tangent vector on $\Sigma$ and defining the tensor $\boldsymbol{\beta}$ as :

$$
\boldsymbol{\beta}:=\left(\begin{array}{cc}
\beta_{\tau} & \beta_{\tau n} \\
\beta_{\tau n} & \beta_{n}
\end{array}\right), \quad \text { Darcy friction tensor on } \Sigma .
$$

The coefficients $\beta_{\tau}, \beta_{\tau n}, \beta_{n} \in L^{\infty}(\Sigma)$ are dimensionless and such that $\beta_{\tau}, \beta_{n}>0$ on $\Sigma$. Then, Eq. (12) also reads taking $\boldsymbol{h}=0$ :

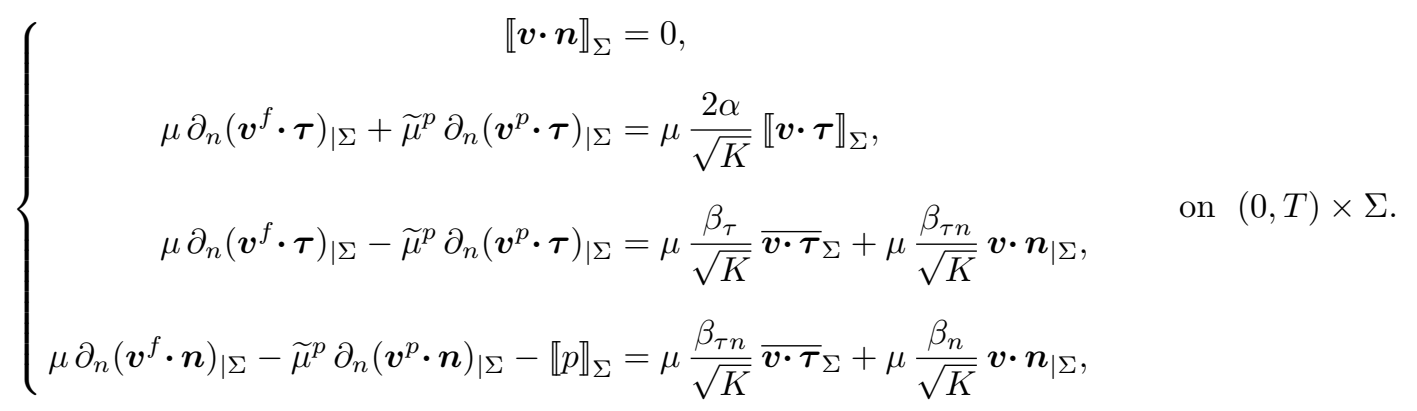

Hence, we get a generalization for multi-dimensional flow of the shear stress jump interface condition of OchoaTapia \& Whitaker [56] which was originally derived by the volume averaging method for the 1-D channel flow with $\boldsymbol{v} \cdot \boldsymbol{n}_{\mid \Sigma}=0$ and assuming $\llbracket \boldsymbol{v} \cdot \boldsymbol{\tau} \rrbracket_{\Sigma}=0$ and thus $\overline{\boldsymbol{v} \cdot \boldsymbol{\tau}_{\Sigma}}=\boldsymbol{v} \cdot \boldsymbol{\tau}_{\mid \Sigma}$. This gives the scaling of $\beta_{\tau}=\beta_{\text {otw }}=\mathcal{O}(1)$ from the experimental data in [57].

When the flow in the porous medium is to be governed by Darcy's law for a lower range of porosity, the set of jump interface conditions (12) reduces as below by formally letting $\widetilde{\mu} \rightarrow 0$ to couple the Stokes flow (1-4) in $\Omega_{f}$ to the Darcy flow $(5,10,7)$ in $\Omega_{p}$ :

$$
\left\{\begin{aligned}
\llbracket \boldsymbol{v} \cdot \boldsymbol{n} \rrbracket_{\Sigma} & =0, \\
\mu\left(\nabla \boldsymbol{v}^{f} \cdot \boldsymbol{n}_{\mid \Sigma}\right) \cdot \boldsymbol{\tau}_{j} & =\mu \frac{2 \alpha}{\sqrt{K}} \llbracket \boldsymbol{v} \cdot \boldsymbol{\tau}_{j} \rrbracket_{\Sigma}, \quad 1 \leq j \leq d-1 \quad \text { on }(0, T) \times \Sigma . \\
\mu \nabla \boldsymbol{v}^{f} \cdot \boldsymbol{n}_{\mid \Sigma}-\llbracket p \rrbracket_{\Sigma} \boldsymbol{n} & =\frac{\mu}{\sqrt{K}} \boldsymbol{\beta} \overline{\boldsymbol{v}}_{\Sigma}+\boldsymbol{h},
\end{aligned}\right.
$$

For instance in the 2-D case with $\boldsymbol{h}=0$, Eq. (14) reads : 


$$
\left\{\begin{aligned}
\llbracket \boldsymbol{v} \cdot \boldsymbol{n} \rrbracket_{\Sigma} & =0 \\
\mu \partial_{n}\left(\boldsymbol{v}^{f} \cdot \boldsymbol{\tau}\right)_{\mid \Sigma} & =\mu \frac{2 \alpha}{\sqrt{K}} \llbracket \boldsymbol{v} \cdot \boldsymbol{\tau} \rrbracket_{\Sigma}=\mu \frac{2 \alpha}{\sqrt{K}}\left(\boldsymbol{v}^{f}-\boldsymbol{v}^{p}\right)_{\mid \Sigma} \cdot \boldsymbol{\tau}, \\
\mu \partial_{n}\left(\boldsymbol{v}^{f} \cdot \boldsymbol{\tau}\right)_{\mid \Sigma} & =\mu \frac{\beta_{\tau}}{\sqrt{K}} \overline{\boldsymbol{v} \cdot \boldsymbol{\tau}_{\Sigma}+\mu \frac{\beta_{\tau n}}{\sqrt{K}} \boldsymbol{v} \cdot \boldsymbol{n}_{\mid \Sigma},} \quad \text { on }(0, T) \times \Sigma . \\
\mu \partial_{n}\left(\boldsymbol{v}^{f} \cdot \boldsymbol{n}\right)_{\mid \Sigma}-\llbracket p \rrbracket_{\Sigma} & =\mu \frac{\beta_{\tau n}}{\sqrt{K}} \overline{\boldsymbol{v} \cdot \boldsymbol{\tau}_{\Sigma}}+\mu \frac{\beta_{n}}{\sqrt{K}} \boldsymbol{v} \cdot \boldsymbol{n}_{\mid \Sigma},
\end{aligned}\right.
$$

Hence, the semi-empirical Beavers \& Joseph (1967) jump interface condition [16], originally introduced for the 1-D channel or shear flow such that $\boldsymbol{v} \cdot \boldsymbol{n}_{\mid \Sigma}=0$ and Darcy's law in the porous medium, is here generalized in Eq. (15) for the multi-dimensional flow. This gives the scaling of the jump coefficient $\alpha$ like: $\alpha_{b j}=2 \alpha=\mathcal{O}(1)$. The original jump condition of Beavers \& Joseph was also derived in [30] and recently proved in [22] via multiscale homogenization. We refer to $[11,13,38,55,58]$ for other studies or comments.

The last equation in (15) gives the pressure jump across $\Sigma$ that generalizes the equation derived in [45]. Besides, the pressure jump vanishes for the 1-D channel flow when $\boldsymbol{v} \cdot \boldsymbol{n}_{\mid \Sigma}=0$ and $\beta_{\tau n}=0$, which is a condition often admitted by some authors. However, the pressure jump can be non-zero even in 1-D if the tensor $\boldsymbol{\beta}$ is not diagonal. The existence of the non-zero pressure jump was recently confirmed both by homogenization in [52] and direct numerical simulations in [29].

\subsection{Simplified interface conditions for Darcy flow}

We now consider Saffman's usual approximation [63] of Beavers \& Joseph's jump interface condition which was originally introduced for the 1-D channel or shear flow when the porous flow is governed by Darcy's law with relatively small values of permeability $K$. It results that $\left|\boldsymbol{v}^{p} \cdot \boldsymbol{\tau}_{\mid \Sigma}\right| \ll\left|\boldsymbol{v}^{f} \cdot \boldsymbol{\tau}_{\mid \Sigma}\right|$ and the tangential velocity

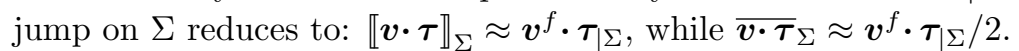

Indeed, in the present case using Darcy's law (10) and the last equation in (15) to get a reference pressure on $\Sigma$ with $d=\mathcal{O}(\sqrt{K})$ and a reference fluid velocity $V$, we have :

$$
\left|\boldsymbol{v}^{p} \cdot \boldsymbol{\tau}_{\mid \Sigma}\right|=\mathcal{O}\left(\frac{K}{\mu L} \frac{\mu \beta}{\sqrt{K}} V\right)=\mathcal{O}\left(\beta V \frac{d}{L}\right)
$$

which is negligible in front of $\left|\boldsymbol{v}^{f} \cdot \boldsymbol{\tau}_{\mid \Sigma}\right|=\mathcal{O}(V)$ up to $\mathcal{O}(d / L)$.

Besides, we assume $\boldsymbol{h}=0$ and $\left|\beta_{\tau n}\right| \ll \beta_{\tau}, \beta_{n}$, that is $\boldsymbol{\beta}$ can be approximated by a diagonal tensor.

In that case, the set of interface conditions (15) to deal with the Stokes/Darcy flow is therefore simplified and reduces with $\alpha_{b j}=2 \alpha$ to:

$$
\left\{\begin{aligned}
\llbracket \boldsymbol{v} \cdot \boldsymbol{n} \rrbracket_{\Sigma} & =0 \\
\mu \partial_{n}\left(\boldsymbol{v}^{f} \cdot \boldsymbol{\tau}\right)_{\mid \Sigma} & =\mu \frac{\alpha_{b j}}{\sqrt{K}} \boldsymbol{v}^{f} \cdot \boldsymbol{\tau}_{\mid \Sigma}, \\
\mu \partial_{n}\left(\boldsymbol{v}^{f} \cdot \boldsymbol{\tau}\right)_{\mid \Sigma} & =\mu \frac{\beta_{\tau}}{2 \sqrt{K}} \boldsymbol{v}^{f} \cdot \boldsymbol{\tau}_{\mid \Sigma}, \quad \text { on }(0, T) \times \Sigma \\
\mu \partial_{n}\left(\boldsymbol{v}^{f} \cdot \boldsymbol{n}\right)_{\mid \Sigma}-\llbracket p \rrbracket_{\Sigma} & =\mu \frac{\beta_{n}}{\sqrt{K}} \boldsymbol{v} \cdot \boldsymbol{n}_{\mid \Sigma},
\end{aligned}\right.
$$

Then, the second and third equation in (16) give both the standard Beavers-Joseph-Saffman condition and one of them becomes redundant. It reduces the number of parameters since we have : $\beta_{\tau}=\beta_{\text {otw }}=2 \alpha_{b j}=\mathcal{O}(1)$ 
and $\beta_{n}=\mathcal{O}(1)$. The classical Beavers-Joseph-Saffman condition is proved by homogenization in [43, 44]. Nevertheless, it is important to point out that Saffman's approximation does not hold anymore when the porosity and permeability are large enough or for thin fluid layers.

Remark 1.2 (Generalized Beavers-Joseph-Saffman interface conditions for multi-dimensional flow). Even with Saffman's approximation, Eq. (16) is new to deal with the Stokes/Darcy problem. Indeed, almost all the authors admit that the adhoc balance of forces in the normal direction on $\Sigma$ reads with no stress jump as:

$$
\left(p^{f}-\mu \partial_{n}\left(\boldsymbol{v}^{f} \cdot \boldsymbol{n}\right)\right)_{\mid \Sigma}=p_{\mid \Sigma}^{p}, \quad \text { i.e. also } \quad \mu \partial_{n}\left(\boldsymbol{v}^{f} \cdot \boldsymbol{n}\right)_{\mid \Sigma}=\llbracket p \rrbracket_{\Sigma}
$$

which leads to a different equation for the pressure jump by assuming so the continuity of the normal component of the stress vector on $\Sigma$. However, Eq. (17) is questionable from the physical point of view since it does not take account of the Darcy drag force for the transverse flow across the interface $\Sigma$ as in Eq. (16) with Darcy's law in $\Omega_{p}$, although this force has the same order of magnitude of the pressure force. Indeed, we have since $\beta_{n}=\mathcal{O}(1)$ :

$$
\llbracket p \rrbracket_{\Sigma}=\mathcal{O}\left(\mu \frac{V}{\sqrt{K}}\right), \quad \text { and } \quad \mu \frac{\beta_{n}}{\sqrt{K}} \boldsymbol{v} \cdot \boldsymbol{n}_{\mid \Sigma}=\mathcal{O}\left(\mu \beta_{n} \frac{V}{\sqrt{K}}\right) .
$$

Therefore, the last term of Darcy's drag is not a priori negligible and the two corresponding models are not physically compatible.

\section{Stokes/Brinkman Problem With Jump interface CONDitions}

In this part, the Stokes/Brinkman coupled flow problem with the jump interface conditions (12) is proved to be globally well-posed in time.

\subsection{Formulation of the problem and functional setting}

The problem reads as below in the time-space domain $(0, T) \times \Omega$ for any $T>0$ :

$$
\left\{\begin{array}{rlrl}
\boldsymbol{\nabla} \cdot \boldsymbol{v} & =0 & \text { in }(0, T) \times \Omega_{f} \cup \Omega_{p}, \\
\rho \partial_{t} \boldsymbol{v}-\boldsymbol{\nabla} \cdot \boldsymbol{\sigma}(\boldsymbol{v}, p)^{f} & =\rho \boldsymbol{f} & & \text { in }(0, T) \times \Omega_{f}, \\
\rho \partial_{t} \boldsymbol{v}-\boldsymbol{\nabla} \cdot \boldsymbol{\sigma}(\boldsymbol{v}, p)^{p}+\mu \boldsymbol{K}^{-1} \boldsymbol{v} & =\rho \boldsymbol{f} & & \text { in }(0, T) \times \Omega_{p}, \\
\boldsymbol{v} & =0 & & \text { on }(0, T) \times \Gamma, \\
\boldsymbol{v}(t=0) & =\boldsymbol{v}_{0} & & \text { in } \Omega,
\end{array}\right.
$$

where :

$$
\begin{aligned}
& \boldsymbol{\sigma}(\boldsymbol{v}, p):=\widetilde{\mu} \nabla \boldsymbol{v}-p \boldsymbol{I}, \quad \text { with } \quad \phi^{f}:=1, \quad \widetilde{\mu}^{f}:=\mu, \quad \widetilde{\mu}^{p}:=\frac{\mu}{\phi} \\
& \left\{\begin{aligned}
\llbracket \boldsymbol{v} \cdot \boldsymbol{n} \rrbracket_{\Sigma} & =0, \\
{\overline{\boldsymbol{\sigma}(\boldsymbol{v}, p) \cdot \boldsymbol{n}_{\Sigma}}}_{\boldsymbol{\tau}_{j}}= & \overline{\widetilde{\mu} \nabla \boldsymbol{v} \cdot \boldsymbol{n}_{\Sigma} \cdot \boldsymbol{\tau}_{j}}=\mu \frac{\alpha}{\sqrt{K}} \llbracket \boldsymbol{v} \cdot \boldsymbol{\tau}_{j} \rrbracket_{\Sigma}, \quad 1 \leq j \leq d-1 \quad \text { on }(0, T) \times \Sigma . \\
\llbracket \boldsymbol{\sigma}(\boldsymbol{v}, p) \cdot \boldsymbol{n} \rrbracket_{\Sigma} & =\frac{\mu}{\sqrt{K}} \boldsymbol{\beta} \overline{\boldsymbol{v}}_{\Sigma},
\end{aligned}\right.
\end{aligned}
$$

We have basic assumptions on the data :

$\boldsymbol{f} \in L^{2}\left(0, T ; L^{2}(\Omega)^{d}\right), \boldsymbol{v}_{0} \in \boldsymbol{H}, \widetilde{\mu}^{p} \in L^{\infty}\left(\Omega_{p}\right)$ and there exists $\mu_{m}>0$ such that $\widetilde{\mu}^{p}(x) \geq \mu_{m}>0$, for a.e. $x \in \Omega_{p}$, $\alpha \in L^{\infty}(\Sigma)$ with $\alpha_{M}:=\|\alpha\|_{L^{\infty}(\Sigma)}$ and there exists $\alpha_{m}>0$ such that $\alpha(x) \geq \alpha_{m}>0$, for a.e. $x \in \Sigma$. The 
tensors $\boldsymbol{K}$ and $\boldsymbol{\beta}$ are supposed to be time-independent, symmetric, uniformly bounded and positive definite. Thus, there exist $k_{m}, \beta_{m}, k_{M}, \beta_{M}>0$ such that :

$$
\begin{aligned}
& \forall \xi \in \mathbb{R}^{d}, \quad k_{m}|\xi|^{2} \leq \boldsymbol{K}^{-1}(x) \xi \cdot \xi \leq k_{M}|\xi|^{2}, \quad \text { for a.e. } x \in \Omega_{p}, \\
& \forall \xi \in \mathbb{R}^{d}, \quad \beta_{m}|\xi|^{2} \leq \boldsymbol{\beta}(x) \xi \cdot \xi \leq \beta_{M}|\xi|^{2}, \quad \text { for a.e. } x \in \Sigma,
\end{aligned}
$$

where $|$.$| denotes the Euclidean norm in \mathbb{R}^{d}$.

With the condition $\llbracket \boldsymbol{v} \cdot \boldsymbol{n} \rrbracket_{\Sigma}=0$, it is clear that the mass continuity equation $\boldsymbol{\nabla} \cdot \boldsymbol{v}=0$ holds over the whole domain $\Omega$, as shown in Proposition 2.2.

Let us define the Hilbert space :

$$
\boldsymbol{W}:=\left\{\boldsymbol{u} \in L^{2}(\Omega)^{d} ; \boldsymbol{\nabla} \cdot \boldsymbol{u}=0 \text { in } \Omega ; \boldsymbol{u}^{f} \in H^{1}\left(\Omega_{f}\right)^{d}, \boldsymbol{u}^{p} \in H^{1}\left(\Omega_{p}\right)^{d} ; \llbracket \boldsymbol{u} \cdot \boldsymbol{n} \rrbracket_{\Sigma}=0 ; \boldsymbol{u}_{\mid \Gamma}^{f}=0\right\}
$$

equipped with the natural inner product and associated hilbertian norm defined by :

$$
\|\boldsymbol{u}\|_{\boldsymbol{W}}^{2}:=\|\boldsymbol{u}\|_{0, \Omega}^{2}+\left\|\nabla \boldsymbol{u}^{f}\right\|_{0, \Omega_{f}}^{2}+\left\|\nabla \boldsymbol{u}^{p}\right\|_{0, \Omega_{p}}^{2}, \quad \forall \boldsymbol{u} \in \boldsymbol{W}
$$

In this way, the first condition $\llbracket \boldsymbol{v} \cdot \boldsymbol{n} \rrbracket_{\Sigma}=0$ in Eq. (19) is included in the space of solutions $\boldsymbol{W}$ as shown in Proposition 2.2 further.

A function $\boldsymbol{u} \in \boldsymbol{W}$ also satisfies using the Stokes theorem or Green formula: $\int_{\Sigma} \boldsymbol{u} \cdot \boldsymbol{n} \mathrm{d} s=\int_{\Omega_{p}} \boldsymbol{\nabla} \cdot \boldsymbol{u} \mathrm{d} x=0$.

Let us consider the functional setting below identifying the dual space $\boldsymbol{H}^{\prime}$ to $\boldsymbol{H}$ :

$$
\boldsymbol{W} \hookrightarrow \boldsymbol{H} \simeq \boldsymbol{H}^{\prime} \hookrightarrow \boldsymbol{W}^{\prime}
$$

and observe that the continuous imbedding $\boldsymbol{W} \hookrightarrow \boldsymbol{H}$ is dense (and compact) since we have $\boldsymbol{V} \hookrightarrow \boldsymbol{W} \boldsymbol{H}$ and $\boldsymbol{V}$ is dense in $\boldsymbol{H}$, e.g. [64] or [19, Sect. IV.3.3].

Remark 2.1 (On the weak sense for the traces of stress vectors on $\Sigma$ ).

Let us take $\boldsymbol{v} \in L^{2}(0, T ; \boldsymbol{W})$ and $p \in L^{2}\left(0, T ; L^{2}(\Omega)\right)$ satisfying Eq. (18). Then we have for the stress tensor in $\Omega_{f}$ or $\Omega_{p}: \boldsymbol{\sigma}(\boldsymbol{v}, p)^{f} \in L^{2}\left(0, T ; L^{2}\left(\Omega_{f}\right)^{d \times d}\right), \boldsymbol{\sigma}(\boldsymbol{v}, p)^{p} \in L^{2}\left(0, T ; L^{2}\left(\Omega_{p}\right)^{d \times d}\right)$, such that: $\boldsymbol{\nabla} \cdot \boldsymbol{\sigma}(\boldsymbol{v}, p)^{f} \in$ $H^{-1}\left(0, T ; L^{2}\left(\Omega_{f}\right)^{d}\right)$ and $\boldsymbol{\nabla} \cdot \boldsymbol{\sigma}(\boldsymbol{v}, p)^{p} \in H^{-1}\left(0, T ; L^{2}\left(\Omega_{p}\right)^{d}\right)$. Since $\Sigma$ is a closed surface, the normal trace theorem in $\boldsymbol{H}_{\text {div }}\left(\Omega_{f}\right)$ or $\boldsymbol{H}_{\text {div }}\left(\Omega_{p}\right)$, e.g. [19,35], ensures to define in a weak sense the traces $\boldsymbol{\sigma}(\boldsymbol{v}, p)^{f} \cdot \boldsymbol{n}_{\mid \Sigma} \in$ $H^{-1}\left(0, T ; H^{-1 / 2}(\Sigma)^{d}\right)$ and $\boldsymbol{\sigma}(\boldsymbol{v}, p)^{p} \cdot \boldsymbol{n}_{\mid \Sigma} \in H^{-1}\left(0, T ; H^{-1 / 2}(\Sigma)^{d}\right)$ on both sides of $\Sigma$.

So, this ensures that $\overline{\boldsymbol{\sigma}(\boldsymbol{v}, p) \cdot \boldsymbol{n}_{\Sigma}} \in H^{-1}\left(0, T ; H^{-1 / 2}(\Sigma)^{d}\right)$ and $\llbracket \boldsymbol{\sigma}(\boldsymbol{v}, p) \cdot \boldsymbol{n} \rrbracket_{\Sigma} \in H^{-1}\left(0, T ; H^{-1 / 2}(\Sigma)^{d}\right)$.

More precisely if $(\boldsymbol{v}, p)$ also verifies Eq. (19), since $\llbracket \boldsymbol{v} \rrbracket_{\Sigma}$ belongs to $L^{2}\left(0, T ; H^{1 / 2}(\Sigma)^{d}\right)$ and $\alpha$ is a bounded function on $\Sigma$, the second equation in (19) shows that $\overline{\boldsymbol{\sigma}(\boldsymbol{v}, p) \cdot \boldsymbol{n}_{\Sigma}} \cdot \boldsymbol{\tau}_{j} \in L^{2}\left(0, T ; L^{2}(\Sigma)\right)$, for $1 \leq j \leq d-1$, and thus also ${\overline{\widetilde{\mu} \nabla \boldsymbol{v} \cdot \boldsymbol{n}_{\Sigma}}}_{\boldsymbol{\tau}} \boldsymbol{\tau}_{j} \in L^{2}\left(0, T ; L^{2}(\Sigma)\right)$.

Similarly, since $\overline{\boldsymbol{v}}_{\Sigma}$ belongs to $L^{2}\left(0, T ; H^{1 / 2}(\Sigma)^{d}\right)$, the last equation in (19) with $\boldsymbol{\beta}$ bounded on $\Sigma$ shows that $\llbracket \boldsymbol{\sigma}(\boldsymbol{v}, p) \cdot \boldsymbol{n} \rrbracket_{\Sigma} \in L^{2}\left(0, T ; L^{2}(\Sigma)^{d}\right)$.

Thus, with usual Green formula from Eq. (18), the integral terms on $\Gamma$ vanishing with the homogeneous Dirichlet boundary condition, we have for all $\varphi \in W$ :

$$
\begin{aligned}
\rho\left\langle\partial_{t} \boldsymbol{v}, \boldsymbol{\varphi}\right\rangle_{\boldsymbol{W}^{\prime}, \boldsymbol{W}}+\mu \int_{\Omega_{f}} \nabla \boldsymbol{v}: \nabla \boldsymbol{\varphi} \mathrm{d} x & +\int_{\Omega_{p}} \widetilde{\mu}^{p} \nabla \boldsymbol{v}: \nabla \boldsymbol{\varphi} \mathrm{d} x+\mu \int_{\Omega_{p}}\left(\boldsymbol{K}^{-1} \boldsymbol{v}\right) \cdot \boldsymbol{\varphi} \mathrm{d} x \\
& +\left\langle\boldsymbol{\sigma}(\boldsymbol{v}, p)^{f} \cdot \boldsymbol{n}, \boldsymbol{\varphi}^{f}\right\rangle_{-1 / 2, \Sigma}-\left\langle\boldsymbol{\sigma}(\boldsymbol{v}, p)^{p} \cdot \boldsymbol{n}, \boldsymbol{\varphi}^{p}\right\rangle_{-1 / 2, \Sigma}=\rho \int_{\Omega} \boldsymbol{f} \cdot \boldsymbol{\varphi} \mathrm{d} x
\end{aligned}
$$


Now following [7], we use the key equality that holds for any bilinear form :

$$
\left\langle\boldsymbol{\sigma}(\boldsymbol{v}, p)^{f} \cdot \boldsymbol{n}, \boldsymbol{\varphi}^{f}\right\rangle_{-1 / 2, \Sigma}-\left\langle\boldsymbol{\sigma}(\boldsymbol{v}, p)^{p} \cdot \boldsymbol{n}, \boldsymbol{\varphi}^{p}\right\rangle_{-1 / 2, \Sigma}=\left\langle\overline{\boldsymbol{\sigma}(\boldsymbol{v}, p) \cdot \boldsymbol{n}_{\Sigma}}, \llbracket \boldsymbol{\varphi} \rrbracket_{\Sigma}\right\rangle_{-1 / 2, \Sigma}+\left\langle\llbracket \boldsymbol{\sigma}(\boldsymbol{v}, p) \cdot \boldsymbol{n} \rrbracket_{\Sigma}, \overline{\boldsymbol{\varphi}}_{\Sigma}\right\rangle_{-1 / 2, \Sigma}
$$

This simple equality justifies the idea of looking for general jump interface conditions as equations giving both the stress vector jump $\llbracket \boldsymbol{\sigma}(\boldsymbol{v}, p) \cdot \boldsymbol{n} \rrbracket_{\Sigma}$ and the stress vector mean ${\overline{\boldsymbol{\sigma}}(\boldsymbol{v}, p) \cdot \boldsymbol{n}_{\Sigma}}_{\mathrm{s}}$ on as early noticed in $[5,6]$ for scalar elliptic problems. Therefore, Eq. (18) admits the general weak form below :

$$
\begin{aligned}
\rho\left\langle\partial_{t} \boldsymbol{v}, \boldsymbol{\varphi}\right\rangle_{\boldsymbol{W}^{\prime}, \boldsymbol{W}} & +\mu \int_{\Omega_{f}} \nabla \boldsymbol{v}: \nabla \boldsymbol{\varphi} \mathrm{d} x+\int_{\Omega_{p}} \widetilde{\mu}^{p} \nabla \boldsymbol{v}: \nabla \boldsymbol{\varphi} \mathrm{d} x+\mu \int_{\Omega_{p}}\left(\boldsymbol{K}^{-1} \boldsymbol{v}\right) \cdot \boldsymbol{\varphi} \mathrm{d} x \\
& +\left\langle{\overline{\boldsymbol{\sigma}(\boldsymbol{v}, p) \cdot \boldsymbol{n}_{\Sigma}}}_{\llbracket} \llbracket \boldsymbol{\varphi} \rrbracket_{\Sigma}\right\rangle_{-1 / 2, \Sigma}+\left\langle\llbracket \boldsymbol{\sigma}(\boldsymbol{v}, p) \cdot \boldsymbol{n} \rrbracket_{\Sigma}, \overline{\boldsymbol{\varphi}}_{\Sigma}\right\rangle_{-1 / 2, \Sigma}=\rho \int_{\Omega} \boldsymbol{f} \cdot \boldsymbol{\varphi} \mathrm{d} x, \quad \forall \boldsymbol{\varphi} \in \boldsymbol{W} .
\end{aligned}
$$

Then, using the interface conditions (19) with $\llbracket \boldsymbol{\varphi} \cdot \boldsymbol{n} \rrbracket_{\Sigma}=0$ to deal with the second interface condition, we get the weak form for all $\varphi \in W$ :

$$
\begin{aligned}
\rho\left\langle\partial_{t} \boldsymbol{v}, \boldsymbol{\varphi}\right\rangle_{\boldsymbol{W}^{\prime}, \boldsymbol{W}} & +\mu \int_{\Omega_{f}} \nabla \boldsymbol{v}: \nabla \boldsymbol{\varphi} \mathrm{d} x+\int_{\Omega_{p}} \widetilde{\mu}^{p} \nabla \boldsymbol{v}: \nabla \boldsymbol{\varphi} \mathrm{d} x+\mu \int_{\Omega_{p}}\left(\boldsymbol{K}^{-1} \boldsymbol{v}\right) \cdot \boldsymbol{\varphi} \mathrm{d} x \\
& +\frac{\mu}{\sqrt{K}} \sum_{j=1}^{d-1} \int_{\Sigma} \alpha \llbracket \boldsymbol{v} \cdot \boldsymbol{\tau}_{j} \rrbracket_{\Sigma} \llbracket \boldsymbol{\varphi} \cdot \boldsymbol{\tau}_{j} \rrbracket_{\Sigma} \mathrm{d} s+\frac{\mu}{\sqrt{K}} \int_{\Sigma}\left(\boldsymbol{\beta} \overline{\boldsymbol{v}}_{\Sigma}\right) \cdot \overline{\boldsymbol{\varphi}}_{\Sigma} \mathrm{d} s=\rho \int_{\Omega} \boldsymbol{f} \cdot \boldsymbol{\varphi} \mathrm{d} x, \quad \forall \boldsymbol{\varphi} \in \boldsymbol{W} .
\end{aligned}
$$

Since $\llbracket \boldsymbol{\varphi} \cdot \boldsymbol{n} \rrbracket_{\Sigma}=0$ for all $\boldsymbol{\varphi} \in \boldsymbol{W}$, the fifth term also reads :

$$
\frac{\mu}{\sqrt{K}} \sum_{j=1}^{d-1} \int_{\Sigma} \alpha \llbracket \boldsymbol{v} \cdot \boldsymbol{\tau}_{j} \rrbracket_{\Sigma} \llbracket \boldsymbol{\varphi} \cdot \boldsymbol{\tau}_{j} \rrbracket_{\Sigma} \mathrm{d} s=\frac{\mu}{\sqrt{K}} \int_{\Sigma} \alpha \llbracket \boldsymbol{v} \rrbracket_{\Sigma} \cdot \llbracket \boldsymbol{\varphi} \rrbracket_{\Sigma} \mathrm{d} s .
$$

Therefore, we propose the following weak formulation of Leray's type for the Stokes/Brinkman problem (18-19) with $\boldsymbol{f} \in L^{2}\left(0, T ; L^{2}(\Omega)^{d}\right)$ using divergence-free test functions in $\boldsymbol{W}$.

Find $\boldsymbol{v} \in \mathcal{C}([0, T] ; \boldsymbol{H}) \cap L^{2}(0, T ; \boldsymbol{W})$ with $\boldsymbol{v}^{\prime}:=\frac{d \boldsymbol{v}}{d t} \in L^{2}\left(0, T ; \boldsymbol{W}^{\prime}\right)$ such that $\boldsymbol{v}(t=0)=\boldsymbol{v}_{0}$ in $\boldsymbol{H}$ and satisfying for all $\varphi \in W$ :

$$
\begin{aligned}
\rho\left\langle\frac{d \boldsymbol{v}}{d t}, \boldsymbol{\varphi}\right\rangle_{\boldsymbol{W}^{\prime}, \boldsymbol{W}}+\mu \int_{\Omega_{f}} \nabla \boldsymbol{v}: \nabla \boldsymbol{\varphi} \mathrm{d} x & +\int_{\Omega_{p}} \widetilde{\mu}^{p} \nabla \boldsymbol{v}: \nabla \boldsymbol{\varphi} \mathrm{d} x+\mu \int_{\Omega_{p}}\left(\boldsymbol{K}^{-1} \boldsymbol{v}\right) \cdot \boldsymbol{\varphi} \mathrm{d} x \\
& +\frac{\mu}{\sqrt{K}} \int_{\Sigma} \alpha \llbracket \boldsymbol{v} \rrbracket_{\Sigma} \cdot \llbracket \boldsymbol{\varphi} \rrbracket_{\Sigma} \mathrm{d} s+\frac{\mu}{\sqrt{K}} \int_{\Sigma}\left(\boldsymbol{\beta} \overline{\boldsymbol{v}}_{\Sigma}\right) \cdot \overline{\boldsymbol{\varphi}}_{\Sigma} \mathrm{d} s=\rho \int_{\Omega} \boldsymbol{f} \cdot \boldsymbol{\varphi} \mathrm{d} x \\
& \text { for a.e. } t \in] 0, T[\quad \forall \boldsymbol{\varphi} \in \boldsymbol{W} .
\end{aligned}
$$

\subsection{Preliminary results}

Proposition 2.2 (Normal velocity jump on $\Sigma$ ).

Let us consider the configuration shown in Figure 1.

Let $\boldsymbol{v} \in L^{2}(\Omega)^{d}$ such that $\boldsymbol{\nabla} \cdot \boldsymbol{v}^{f}=0$ in $\Omega_{f}$ and $\boldsymbol{\nabla} \cdot \boldsymbol{v}^{p}=0$ in $\Omega_{p}$. Then $\boldsymbol{\nabla} \cdot \boldsymbol{v}=0$ over the whole domain $\Omega$ if and only if $\llbracket \boldsymbol{v} \cdot \boldsymbol{n} \rrbracket_{\Sigma}:=\left(\boldsymbol{v}^{f} \cdot \boldsymbol{n}_{\mid \Sigma}-\boldsymbol{v}^{p} \cdot \boldsymbol{n}_{\mid \Sigma}\right)=0$ on $\Sigma$.

Proof. By the normal trace theorem [35, Theorem 2.5] in $\boldsymbol{H}_{\text {div }}\left(\Omega_{f}\right)$ or $\boldsymbol{H}_{\text {div }}\left(\Omega_{p}\right) \boldsymbol{v}^{f}$ and $\boldsymbol{v}^{p}$ admit a normal trace on $\Sigma$ in $H^{-1 / 2}(\Sigma): \boldsymbol{v}^{f} \cdot \boldsymbol{n}_{\mid \Sigma}$ and $\boldsymbol{v}^{p} \cdot \boldsymbol{n}_{\mid \Sigma}$, respectively. Since $\Sigma$ is a closed surface in $\mathbb{R}^{d-1}, \boldsymbol{v}^{f} \cdot \boldsymbol{n}_{\mid \Sigma}$ from the 
side of $\Omega_{f}$ is defined as the restriction of the linear and continuous form $\boldsymbol{v}^{f} \cdot \boldsymbol{n}_{\mid \partial \Omega_{f}} \in H^{-1 / 2}\left(\partial \Omega_{f}\right)$ to functions in $H^{1 / 2}(\Sigma)$; see [40].

Then, for all $\phi \in H_{0}^{1}(\Omega)$, we have with Green's formula :

$$
\begin{aligned}
\langle\nabla \cdot \boldsymbol{v}, \phi\rangle_{-1, \Omega} & =-\int_{\Omega} \boldsymbol{v} \cdot \nabla \phi \mathrm{d} x=-\int_{\Omega_{f}} \boldsymbol{v}^{f} \cdot \nabla \phi \mathrm{d} x-\int_{\Omega_{p}} \boldsymbol{v}^{p} \cdot \nabla \phi \mathrm{d} x \\
& =\left\langle\boldsymbol{v}^{f} \cdot \boldsymbol{n}_{\mid \Sigma}, \phi_{\mid \Sigma}\right\rangle_{-1 / 2, \Sigma}-\left\langle\boldsymbol{v}^{p} \cdot \boldsymbol{n}_{\mid \Sigma}, \phi_{\mid \Sigma}\right\rangle_{-1 / 2, \Sigma} \\
& =\left\langle\llbracket \boldsymbol{v} \cdot \boldsymbol{n} \rrbracket_{\Sigma}, \phi_{\mid \Sigma}\right\rangle_{-1 / 2, \Sigma} .
\end{aligned}
$$

Now, let us observe that any function $\psi \in H^{1 / 2}(\Sigma)$ can be extended in $\Omega$ by a function $\phi \in H_{0}^{1}(\Omega)$. It suffices to take the solution $\phi \in H_{0}^{1}(\Omega)$ of the Dirichlet problem: $\Delta \phi=0$ in $\Omega_{f} \cup \Omega_{p}$ with $\phi_{\mid \Gamma}=0$ and $\phi_{\mid \Sigma}=\psi$.

Then, the previous equality gives the desired result.

Let us recall the classical solvability result for a linear abstract parabolic problem [50].

Theorem 2.3 (J.-L. Lions' theorem for an abstract parabolic problem).

Let $H$ and $V \subset H$ be two Hilbert spaces such that the continuous imbedding $V \hookrightarrow H$ is dense and consider, by identifying $H$ and its dual space $H^{\prime}$, the functional setting:

$$
V \hookrightarrow H \simeq H^{\prime} \hookrightarrow V^{\prime}
$$

with $(., .)_{H}$ denoting the inner product in $H$ and $\|\cdot\|_{H}$ the associated hilbertian norm.

For a.e. $t \in[0, T]$, let $a(t ; .,):. V \times V \rightarrow \mathbb{R}$ be a bilinear continuous and coercive form such that the function $t \mapsto a(t ; v, w)$ is mesurable for all $v, w \in V$ and thus:

i) $\quad|a(t ; v, w)| \leq M\|v\|_{V}\|w\|_{V}, \quad$ for a.e. $t \in[0, T], \quad$ for all $v, w \in V$,

ii) $|a(t ; v, v)| \geq \alpha\|v\|_{V}^{2}-\lambda\|v\|_{H}^{2}$, for a.e. $t \in[0, T], \quad$ for all $v \in V$,

where $\alpha>0, \lambda$ and $M$ are constants.

Then, for all $f \in L^{2}\left(0, T ; V^{\prime}\right)$ and $u_{0} \in H$, there exists a unique solution $u \in \mathcal{C}([0, T] ; H) \cap L^{2}(0, T ; V)$ with $u^{\prime} \in L^{2}\left(0, T ; V^{\prime}\right)$ verifying the weak problem

$$
\left\{\begin{aligned}
\left\langle\frac{d u}{d t}(t), v\right\rangle_{V^{\prime}, V}+a(t ; u(t), v) & =\langle f(t), v\rangle_{V^{\prime}, V} \quad \text { for a.e. } t \in[0, T], \quad \text { for all } v \in V, \\
u(t=0) & =u_{0} .
\end{aligned}\right.
$$

Proof. See [50, Chap. 3.4] or [31].

This can be proved using Nečas' generalization [53] of Lax-Milgram theorem when the solution space is different from the space of test functions, which is a consequence of the Open Mapping theorem and Closed Range theorem, both due to Banach [20, Sect. II.3].

In the proof of the theorem further, we shall also need the auxiliary result below.

Lemma 2.4 (Divergence-free extension for $H^{1 / 2}(\Gamma)^{d}$ ).

Let $\Omega \subset \mathbb{R}^{d}$ be a bounded Lipschitz domain, let $\Gamma:=\partial \Omega$ be its boundary and $\boldsymbol{n}$ the outward unit normal vector to $\Gamma$. Then for any $\boldsymbol{\psi} \in H^{1 / 2}(\Gamma)^{d}$, there exists a function $\boldsymbol{u} \in H^{1}(\Omega)^{d}$ such that $\boldsymbol{\nabla} \cdot \boldsymbol{u}=0$ in $\Omega$ and

$$
\boldsymbol{\psi}=\boldsymbol{u}_{\mid \Gamma}+\frac{1}{|\Gamma|}\left(\int_{\Gamma} \boldsymbol{\psi} \cdot \boldsymbol{n} \mathrm{d} s\right) \boldsymbol{n}, \quad \text { where }|\Gamma| \text { is the }(d-1) \text { measure of } \Gamma \text {. }
$$


Proof. Since the boundary $\Gamma$ is Lipschitz continuous, the unit normal vector $\boldsymbol{n}$ on $\Gamma$ belongs to $H^{1 / 2}(\Gamma)^{d}[40]$ and verifies

$$
\int_{\Gamma} \boldsymbol{n} \cdot \boldsymbol{n} \mathrm{d} s=\int_{\Gamma}|\boldsymbol{n}|^{2} \mathrm{~d} s=|\Gamma| .
$$

Now for any $\boldsymbol{\psi} \in H^{1 / 2}(\Gamma)^{d}$, let us define $\widetilde{\boldsymbol{\psi}} \in H^{1 / 2}(\Gamma)^{d}$ by

$$
\widetilde{\boldsymbol{\psi}}:=\boldsymbol{\psi}-\frac{1}{|\Gamma|}\left(\int_{\Gamma} \boldsymbol{\psi} \cdot \boldsymbol{n} \mathrm{d} s\right) \boldsymbol{n}, \quad \text { such that } \quad \int_{\Gamma} \widetilde{\boldsymbol{\psi}} \cdot \boldsymbol{n} \mathrm{d} s=0
$$

Then following [19, Proposition IV.5.2], there exists a solution $(\boldsymbol{u}, p) \in H^{1}(\Omega)^{d} \times L_{0}^{2}(\Omega)$ to the Stokes problem with non-homogeneous Dirichlet boundary condition :

$$
\left\{\begin{aligned}
-\Delta \boldsymbol{u}+\nabla p & =0, & & \text { in } \Omega, \\
\boldsymbol{\nabla} \cdot \boldsymbol{u} & =0, & & \text { in } \Omega, \\
\boldsymbol{u}_{\mid \Gamma} & =\widetilde{\boldsymbol{\psi}}, & & \text { on } \Gamma .
\end{aligned}\right.
$$

This shows the desired result.

\subsection{Main result of solvability for the Stokes/Brinkman problem}

Then, we prove the following main result of solvability in this section.

Theorem 2.5 (Global well-posedness in time of the Stokes/Brinkman problem).

For any $\boldsymbol{v}_{0} \in \boldsymbol{W}$ in addition to the basic assumptions on the data $\boldsymbol{f}, \widetilde{\mu}^{p}, \boldsymbol{K}, \alpha, \boldsymbol{\beta}$, there exists a unique solution $(\boldsymbol{v}, p)$ to the problem (18-19) such that $\boldsymbol{v} \in \mathcal{C}([0, T] ; \boldsymbol{H}) \cap L^{2}(0, T ; \boldsymbol{W})$ with $\boldsymbol{v}^{\prime} \in L^{2}(0, T ; \boldsymbol{H})$ and $p \in L^{2}\left(0, T ; L^{2}(\Omega)\right)$, which satisfies the weak form (22) and the energy equality below:

$$
\begin{aligned}
\frac{\rho}{2}\|\boldsymbol{v}(t)\|_{0, \Omega}^{2}+\mu \int_{0}^{t} \int_{\Omega_{f}}|\nabla \boldsymbol{v}|^{2} \mathrm{~d} x \mathrm{~d} \tau & +\int_{0}^{t} \int_{\Omega_{p}} \widetilde{\mu}^{p}|\nabla \boldsymbol{v}|^{2} \mathrm{~d} x \mathrm{~d} \tau+\mu \int_{0}^{t} \int_{\Omega_{p}}\left(\boldsymbol{K}^{-1} \boldsymbol{v}\right) \cdot \boldsymbol{v} \mathrm{d} x \mathrm{~d} \tau \\
& +\frac{\mu}{\sqrt{K}} \int_{0}^{t} \int_{\Sigma} \alpha \llbracket \boldsymbol{v} \rrbracket_{\Sigma} \cdot \llbracket \boldsymbol{v} \rrbracket_{\Sigma} \mathrm{d} s \mathrm{~d} \tau+\frac{\mu}{\sqrt{K}} \int_{0}^{t} \int_{\Sigma}\left(\boldsymbol{\beta} \overline{\boldsymbol{v}}_{\Sigma}\right) \cdot \overline{\boldsymbol{v}}_{\Sigma} \mathrm{d} s \mathrm{~d} \tau \\
& =\frac{\rho}{2}\left\|\boldsymbol{v}_{0}\right\|_{0, \Omega}^{2}+\rho \int_{0}^{t} \int_{\Omega} \boldsymbol{f} \cdot \boldsymbol{v} \mathrm{d} x \mathrm{~d} \tau, \quad \text { for all } t \in[0, T] .
\end{aligned}
$$

Moreover, there exists a function $q \in L^{2}\left(0, T ; L^{2}(\Omega)\right)$ with each restriction $q^{f} \in L^{2}\left(0, T ; L_{0}^{2}\left(\Omega_{f}\right)\right)$ and $q^{p} \in$ $L^{2}\left(0, T ; L_{0}^{2}\left(\Omega_{p}\right)\right)$ both defined uniquely up to an additive constant, such that the pressure field $p \in L^{2}\left(0, T ; L^{2}(\Omega)\right)$ is defined uniquely by:

$$
p_{\mid \Omega_{f}}:=q^{f}+\frac{\Pi_{0}}{2}, \quad p_{\mid \Omega_{p}}:=q^{p}-\frac{\Pi_{0}}{2}, \quad \text { such that } \llbracket p-q \rrbracket_{\Sigma}=\Pi_{0},
$$

where the constant quantity $\Pi_{0}$ is defined from $(\boldsymbol{v}, q)$ by :

$$
\Pi_{0}:=\frac{1}{|\Sigma|}\left\langle\llbracket \boldsymbol{\sigma}(\boldsymbol{v}, q) \cdot \boldsymbol{n} \rrbracket_{\Sigma}-\frac{\mu}{\sqrt{K}} \boldsymbol{\beta} \overline{\boldsymbol{v}}_{\Sigma}, \boldsymbol{n}\right\rangle_{-1 / 2, \Sigma} .
$$

Proof.

a) Existence and uniqueness of the weak solution $v$ to (22). 
First, it is an easy matter to verify that the assumptions of Lions' Theorem 2.3 for abstract parabolic problems are satisfied with $\boldsymbol{v}_{0}$ only in $\boldsymbol{H}$. Indeed, Eq. (22) takes the general form :

$$
\left\langle\frac{d \boldsymbol{v}}{d t}(t), \boldsymbol{w}\right\rangle_{\boldsymbol{W}^{\prime}, \boldsymbol{W}}+a(\boldsymbol{v}(t), \boldsymbol{w})=(\boldsymbol{f}(t), \boldsymbol{w})_{\boldsymbol{H}} \quad \text { for a.e. } t \in[0, T], \quad \text { for all } \boldsymbol{w} \in \boldsymbol{W} .
$$

The required continuity and coercivity over $\boldsymbol{W}$ of the bilinear form $a(.,$.$) corresponding to the elliptic operator in$ (22) are obtained with the basic assumptions on the data through standard calculations using Cauchy-Schwarz inequality, Poincaré inequality in $H_{0 \Gamma}^{1}\left(\Omega_{f}\right)^{d}$, and classical trace inequalities on $\Sigma$ for functions in $H^{1}\left(\Omega_{f}\right)^{d}$ or $H^{1}\left(\Omega_{p}\right)^{d}$.

This ensures the existence and uniqueness of the solution $\boldsymbol{v}$ to the weak problem (22).

Since the continuous imbedding $\boldsymbol{W} \hookrightarrow \boldsymbol{H}$ is dense with the identification of $\boldsymbol{H}$ and $\boldsymbol{H}^{\prime}$, with $\boldsymbol{v} \in L^{2}(0, T, \boldsymbol{W})$ with $\boldsymbol{v}^{\prime} \in L^{2}\left(0, T, \boldsymbol{W}^{\prime}\right)$, a classical application of Lions-Magenes theorem [50] ensures that $\boldsymbol{v} \in \mathcal{C}([0, T] ; \boldsymbol{H})$ and

$$
\left\langle\frac{d \boldsymbol{v}}{d t}(t), \boldsymbol{v}(t)\right\rangle_{\boldsymbol{W}^{\prime}, \boldsymbol{W}}=\frac{1}{2} \frac{d}{d t}((\boldsymbol{v}(t), \boldsymbol{v}(t)))_{\boldsymbol{H}}=\frac{1}{2} \frac{d}{d t}\|\boldsymbol{v}(t)\|_{\boldsymbol{H}}^{2}, \quad \text { a.e. } t \in[0, T]
$$

Since $\boldsymbol{f} \in L^{2}\left(0, T ; L^{2}(\Omega)^{d}\right)$, let us show that $\boldsymbol{v}^{\prime} \in L^{2}(0, T ; \boldsymbol{H})$ with the initial condition $\boldsymbol{v}_{0} \in \boldsymbol{W}$. By choosing in (22) the test function $\boldsymbol{\varphi}=2 \boldsymbol{v}^{\prime}(t) \in \boldsymbol{W}$ for a.e. $\left.t \in\right] 0, T[$, we have :

$$
\begin{aligned}
2 \rho\left\|\frac{d \boldsymbol{v}}{d t}(t)\right\|_{0, \Omega}^{2}+\mu \frac{d}{d t} \int_{\Omega_{f}}|\nabla \boldsymbol{v}(t)|^{2} \mathrm{~d} x & +\frac{d}{d t} \int_{\Omega_{p}} \widetilde{\mu}^{p}|\nabla \boldsymbol{v}(t)|^{2} \mathrm{~d} x+\mu \frac{d}{d t} \int_{\Omega_{p}}\left(\boldsymbol{K}^{-1} \boldsymbol{v}(t)\right) \cdot \boldsymbol{v}(t) \mathrm{d} x \\
& +\frac{\mu}{\sqrt{K}} \frac{d}{d t} \int_{\Sigma} \alpha \llbracket \boldsymbol{v}(t) \rrbracket_{\Sigma} \cdot \llbracket \boldsymbol{v}(t) \rrbracket_{\Sigma} \mathrm{d} s+\frac{\mu}{\sqrt{K}} \frac{d}{d t} \int_{\Sigma}\left(\boldsymbol{\beta}{\overline{\boldsymbol{v}(t)_{\Sigma}}}_{\overline{2}} \cdot \overline{\boldsymbol{v}(t)_{\Sigma}} \mathrm{d} s\right. \\
& =2 \rho \int_{\Omega} \boldsymbol{f}(t) \cdot \frac{d \boldsymbol{v}}{d t}(t) \mathrm{d} x
\end{aligned}
$$

The right-hand side term is bounded as below using the Cauchy-Schwarz inequality and the inequality $2 a b \leq$ $a^{2}+b^{2}$ for all $a, b \in \mathbb{R}$ :

$$
2 \rho\left|\int_{\Omega} \boldsymbol{f}(t) \cdot \frac{d \boldsymbol{v}}{d t}(t) \mathrm{d} x\right| \leq \rho\left\|\frac{d \boldsymbol{v}}{d t}(t)\right\|_{0, \Omega}^{2}+\rho\|\boldsymbol{f}(t)\|_{0, \Omega}^{2}
$$

Using this bound and integrating in time over $(0, t)$ for a.e. $t \in[0, T]$, it yields with the initial condition :

$$
\begin{aligned}
& \rho \int_{0}^{t}\left\|\frac{d \boldsymbol{v}}{d t}(\tau)\right\|_{0, \Omega}^{2} \mathrm{~d} \tau+\mu \int_{\Omega_{f}}|\nabla \boldsymbol{v}(t)|^{2} \mathrm{~d} x+\int_{\Omega_{p}} \widetilde{\mu}^{p}|\nabla \boldsymbol{v}(t)|^{2} \mathrm{~d} x+\mu \int_{\Omega_{p}}\left(\boldsymbol{K}^{-1} \boldsymbol{v}(t)\right) \cdot \boldsymbol{v}(t) \mathrm{d} x \\
&+\frac{\mu}{\sqrt{K}} \int_{\Sigma} \alpha \llbracket \boldsymbol{v}(t) \rrbracket_{\Sigma} \cdot \llbracket \boldsymbol{v}(t) \rrbracket_{\Sigma} \mathrm{d} s+\frac{\mu}{\sqrt{K}} \int_{\Sigma}\left(\boldsymbol{\beta} \overline{\boldsymbol{v}(t)_{\Sigma}}\right) \cdot \overline{\boldsymbol{v}(t)_{\Sigma}} \mathrm{d} s \\
& \leq \mu\left\|\nabla \boldsymbol{v}_{0}\right\|_{0, \Omega_{f}}^{2}+\left\|\widetilde{\mu}^{p}\right\|_{L^{\infty}\left(\Omega_{p}\right)}\left\|\nabla \boldsymbol{v}_{0}\right\|_{0, \Omega_{p}}^{2}+\mu k_{M}\left\|\boldsymbol{v}_{0}\right\|_{0, \Omega_{p}}^{2} \\
&+\frac{\mu \alpha_{M}}{\sqrt{K}}\left\|\llbracket \boldsymbol{v}_{0} \rrbracket_{\Sigma}\right\|_{0, \Sigma}^{2}+\frac{\mu \beta_{M}}{\sqrt{K}}\left\|\overline{\boldsymbol{v}_{0}}\right\|_{0, \Sigma}^{2}+\rho \int_{0}^{t}\|\boldsymbol{f}(\tau)\|_{0, \Omega}^{2} \mathrm{~d} \tau, \\
& \leq C\left(\Omega, \Omega_{f}, \Omega_{p}, T, \rho, \mu, \widetilde{\mu}^{p}, \boldsymbol{K}, \alpha, \boldsymbol{\beta}, \boldsymbol{v}_{0}, \boldsymbol{f}\right) .
\end{aligned}
$$

Then, the energy equality (23) is easily obtained by taking in (22) the test function $\boldsymbol{\varphi}=\boldsymbol{v}(t) \in \boldsymbol{W}$ and integrating in time over $(0, t)$ for all $t \in[0, T]$ with the initial condition. 


\section{b) Interpretation of weak problem (22) and recovering the momentum equations.}

In the last step of the proof, the weak velocity solution $\boldsymbol{v}$ must be shown to satisfy in some sense the momentum equations in (18) and the jump interface conditions Eq. (19). It requires to recover the pressure field $p$ by subdomains, either in $\Omega_{f}$ or in $\Omega_{p}$, as made in $[7,8]$ for the jump embedded boundary conditions similar to the present case. Another possibility is to use a mixed weak formulation with the Babuška-Brezzi inf-sup theory, e.g. [35]; see also [36,37] for related transmission problems.

By taking any $\varphi \in \boldsymbol{W}$ in $(22)$ such that $\varphi^{p} \in H_{0}^{1}\left(\Omega_{p}\right)^{d}$ and $\varphi^{f}=0$, we have for a.e. $\left.t \in\right] 0, T[$ :

$$
\rho \int_{\Omega_{p}} \frac{d \boldsymbol{v}}{d t} \cdot \boldsymbol{\varphi} \mathrm{d} x+\int_{\Omega_{p}} \widetilde{\mu}^{p} \nabla \boldsymbol{v}: \nabla \boldsymbol{\varphi} \mathrm{d} x+\mu \int_{\Omega_{p}}\left(\boldsymbol{K}^{-1} \boldsymbol{v}\right) \cdot \boldsymbol{\varphi} \mathrm{d} x=\rho \int_{\Omega_{p}} \boldsymbol{f} \cdot \boldsymbol{\varphi} \mathrm{d} x
$$

which gives by integration by parts :

$$
\rho \int_{\Omega_{p}} \frac{d \boldsymbol{v}}{d t} \cdot \boldsymbol{\varphi} \mathrm{d} x-\int_{\Omega_{p}} \boldsymbol{\nabla} \cdot\left(\widetilde{\mu}^{p} \nabla \boldsymbol{v}\right) \cdot \boldsymbol{\varphi} \mathrm{d} x+\mu \int_{\Omega_{p}}\left(\boldsymbol{K}^{-1} \boldsymbol{v}\right) \cdot \boldsymbol{\varphi} \mathrm{d} x=\rho \int_{\Omega_{p}} \boldsymbol{f} \cdot \boldsymbol{\varphi} \mathrm{d} x
$$

Applying De Rham's theorem $[19,64]$ in $\Omega_{p}$ since $\boldsymbol{\nabla} \cdot \boldsymbol{\varphi}=0$, there exists $q^{p}(t) \in L_{0}^{2}\left(\Omega_{p}\right)$ which is unique since the domain $\Omega_{p}$ is connected, such that the equation below holds at least in the sense $H^{-1}\left(\Omega_{p}\right)^{d}$ for a.e. $\left.t \in\right] 0, T[$ :

$$
\rho \frac{\partial \boldsymbol{v}}{\partial t}-\nabla \cdot\left(\widetilde{\mu}^{p} \nabla \boldsymbol{v}\right)+\mu \boldsymbol{K}^{-1} \boldsymbol{v}+\nabla q^{p}=\rho \boldsymbol{f}, \quad \text { in }(0, T) \times \Omega_{p}
$$

Thus, the Brinkman equation is satisfied in $(0, T) \times \Omega_{p}$.

Doing similarly by taking any $\varphi \in \boldsymbol{W}$ in $(22)$ such that $\varphi^{f} \in H_{0}^{1}\left(\Omega_{f}\right)^{d}$ and $\varphi^{p}=0$, there exists $q^{f}(t) \in L_{0}^{2}\left(\Omega_{f}\right)$ which is unique since $\Omega_{f}$ is connected, such that the equation below holds at least in the sense $H^{-1}\left(\Omega_{f}\right)^{d}$ for a.e. $t \in] 0, T[$ :

$$
\rho \frac{\partial \boldsymbol{v}}{\partial t}-\mu \Delta \boldsymbol{v}+\nabla q^{f}=\rho \boldsymbol{f}, \quad \text { in }(0, T) \times \Omega_{f},
$$

and the Stokes equation is also verified in $(0, T) \times \Omega_{f}$. This defines the pressure field $q \in L^{2}\left(0, T ; L_{0}^{2}(\Omega)\right)$ by its restrictions $q^{f}$ over $\Omega_{f}$ and $q^{p}$ over $\Omega_{p}$, each defined uniquely up to an additive constant, and shows that $(\boldsymbol{v}, q)$ satisfies Eq. (18).

\section{c) Recovering the jump interface conditions and adjusting the pressure field $p$.}

Let us now look for the jump interface conditions on $\Sigma$ following the ideas from [7]. From the definition of the space $\boldsymbol{W}$, we have already $\llbracket \boldsymbol{v} \cdot \boldsymbol{n} \rrbracket_{\Sigma}=0$.

First, we choose any test function $\varphi \in \boldsymbol{W}$ such that $\bar{\varphi}_{\Sigma} \cdot \boldsymbol{\tau}_{j}=0$, i.e. $\boldsymbol{\varphi}_{\mid \Sigma}^{f} \cdot \boldsymbol{\tau}_{j}=-\boldsymbol{\varphi}_{\mid \Sigma}^{p} \cdot \boldsymbol{\tau}_{j}$, for $1 \leq j \leq d-1$, and thus $\llbracket \varphi \rrbracket_{\Sigma} \cdot \boldsymbol{\tau}_{j}=-2 \varphi_{\mid \Sigma}^{p} \cdot \boldsymbol{\tau}_{j}$ and $\llbracket \boldsymbol{\varphi} \cdot \boldsymbol{n} \rrbracket_{\Sigma}=0$. From one side, the weak form (22), we have for a.e. $\left.t \in\right] 0, T[:$

$$
\begin{aligned}
\rho \int_{\Omega} \frac{d \boldsymbol{v}}{d t} \cdot \boldsymbol{\varphi} \mathrm{d} x+\mu \int_{\Omega_{f}} \nabla \boldsymbol{v}: \nabla \boldsymbol{\varphi} \mathrm{d} x+\int_{\Omega_{p}} \widetilde{\mu}^{p} \nabla \boldsymbol{v}: \nabla \boldsymbol{\varphi} \mathrm{d} x+\mu \int_{\Omega_{p}}\left(\boldsymbol{K}^{-1} \boldsymbol{v}\right) \cdot \boldsymbol{\varphi} \mathrm{d} x \\
\quad-\frac{2 \mu}{\sqrt{K}} \sum_{j=1}^{d-1} \int_{\Sigma} \alpha \llbracket \boldsymbol{v} \cdot \boldsymbol{\tau}_{j} \rrbracket_{\Sigma} \boldsymbol{\varphi}_{\mid \Sigma}^{p} \cdot \boldsymbol{\tau}_{j} \mathrm{~d} s=\rho \int_{\Omega} \boldsymbol{f} \cdot \boldsymbol{\varphi} \mathrm{d} x .
\end{aligned}
$$


From the other side, using the set of now recovered equations (18) with the pressure field $q$ to form the weak problem with the same test function $\varphi$ as before, we have using Eq. (20) :

$$
\begin{aligned}
\rho \int_{\Omega} \frac{d \boldsymbol{v}}{d t} \cdot \boldsymbol{\varphi} \mathrm{d} x+\mu \int_{\Omega_{f}} \nabla \boldsymbol{v}: \nabla \boldsymbol{\varphi} \mathrm{d} x+\int_{\Omega_{p}} \widetilde{\mu}^{p} \nabla \boldsymbol{v}: \nabla \boldsymbol{\varphi} \mathrm{d} x+\mu \int_{\Omega_{p}}\left(\boldsymbol{K}^{-1} \boldsymbol{v}\right) \cdot \boldsymbol{\varphi} \mathrm{d} x \\
\quad-2 \sum_{j=1}^{d-1}\left\langle\overline{\left.\boldsymbol{\sigma}(\boldsymbol{v}, q) \cdot \boldsymbol{n}_{\Sigma} \cdot \boldsymbol{\tau}_{j}, \boldsymbol{\varphi}_{\mid \Sigma}^{p} \cdot \boldsymbol{\tau}_{j}\right\rangle_{-1 / 2, \Sigma}=\rho \int_{\Omega} \boldsymbol{f} \cdot \boldsymbol{\varphi} \mathrm{d} x .}\right.
\end{aligned}
$$

Then, by differencing the two previous equations considering the orthogonal components in the tangent plane to $\Sigma$, we get :

$$
\left\langle{\overline{\boldsymbol{\sigma}(\boldsymbol{v}, q) \cdot \boldsymbol{n}_{\Sigma}}}_{\boldsymbol{\tau}} \boldsymbol{\tau}_{j}, \boldsymbol{\varphi}_{\mid \Sigma}^{p} \cdot \boldsymbol{\tau}_{j}\right\rangle_{-1 / 2, \Sigma}=\frac{\mu}{\sqrt{K}} \int_{\Sigma} \alpha \llbracket \boldsymbol{v} \cdot \boldsymbol{\tau}_{j} \rrbracket_{\Sigma} \boldsymbol{\varphi}_{\mid \Sigma}^{p} \cdot \boldsymbol{\tau}_{j} \mathrm{~d} s, \quad \text { for all } 1 \leq j \leq d-1 .
$$

Let us now take any $\boldsymbol{\psi} \in H^{1 / 2}(\Sigma)^{d}$. By applying Lemma 2.4 in the domain $\Omega_{p}$, there exists $\varphi^{p} \in H^{1}\left(\Omega_{p}\right)^{d}$ such that $\nabla \cdot \varphi^{p}=0$ in $\Omega_{p}$ and

$$
\boldsymbol{\psi}=\boldsymbol{\varphi}_{\mid \Sigma}^{p}+\frac{1}{|\Sigma|}\left(\int_{\Sigma} \boldsymbol{\psi} \cdot \boldsymbol{n} \mathrm{d} s\right) \boldsymbol{n}, \quad \text { where }|\Sigma| \text { is the }(d-1) \text { measure of } \Sigma
$$

We have then : $\boldsymbol{\psi} \cdot \boldsymbol{\tau}_{j}=\boldsymbol{\varphi}_{\mid \Sigma}^{p} \cdot \boldsymbol{\tau}_{j}$ for all $1 \leq j \leq d-1$.

Hence, the previous equation also reads :

$$
\left\langle\overline{\boldsymbol{\sigma}(\boldsymbol{v}, q) \cdot \boldsymbol{n}_{\Sigma}} \cdot \boldsymbol{\tau}_{j}, \boldsymbol{\psi} \cdot \boldsymbol{\tau}_{j}\right\rangle_{-1 / 2, \Sigma}=\frac{\mu}{\sqrt{K}} \int_{\Sigma} \alpha \llbracket \boldsymbol{v} \cdot \boldsymbol{\tau}_{j} \rrbracket_{\Sigma} \boldsymbol{\psi} \cdot \boldsymbol{\tau}_{j} \mathrm{~d} s, \quad \text { for all } 1 \leq j \leq d-1 \quad \forall \boldsymbol{\psi} \in H^{1 / 2}(\Sigma)^{d}
$$

This means that

$$
{\overline{\boldsymbol{\sigma}(\boldsymbol{v}, q) \cdot \boldsymbol{n}_{\Sigma}}}_{\boldsymbol{\tau}_{j}}=\frac{\mu}{\sqrt{K}} \alpha \llbracket \boldsymbol{v} \cdot \boldsymbol{\tau}_{j} \rrbracket_{\Sigma}, \quad \text { for all } 1 \leq j \leq d-1
$$

and $(\boldsymbol{v}, q)$ satisfies the velocity jump interface condition in (19).

We proceed similarly to recover the last interface condition of stress jump in (19). We choose any test function $\boldsymbol{\varphi} \in \boldsymbol{W}$ such that $\llbracket \varphi \rrbracket_{\Sigma}=0$, i.e. $\boldsymbol{\varphi}_{\mid \Sigma}^{f}=\boldsymbol{\varphi}_{\mid \Sigma}^{p}=\boldsymbol{\varphi}_{\mid \Sigma}$ and thus $\overline{\boldsymbol{\varphi}}_{\Sigma}=\boldsymbol{\varphi}_{\mid \Sigma}$. It means in fact that $\boldsymbol{\varphi}$ is arbitrary in $\boldsymbol{V}$. From one side, since $\boldsymbol{v}$ verifies the weak form (22), we have for a.e. $t \in] 0, T[$ :

$$
\begin{aligned}
\rho \int_{\Omega} \frac{d \boldsymbol{v}}{d t} \cdot \boldsymbol{\varphi} \mathrm{d} x+\mu \int_{\Omega_{f}} \nabla \boldsymbol{v}: \nabla \boldsymbol{\varphi} \mathrm{d} x+\int_{\Omega_{p}} \widetilde{\mu}^{p} \nabla \boldsymbol{v}: \nabla \boldsymbol{\varphi} \mathrm{d} x & +\mu \int_{\Omega_{p}}\left(\boldsymbol{K}^{-1} \boldsymbol{v}\right) \cdot \boldsymbol{\varphi} \mathrm{d} x \\
& \quad+\frac{\mu}{\sqrt{K}} \int_{\Sigma}\left(\boldsymbol{\beta} \overline{\boldsymbol{v}}_{\Sigma}\right) \cdot \boldsymbol{\varphi} \mid \Sigma \mathrm{d} s=\rho \int_{\Omega} \boldsymbol{f} \cdot \boldsymbol{\varphi} \mathrm{d} x
\end{aligned}
$$

From the other side, using the set of now recovered equations (18) with the pressure field $q$ to form the weak problem with the same test function $\boldsymbol{\varphi} \in \boldsymbol{V}$, we have using Eq. $(20), \llbracket \varphi \rrbracket_{\Sigma}=0$ and $\overline{\boldsymbol{\varphi}}_{\Sigma}=\boldsymbol{\varphi}_{\mid \Sigma}:$

$$
\begin{aligned}
\rho \int_{\Omega} \frac{d \boldsymbol{v}}{d t} \cdot \boldsymbol{\varphi} \mathrm{d} x+\mu \int_{\Omega_{f}} \nabla \boldsymbol{v}: \nabla \boldsymbol{\varphi} \mathrm{d} x+\int_{\Omega_{p}} \widetilde{\mu}^{p} \nabla \boldsymbol{v}: \nabla \boldsymbol{\varphi} \mathrm{d} x & +\mu \int_{\Omega_{p}}\left(\boldsymbol{K}^{-1} \boldsymbol{v}\right) \cdot \boldsymbol{\varphi} \mathrm{d} x \\
& +\left\langle\llbracket \boldsymbol{\sigma}(\boldsymbol{v}, q) \cdot \boldsymbol{n} \rrbracket_{\Sigma}, \boldsymbol{\varphi} \mid \Sigma\right\rangle_{-1 / 2, \Sigma}=\rho \int_{\Omega} \boldsymbol{f} \cdot \boldsymbol{\varphi} \mathrm{d} x
\end{aligned}
$$


Then, by differencing the two previous equations, we get :

$$
\left\langle\llbracket \boldsymbol{\sigma}(\boldsymbol{v}, q) \cdot \boldsymbol{n} \rrbracket_{\Sigma}, \boldsymbol{\varphi}_{\mid \Sigma}\right\rangle_{-1 / 2, \Sigma}=\frac{\mu}{\sqrt{K}} \int_{\Sigma}\left(\boldsymbol{\beta} \overline{\boldsymbol{v}}_{\Sigma}\right) \cdot \boldsymbol{\varphi}_{\mid \Sigma} \mathrm{d} s, \quad \text { for all } \boldsymbol{\varphi} \in \boldsymbol{V} .
$$

Since $\Sigma$ is a closed surface, this is also equivalent to :

$$
\left\langle\llbracket \boldsymbol{\sigma}(\boldsymbol{v}, q) \cdot \boldsymbol{n} \rrbracket_{\Sigma}-\frac{\mu}{\sqrt{K}} \boldsymbol{\beta} \overline{\boldsymbol{v}}_{\Sigma}, \boldsymbol{\varphi}_{\mid \Sigma}\right\rangle_{-1 / 2, \Sigma}=0, \quad \text { for all } \boldsymbol{\varphi} \in H^{1}\left(\Omega_{p}\right)^{d} ; \quad \boldsymbol{\nabla} \cdot \boldsymbol{\varphi}=0 \text { in } \Omega_{p} .
$$

Let us now take any $\boldsymbol{\psi} \in H^{1 / 2}(\Sigma)^{d}$. By applying Lemma 2.4 in the domain $\Omega_{p}$, there exists $\varphi \in H^{1}\left(\Omega_{p}\right)^{d}$ with $\boldsymbol{\nabla} \cdot \boldsymbol{\varphi}=0$ in $\Omega_{p}$ such that

$$
\boldsymbol{\psi}=\boldsymbol{\varphi}_{\mid \Sigma}+\frac{1}{|\Sigma|}\left(\int_{\Sigma} \boldsymbol{\psi} \cdot \boldsymbol{n} \mathrm{d} s\right) \boldsymbol{n}, \quad \text { where }|\Sigma| \text { is the }(d-1) \text { measure of } \Sigma .
$$

Then it yields, the first term in the r.h.s. vanishing with the previous equation :

$$
\begin{aligned}
\left\langle\llbracket \boldsymbol{\sigma}(\boldsymbol{v}, q) \cdot \boldsymbol{n} \rrbracket_{\Sigma}-\frac{\mu}{\sqrt{K}} \boldsymbol{\beta} \overline{\boldsymbol{v}}_{\Sigma}, \boldsymbol{\psi}\right\rangle_{-1 / 2, \Sigma} & =\left\langle\llbracket \boldsymbol{\sigma}(\boldsymbol{v}, q) \cdot \boldsymbol{n} \rrbracket_{\Sigma}-\frac{\mu}{\sqrt{K}} \boldsymbol{\beta} \overline{\boldsymbol{v}}_{\Sigma}, \boldsymbol{\varphi}_{\mid \Sigma}\right\rangle_{-1 / 2, \Sigma} \\
& +\frac{1}{|\Sigma|}\left\langle\llbracket \boldsymbol{\sigma}(\boldsymbol{v}, q) \cdot \boldsymbol{n} \rrbracket_{\Sigma}-\frac{\mu}{\sqrt{K}} \boldsymbol{\beta} \overline{\boldsymbol{v}}_{\Sigma}, \boldsymbol{n}\right\rangle_{-1 / 2, \Sigma} \int_{\Sigma} \boldsymbol{\psi} \cdot \boldsymbol{n} \mathrm{d} s \\
& =\frac{1}{|\Sigma|}\left\langle\llbracket \boldsymbol{\sigma}(\boldsymbol{v}, q) \cdot \boldsymbol{n} \rrbracket_{\Sigma}-\frac{\mu}{\sqrt{K}} \boldsymbol{\beta} \overline{\boldsymbol{v}}_{\Sigma}, \boldsymbol{n}\right\rangle_{-1 / 2, \Sigma} \int_{\Sigma} \boldsymbol{\psi} \cdot \boldsymbol{n} \mathrm{d} s, \quad \forall \boldsymbol{\psi} \in H^{1 / 2}(\Sigma)^{d} .
\end{aligned}
$$

Defining the constant quantity $\Pi_{0}$ by

$$
\Pi_{0}:=\frac{1}{|\Sigma|}\left\langle\llbracket \boldsymbol{\sigma}(\boldsymbol{v}, q) \cdot \boldsymbol{n} \rrbracket_{\Sigma}-\frac{\mu}{\sqrt{K}} \boldsymbol{\beta} \overline{\boldsymbol{v}}_{\Sigma}, \boldsymbol{n}\right\rangle_{-1 / 2, \Sigma}
$$

we get :

$$
\begin{aligned}
\left\langle\llbracket \boldsymbol{\sigma}(\boldsymbol{v}, q) \cdot \boldsymbol{n} \rrbracket_{\Sigma}-\frac{\mu}{\sqrt{K}} \boldsymbol{\beta} \overline{\boldsymbol{v}}_{\Sigma}, \boldsymbol{\psi}\right\rangle_{-1 / 2, \Sigma} & =\Pi_{0} \int_{\Sigma} \boldsymbol{\psi} \cdot \boldsymbol{n} \mathrm{d} s=\int_{\Sigma} \Pi_{0} \boldsymbol{n} \cdot \boldsymbol{\psi} \mathrm{d} s \\
& =\left\langle\Pi_{0} \boldsymbol{n}, \boldsymbol{\psi}\right\rangle_{-1 / 2, \Sigma}, \quad \forall \boldsymbol{\psi} \in H^{1 / 2}(\Sigma)^{d} .
\end{aligned}
$$

Now, the pressure field $p$ in $\Omega$ can be defined as

$$
p_{\mid \Omega_{f}}:=q^{f}+\frac{\Pi_{0}}{2}, \quad p_{\mid \Omega_{p}}:=q^{p}-\frac{\Pi_{0}}{2}, \quad \text { such that } \llbracket p-q \rrbracket_{\Sigma}=\Pi_{0} .
$$

This is allowed because both $q^{f}$ and $q^{p}$ are defined up to an additive constant. By this way, we finally get :

$$
\begin{aligned}
\left\langle\llbracket \boldsymbol{\sigma}(\boldsymbol{v}, p) \cdot \boldsymbol{n} \rrbracket_{\Sigma}-\frac{\mu}{\sqrt{K}} \boldsymbol{\beta} \overline{\boldsymbol{v}}_{\Sigma}, \boldsymbol{\psi}\right\rangle_{-1 / 2, \Sigma} & =\left\langle\llbracket \boldsymbol{\sigma}(\boldsymbol{v}, q) \cdot \boldsymbol{n} \rrbracket_{\Sigma}-\Pi_{0} \boldsymbol{n}-\frac{\mu}{\sqrt{K}} \boldsymbol{\beta} \overline{\boldsymbol{v}}_{\Sigma}, \boldsymbol{\psi}\right\rangle_{-1 / 2, \Sigma} \\
& =0, \quad \forall \boldsymbol{\psi} \in H^{1 / 2}(\Sigma)^{d} .
\end{aligned}
$$

This means that

$$
\llbracket \boldsymbol{\sigma}(\boldsymbol{v}, p) \cdot \boldsymbol{n} \rrbracket_{\Sigma}=\frac{\mu}{\sqrt{K}} \boldsymbol{\beta} \overline{\boldsymbol{v}}_{\Sigma}
$$

and $(\boldsymbol{v}, p)$ satisfies the stress jump interface condition in (19). Finally, since we have: $\overline{(p-q)}_{\Sigma}=0$, it is clear 
that $(\boldsymbol{v}, p)$ also verifies the velocity jump interface condition in (19) as well as the Stokes/Brinkman equations (18), which concludes the proof.

\section{Stokes/Darcy problem With Jump interface CONDitions}

In this part, the Stokes/Darcy coupled flow problem with the jump interface conditions (14) is proved to be globally well-posed in time following the idea in [8] by using the vanishing viscosity method and passing to the limit when $\widetilde{\mu}^{p} \rightarrow 0$ inside $\Omega_{p}$ in the Stokes/Brinkman problem (18-19). In [8], the analysis was briefly carried out for the steady flow with a similar set of jump embedded boundary conditions.

Hence, the problem reads as below in the time-space domain $(0, T) \times \Omega$ for any $T>0$ and all $\varepsilon>0$ :

$$
\left\{\begin{aligned}
\boldsymbol{\nabla} \cdot \boldsymbol{v}_{\varepsilon} & =0 & & \text { in }(0, T) \times \Omega, \\
\rho \partial_{t} \boldsymbol{v}_{\varepsilon}-\boldsymbol{\nabla} \cdot \boldsymbol{\sigma}\left(\boldsymbol{v}_{\varepsilon}, p_{\varepsilon}\right)^{f} & =\rho \boldsymbol{f} & & \text { in }(0, T) \times \Omega_{f}, \\
\rho \partial_{t} \boldsymbol{v}_{\varepsilon}-\boldsymbol{\nabla} \cdot \boldsymbol{\sigma}\left(\boldsymbol{v}_{\varepsilon}, p_{\varepsilon}\right)^{p}+\mu \boldsymbol{K}^{-1} \boldsymbol{v}_{\varepsilon} & =\rho \boldsymbol{f} & & \text { in }(0, T) \times \Omega_{p}, \\
\boldsymbol{v}_{\varepsilon} & =0 & & \text { on }(0, T) \times \Gamma, \\
\boldsymbol{v}_{\varepsilon}(t=0) & =\boldsymbol{v}_{0} \in \boldsymbol{W} & & \text { in } \Omega,
\end{aligned}\right.
$$

where :

$$
\begin{gathered}
\boldsymbol{\sigma}\left(\boldsymbol{v}_{\varepsilon}, p_{\varepsilon}\right):=\widetilde{\mu} \nabla \boldsymbol{v}_{\varepsilon}-p_{\varepsilon} \boldsymbol{I}, \quad \text { with } \quad \phi^{f}:=1, \quad \widetilde{\mu}^{f}:=\mu, \quad \widetilde{\mu}^{p}:=\varepsilon, \\
\left\{\begin{array}{c}
\llbracket \boldsymbol{v}_{\varepsilon} \cdot \boldsymbol{n} \rrbracket_{\Sigma}=0, \\
\frac{\boldsymbol{\sigma}\left(\boldsymbol{v}_{\varepsilon}, p_{\varepsilon}\right) \cdot \boldsymbol{n}_{\Sigma} \cdot \boldsymbol{\tau}_{j}=}{\widetilde{\mu} \nabla \boldsymbol{v}_{\varepsilon} \cdot \boldsymbol{n}_{\Sigma} \cdot \boldsymbol{\tau}_{j}=\mu \frac{\alpha}{\sqrt{K}} \llbracket \boldsymbol{v}_{\varepsilon} \cdot \boldsymbol{\tau}_{j} \rrbracket_{\Sigma}, \quad 1 \leq j \leq d-1 \quad \text { on }(0, T) \times \Sigma .} \\
\llbracket \boldsymbol{\sigma}\left(\boldsymbol{v}_{\varepsilon}, p_{\varepsilon}\right) \cdot \boldsymbol{n} \rrbracket_{\Sigma}=\frac{\mu}{\sqrt{K}} \boldsymbol{\beta} \overline{\boldsymbol{v}}_{\varepsilon},
\end{array}\right.
\end{gathered}
$$

Using Eq. (22), since $\boldsymbol{v}_{\varepsilon}^{\prime} \in L^{2}(0, T ; \boldsymbol{H})$ as soon as $\boldsymbol{v}_{0} \in \boldsymbol{W}$ from Section 2, the problem (27-28) admits the weak form below :

$$
\begin{aligned}
\rho \int_{\Omega} \frac{d \boldsymbol{v}_{\varepsilon}}{d t} \cdot \boldsymbol{\varphi} \mathrm{d} x+\mu \int_{\Omega_{f}} \nabla \boldsymbol{v}_{\varepsilon}: \nabla \boldsymbol{\varphi} \mathrm{d} x & +\varepsilon \int_{\Omega_{p}} \nabla \boldsymbol{v}_{\varepsilon}: \nabla \boldsymbol{\varphi} \mathrm{d} x+\mu \int_{\Omega_{p}}\left(\boldsymbol{K}^{-1} \boldsymbol{v}_{\varepsilon}\right) \cdot \boldsymbol{\varphi} \mathrm{d} x \\
& +\frac{\mu}{\sqrt{K}} \int_{\Sigma} \alpha \llbracket \boldsymbol{v}_{\varepsilon} \rrbracket_{\Sigma} \cdot \llbracket \boldsymbol{\varphi} \rrbracket_{\Sigma} \mathrm{d} s+\frac{\mu}{\sqrt{K}} \int_{\Sigma}\left(\boldsymbol{\beta} \overline{\boldsymbol{v}_{\varepsilon}}\right) \cdot \overline{\boldsymbol{\varphi}}_{\Sigma} \mathrm{d} s=\rho \int_{\Omega} \boldsymbol{f} \cdot \boldsymbol{\varphi} \mathrm{d} x \\
& \text { for a.e. } t \in] 0, T[\quad \forall \boldsymbol{\varphi} \in \boldsymbol{W} .
\end{aligned}
$$

\subsection{Uniform energy bounds and weak limits}

By Theorem 2.5 with $\boldsymbol{v}_{0} \in \boldsymbol{W}$, the solution to (27-28) satisfies for all $\varepsilon>0: \boldsymbol{v}_{\varepsilon} \in \mathcal{C}([0, T] ; \boldsymbol{H}) \cap L^{2}(0, T ; \boldsymbol{W})$ with $\boldsymbol{v}_{\varepsilon}^{\prime} \in L^{2}(0, T ; \boldsymbol{H})$. From the energy equality (23), we have for all $t \in[0, T]$ using the basic assumptions on 
the data and the Cauchy-Schwarz inequality in the right-hand side :

$$
\begin{aligned}
\frac{\rho}{2}\left\|\boldsymbol{v}_{\varepsilon}(t)\right\|_{0, \Omega}^{2}+\mu \int_{0}^{t}\left\|\nabla \boldsymbol{v}_{\varepsilon}\right\|_{0, \Omega_{f}}^{2} \mathrm{~d} \tau & +\varepsilon \int_{0}^{t}\left\|\nabla \boldsymbol{v}_{\varepsilon}\right\|_{0, \Omega_{p}}^{2} \mathrm{~d} \tau+\mu k_{m} \int_{0}^{t}\left\|\boldsymbol{v}_{\varepsilon}\right\|_{0, \Omega_{p}}^{2} \mathrm{~d} \tau \\
& +\frac{\mu \alpha_{m}}{\sqrt{K}} \int_{0}^{t}\left\|\llbracket \boldsymbol{v}_{\varepsilon} \rrbracket_{\Sigma}\right\|_{0, \Sigma}^{2} \mathrm{~d} \tau+\frac{\mu \beta_{m}}{\sqrt{K}} \int_{0}^{t}\left\|\overline{\boldsymbol{v}}_{\varepsilon \Sigma}\right\|_{0, \Sigma}^{2} \mathrm{~d} \tau \\
& \leq \frac{\rho}{2}\left\|\boldsymbol{v}_{0}\right\|_{0, \Omega}^{2}+\frac{\rho}{2} \int_{0}^{t}\|\boldsymbol{f}\|_{0, \Omega}^{2} \mathrm{~d} \tau+\frac{\rho}{2} \int_{0}^{t}\left\|\boldsymbol{v}_{\varepsilon}(\tau)\right\|_{0, \Omega}^{2} \mathrm{~d} \tau
\end{aligned}
$$

Then applying the classical Gronwall lemma to deal with the last term in the right-hand side, we get the uniform energy estimate below :

$$
\begin{aligned}
& \frac{\rho}{2}\left\|\boldsymbol{v}_{\varepsilon}(t)\right\|_{0, \Omega}^{2}+\mu \int_{0}^{t}\left\|\nabla \boldsymbol{v}_{\varepsilon}\right\|_{0, \Omega_{f}}^{2} \mathrm{~d} \tau+\varepsilon \int_{0}^{t}\left\|\nabla \boldsymbol{v}_{\varepsilon}\right\|_{0, \Omega_{p}}^{2} \mathrm{~d} \tau+\mu k_{m} \int_{0}^{t}\left\|\boldsymbol{v}_{\varepsilon}\right\|_{0, \Omega_{p}}^{2} \mathrm{~d} \tau \\
&+\frac{\mu \alpha_{m}}{\sqrt{K}} \int_{0}^{t}\left\|\llbracket \boldsymbol{v}_{\varepsilon} \rrbracket_{\Sigma}\right\|_{0, \Sigma}^{2} \mathrm{~d} \tau+\frac{\mu \beta_{m}}{\sqrt{K}} \int_{0}^{t}\left\|\overline{\boldsymbol{v}_{\varepsilon} \Sigma}\right\|_{0, \Sigma}^{2} \mathrm{~d} \tau \\
& \leq C_{0}\left(\Omega, T, \rho, \boldsymbol{v}_{0}, \boldsymbol{f}\right) .
\end{aligned}
$$

Using (26) with $\varepsilon \leq 1$, we also get the uniform bound below for the time derivative for a.e. $t \in[0, T]$ :

$$
\begin{aligned}
\rho \int_{0}^{t}\left\|\frac{d \boldsymbol{v}_{\varepsilon}}{d t}(\tau)\right\|_{0, \Omega}^{2} \mathrm{~d} \tau+\mu\left\|\nabla \boldsymbol{v}_{\varepsilon}(t)\right\|_{0, \Omega_{f}}^{2} & +\varepsilon\left\|\nabla \boldsymbol{v}_{\varepsilon}(t)\right\|_{0, \Omega_{p}}^{2}+\mu k_{m}\left\|\boldsymbol{v}_{\varepsilon}(t)\right\|_{0, \Omega_{p}}^{2} \\
& +\frac{\mu \alpha_{m}}{\sqrt{K}}\left\|\llbracket \boldsymbol{v}_{\varepsilon}(t) \rrbracket_{\Sigma}\right\|_{0, \Sigma}^{2}+\frac{\mu \beta_{m}}{\sqrt{K}} \| \overline{\boldsymbol{v}_{\varepsilon}(t)} \\
& \|_{0, \Sigma}^{2} \\
\leq & \mu\left\|\nabla \boldsymbol{v}_{0}\right\|_{0, \Omega_{f}}^{2}+\left\|\nabla \boldsymbol{v}_{0}\right\|_{0, \Omega_{p}}^{2}+\mu k_{M}\left\|\boldsymbol{v}_{0}\right\|_{0, \Omega_{p}}^{2} \\
& +\frac{\mu \alpha_{M}}{\sqrt{K}}\left\|\llbracket \boldsymbol{v}_{0} \rrbracket_{\Sigma}\right\|_{0, \Sigma}^{2}+\frac{\mu \beta_{M}}{\sqrt{K}}\left\|\overline{\boldsymbol{v}_{0 \Sigma}}\right\|_{0, \Sigma}^{2}+\rho \int_{0}^{t}\|\boldsymbol{f}(\tau)\|_{0, \Omega}^{2} \mathrm{~d} \tau, \\
& \leq C_{1}\left(\Omega, \Omega_{f}, \Omega_{p}, T, \rho, \mu, \boldsymbol{K}, \alpha, \boldsymbol{\beta}, \boldsymbol{v}_{0}, \boldsymbol{f}\right) .
\end{aligned}
$$

Hence, applying Banach compactness theorem for the weak topologies, see e.g. [20, Chap. III], with the uniform bounds given in (30-31), there exists $\boldsymbol{v} \in L^{\infty}(0, T ; \boldsymbol{H})$ with $\boldsymbol{v}^{f} \in L^{2}\left(0, T ; H_{0 \Gamma}^{1}\left(\Omega_{f}\right)^{d}\right)$ verifying $\boldsymbol{v}_{\mid \Gamma}^{f}=0$ a.e. in $(0, T)$ and $\frac{d \boldsymbol{v}}{d t} \in L^{2}(0, T ; \boldsymbol{H})$, and there exists $\widetilde{\boldsymbol{v}} \in L^{\infty}\left(0, T ; L^{2}(\Omega)^{d}\right)$ with $\boldsymbol{h}:=\nabla \widetilde{\boldsymbol{v}}^{p} \in L^{2}\left(0, T ; L^{2}\left(\Omega_{p}\right)^{d \times d}\right)$ such that, up to some subsequences, we have the weak limits below when $\varepsilon \rightarrow 0$ :

$a) \bullet \quad \boldsymbol{v}_{\varepsilon} \rightarrow \boldsymbol{v}$ in $L^{\infty}(0, T ; \boldsymbol{H})$ weak $\star$, and in $L^{2}(0, T ; \boldsymbol{H})$ weak,

$b) \bullet \nabla \boldsymbol{v}_{\varepsilon}^{f} \rightarrow \nabla \boldsymbol{v}^{f}$ in $L^{2}\left(0, T ; L^{2}\left(\Omega_{f}\right)^{d \times d}\right)$ weak,

c) $\sqrt{\varepsilon} \nabla \boldsymbol{v}_{\varepsilon}^{p} \rightarrow \boldsymbol{h}:=\nabla \widetilde{\boldsymbol{v}}^{p}$ in $L^{2}\left(0, T ; L^{2}\left(\Omega_{p}\right)^{d \times d}\right)$ weak,

$d) \bullet \quad \frac{d \boldsymbol{v}_{\varepsilon}}{d t} \rightarrow \frac{d \boldsymbol{v}}{d t}$ in $L^{2}(0, T ; \boldsymbol{H})$ weak.

In the two first points $a$ ) and $b$ ) above, the same limit $\boldsymbol{v}$ is recovered since the weak convergence implies the convergence of $\boldsymbol{v}_{\varepsilon}$ in the distribution sense in $\mathcal{D}(] 0, T[\times \Omega)^{\prime}$ and thus, the limit being unique, the same limit is reached. For the fourth point $d$ ), it is also true because the time derivative operator is continuous in the distribution sense. 
Besides, using Aubin-Lions compactness theorem [49, Chap. 1] since the continuous injection $H^{1}\left(\Omega_{f}\right) \hookrightarrow L^{2}\left(\Omega_{f}\right)$ is compact by Rellich's theorem, the sequence $\left(\boldsymbol{v}_{\varepsilon}^{f}\right)_{\varepsilon>0}$ converges strongly to $\boldsymbol{v}^{f}$ in $L^{2}\left(0, T ; L^{2}\left(\Omega_{f}\right)^{d}\right)$.

In particular, $\boldsymbol{v}_{0}=\boldsymbol{v}_{\varepsilon}(0) \rightarrow \boldsymbol{v}(0)$ in $\boldsymbol{W}^{\prime}$ strongly when $\varepsilon \rightarrow 0$. But the sequence $\boldsymbol{v}_{\varepsilon}(0)$ also converges weakly in $\boldsymbol{H}$ and thus in $\boldsymbol{W}^{\prime}$ towards a limit which is $\boldsymbol{v}_{0}$ with the initial condition in (27). By uniqueness of the limit in $\boldsymbol{W}^{\prime}$, we get that: $\boldsymbol{v}(0)=\boldsymbol{v}_{0}$, i.e. the limit solution satisfies the desired initial condition.

Now concerning the velocity traces on $\Sigma$, since $\boldsymbol{v}_{\varepsilon}^{f}$ converges weakly towards $\boldsymbol{v}^{f}$ in $L^{2}\left(0, T ; H^{1}\left(\Omega_{f}\right)^{d}\right)$, we have from the trace continuity on $\Sigma$ :

$e) \bullet \quad \boldsymbol{v}_{\varepsilon \mid \Sigma}^{f} \rightarrow \boldsymbol{v}_{\mid \Sigma}^{f}$ in $L^{2}\left(0, T ; H^{1 / 2}(\Sigma)^{d}\right)$ weak, and thus also in $L^{2}\left(0, T ; L^{2}(\Sigma)^{d}\right)$ weak.

Then, from the uniform bounds in (30), both $\llbracket \boldsymbol{v}_{\varepsilon} \rrbracket_{\Sigma}$ and $\overline{\boldsymbol{v}}_{\varepsilon \Sigma}$ admit a weak limit in $L^{2}\left(0, T ; L^{2}(\Sigma)^{d}\right)$. Hence, there exists $\boldsymbol{v}_{\Sigma}^{\star} \in L^{2}\left(0, T ; L^{2}(\Sigma)^{d}\right)$ such that :

$f) \bullet \quad \llbracket \boldsymbol{v}_{\varepsilon} \rrbracket_{\Sigma} \rightarrow \llbracket \boldsymbol{v} \rrbracket_{\Sigma}^{\star}:=\left(\boldsymbol{v}_{\mid \Sigma}^{f}-\boldsymbol{v}_{\Sigma}^{\star}\right)$ in $L^{2}\left(0, T ; L^{2}(\Sigma)^{d}\right)$ weak,

$g) \bullet \quad \overline{\boldsymbol{v}}_{\varepsilon \Sigma} \rightarrow \overline{\boldsymbol{v}}_{\Sigma}^{\star}:=\left(\boldsymbol{v}_{\mid \Sigma}^{f}+\boldsymbol{v}_{\Sigma}^{\star}\right) / 2$ in $L^{2}\left(0, T ; L^{2}(\Sigma)^{d}\right)$ weak,

$h) \bullet \quad 0=\llbracket \boldsymbol{v}_{\varepsilon} \cdot \boldsymbol{n} \rrbracket_{\Sigma} \rightarrow 0=\llbracket \boldsymbol{v} \cdot \boldsymbol{n} \rrbracket_{\Sigma}:=\left(\boldsymbol{v}^{f} \cdot \boldsymbol{n}_{\mid \Sigma}-\boldsymbol{v}^{p} \cdot \boldsymbol{n}_{\mid \Sigma}\right)$ weak, $\boldsymbol{v}_{\varepsilon}^{f} \cdot \boldsymbol{n}_{\mid \Sigma}=\overline{\boldsymbol{v}}_{\varepsilon} \cdot \boldsymbol{n}_{\Sigma} \rightarrow \overline{\boldsymbol{v} \cdot \boldsymbol{n}_{\Sigma}}=\boldsymbol{v}^{f} \cdot \boldsymbol{n}_{\mid \Sigma}$ in $L^{2}\left(0, T ; L^{2}(\Sigma)\right)$ weak

since $\boldsymbol{v}^{p}(t) \in \boldsymbol{H}_{\text {div }}\left(\Omega_{p}\right)$ and then admits a normal trace in a weak sense $\boldsymbol{v}^{p} \cdot \boldsymbol{n}_{\mid \Sigma} \in L^{2}\left(0, T ; H^{-1 / 2}(\Sigma)\right)$. Thus we have in fact: $\boldsymbol{v}_{\Sigma}^{\star} \cdot \boldsymbol{n}=\boldsymbol{v}^{p} \cdot \boldsymbol{n}_{\mid \Sigma}=\boldsymbol{v}^{f} \cdot \boldsymbol{n}_{\mid \Sigma}$ and belongs to $L^{2}\left(0, T ; H^{1 / 2}(\Sigma)\right)$.

\subsection{Limit problem and interpretation}

Since the problem is linear and the coefficients of the data being bounded functions, it is classical that the previous weak limits are sufficient to take the limit of (29) when $\varepsilon \rightarrow 0$. Therefore, we get that $\boldsymbol{v} \in L^{\infty}(0, T ; \boldsymbol{H})$

with $\boldsymbol{v}^{f} \in L^{2}\left(0, T ; H_{0 \Gamma}^{1}\left(\Omega_{f}\right)^{d}\right)$ and $\frac{d \boldsymbol{v}}{d t} \in L^{2}(0, T ; \boldsymbol{H})$ and such that $\boldsymbol{v}(0)=\boldsymbol{v}_{0}$ in $\boldsymbol{H}$ satisfies the following limit weak problem :

$$
\begin{aligned}
\rho \int_{\Omega} \frac{d \boldsymbol{v}}{d t} \cdot \boldsymbol{\varphi} \mathrm{d} x+\mu \int_{\Omega_{f}} \nabla \boldsymbol{v}: \nabla \boldsymbol{\varphi} \mathrm{d} x & +\mu \int_{\Omega_{p}}\left(\boldsymbol{K}^{-1} \boldsymbol{v}\right) \cdot \boldsymbol{\varphi} \mathrm{d} x \\
& +\frac{\mu}{\sqrt{K}} \int_{\Sigma} \alpha \llbracket \boldsymbol{v} \rrbracket_{\Sigma}^{\star} \cdot \llbracket \boldsymbol{\varphi} \rrbracket_{\Sigma} \mathrm{d} s+\frac{\mu}{\sqrt{K}} \int_{\Sigma}\left(\boldsymbol{\beta} \overline{\boldsymbol{v}}_{\Sigma}^{\star}\right) \cdot \overline{\boldsymbol{\varphi}}_{\Sigma} \mathrm{d} s=\rho \int_{\Omega} \boldsymbol{f} \cdot \boldsymbol{\varphi} \mathrm{d} x,
\end{aligned}
$$

for a.e. $t \in] 0, T[; \quad \forall \boldsymbol{\varphi} \in \boldsymbol{W}$.

Let us now interpret the limit weak problem (32). Proceeding similarly as in the proof of Theorem 2.5 by using De Rham's theorem in $\Omega_{f}$ and in $\Omega_{p}$, there exists a function $q \in L^{2}\left(0, T ; L_{0}^{2}(\Omega)\right)$ with each restriction $q^{f} \in L^{2}\left(0, T ; L_{0}^{2}\left(\Omega_{f}\right)\right)$ and $q^{p} \in L^{2}\left(0, T ; L_{0}^{2}\left(\Omega_{p}\right)\right)$ both defined uniquely up to an additive constant, such that $\boldsymbol{v}$ satisfies the Stokes or Darcy equations in $\Omega_{f}$ or $\Omega_{p}$, respectively :

$$
\begin{aligned}
\boldsymbol{\nabla} \cdot \boldsymbol{v} & =0, & & \text { in }(0, T) \times \Omega, \\
\rho \frac{\partial \boldsymbol{v}}{\partial t}-\mu \Delta \boldsymbol{v}+\nabla q^{f} & =\rho \boldsymbol{f}, & & \text { in }(0, T) \times \Omega_{f}, \\
\rho \frac{\partial \boldsymbol{v}}{\partial t}+\mu \boldsymbol{K}^{-1} \boldsymbol{v}+\nabla q^{p} & =\rho \boldsymbol{f}, & & \text { in }(0, T) \times \Omega_{p} .
\end{aligned}
$$

Moreover, from the regularity of the terms in the last Darcy equation, it is clear that $\nabla q^{p} \in L^{2}\left(0, T ; L^{2}\left(\Omega_{p}\right)^{d}\right)$ and thus $q^{p} \in L^{2}\left(0, T ; L_{0}^{2}\left(\Omega_{p}\right) \cap H^{1}\left(\Omega_{p}\right)\right)$. 
Let us look for the jump interface conditions on $\Sigma$ since we have seen that $\llbracket \boldsymbol{v} \cdot \boldsymbol{n} \rrbracket_{\Sigma}=0$. For that, it is interesting to observe that the above Stokes/Darcy equations still admit a general weak form (21) with test functions in $\boldsymbol{W}$ by replacing the former Brinkman's stress vector by Darcy's one which reads :

$$
\boldsymbol{\sigma}(\boldsymbol{v}, q)^{p} \cdot \boldsymbol{n}_{\mid \Sigma}:=-q_{\mid \Sigma}^{p} \boldsymbol{n}
$$

which makes sense since $q^{p}$ has a trace on $\Sigma$ in $L^{2}\left(0, T ; H^{1 / 2}(\Sigma)\right)$. This amounts to formally take $\widetilde{\mu}^{p}=0$ in the previous study of the Stokes/Brinkman problem in Section 2.

Therefore, still following the proof of Theorem 2.5, if we compare the limit weak problem (32) to the general weak form (21) now for the Stokes/Darcy equations, both the velocity jump and stress jump interface conditions are recovered by using Lemma 2.4. More precisely, we get :

$$
\begin{aligned}
{\overline{\boldsymbol{\sigma}}(\boldsymbol{v}, p) \cdot \boldsymbol{n}_{\Sigma}}_{\boldsymbol{\tau}} & =\frac{\mu}{\sqrt{K}} \alpha \llbracket \boldsymbol{v} \cdot \boldsymbol{\tau}_{j} \rrbracket_{\Sigma}^{\star}, \quad \text { for all } 1 \leq j \leq d-1, \\
\llbracket \boldsymbol{\sigma}(\boldsymbol{v}, p) \cdot \boldsymbol{n} \rrbracket_{\Sigma} & =\frac{\mu}{\sqrt{K}} \boldsymbol{\beta} \overline{\boldsymbol{v}}_{\Sigma}^{\star},
\end{aligned}
$$

where the pressure field $p \in L^{2}\left(0, T ; L^{2}(\Omega)\right)$ such that $p^{p} \in L^{2}\left(0, T ; H^{1}\left(\Omega_{p}\right)\right)$ is defined from $(\boldsymbol{v}, q)$ by :

$$
\begin{aligned}
p_{\mid \Omega_{f}}:= & q^{f}+\frac{\Pi_{0}}{2}, \quad p_{\mid \Omega_{p}}:=q^{p}-\frac{\Pi_{0}}{2}, \quad \text { such that } \llbracket p-q \rrbracket_{\Sigma}=\Pi_{0}, \\
& \text { with the constant: } \quad \Pi_{0}:=\frac{1}{|\Sigma|}\left\langle\llbracket \boldsymbol{\sigma}(\boldsymbol{v}, q) \cdot \boldsymbol{n} \rrbracket_{\Sigma}-\frac{\mu}{\sqrt{K}} \boldsymbol{\beta} \overline{\boldsymbol{v}}_{\Sigma}^{\star}, \boldsymbol{n}\right\rangle_{-1 / 2, \Sigma} .
\end{aligned}
$$

Considering the regularity of the different terms in these interface conditions, it is clear that the Stokes stress vector gains an extra regularity and we have here: $\boldsymbol{\sigma}(\boldsymbol{v}, p)^{f} \cdot \boldsymbol{n}_{\mid \Sigma}:=\left(\mu \nabla \boldsymbol{v}^{f} \cdot \boldsymbol{n}-p^{f} \boldsymbol{n}\right)_{\mid \Sigma} \in L^{2}\left(0, T ; L^{2}(\Sigma)^{d}\right)$, since $\boldsymbol{\sigma}(\boldsymbol{v}, p)^{p} \cdot \boldsymbol{n}_{\mid \Sigma}:=-p_{\mid \Sigma}^{p} \boldsymbol{n} \in L^{2}\left(0, T ; L^{2}(\Sigma)^{d}\right)$ and the same for the other term in $\boldsymbol{\beta} \overline{\boldsymbol{v}}_{\Sigma}^{\star}$.

\subsection{Main result of solvability for the Stokes/Darcy problem}

By gathering the results that are shown above in Sections 3.1 and 3.2, let us consider the Stokes/Darcy coupled problem below in the time-space domain $(0, T) \times \Omega$ :

$$
\begin{aligned}
& \left\{\begin{aligned}
\boldsymbol{\nabla} \cdot \boldsymbol{v} & =0 & & \text { in }(0, T) \times \Omega, \\
\rho \partial_{t} \boldsymbol{v}-\mu \Delta \boldsymbol{v}+\nabla p & =\rho \boldsymbol{f} & & \text { in }(0, T) \times \Omega_{f} \\
\rho \partial_{t} \boldsymbol{v}+\mu \boldsymbol{K}^{-1} \boldsymbol{v}+\nabla p & =\rho \boldsymbol{f} & & \text { in }(0, T) \times \Omega_{p} \\
\boldsymbol{v} & =0 & & \text { on }(0, T) \times \Gamma, \\
\boldsymbol{v}(t=0) & =\boldsymbol{v}_{0} & & \text { in } \Omega,
\end{aligned}\right. \\
& \left\{\begin{aligned}
& \llbracket \boldsymbol{v} \cdot \boldsymbol{n} \rrbracket_{\Sigma}=0, \\
& \frac{\boldsymbol{\sigma}(\boldsymbol{v}, p) \cdot \boldsymbol{n}_{\Sigma} \cdot \boldsymbol{\tau}_{j}:=\frac{1}{2} \mu \partial_{n}\left(\boldsymbol{v}^{f} \cdot \boldsymbol{\tau}_{j}\right)_{\mid \Sigma}}{}=\mu \frac{\alpha}{\sqrt{K}} \llbracket \boldsymbol{v} \cdot \boldsymbol{\tau}_{j} \rrbracket_{\Sigma}^{\star}, \quad 1 \leq j \leq d-1 \quad \text { on }(0, T) \times \Sigma . \\
& \llbracket \boldsymbol{\sigma}(\boldsymbol{v}, p) \cdot \boldsymbol{n} \rrbracket_{\Sigma}:=\left(\mu \nabla \boldsymbol{v}^{f} \cdot \boldsymbol{n}-p^{f} \boldsymbol{n}\right)_{\mid \Sigma}+p_{\mid \Sigma}^{p} \boldsymbol{n}=\frac{\mu}{\sqrt{K}} \boldsymbol{\beta} \overline{\boldsymbol{v}}_{\Sigma}^{\star}
\end{aligned}\right.
\end{aligned}
$$


where the function $\boldsymbol{v}_{\Sigma}^{\star}$ is precised in the sequel and :

$$
\left\{\begin{aligned}
\boldsymbol{\sigma}(\boldsymbol{v}, p)^{f} \cdot \boldsymbol{n}_{\mid \Sigma} & :=\left(\mu \nabla \boldsymbol{v}^{f} \cdot \boldsymbol{n}-p^{f} \boldsymbol{n}\right)_{\mid \Sigma}, \quad \text { and } \quad \boldsymbol{\sigma}(\boldsymbol{v}, p)^{p} \cdot \boldsymbol{n}_{\mid \Sigma}:=-p_{\mid \Sigma}^{p} \boldsymbol{n}, \\
\llbracket \boldsymbol{v} \rrbracket_{\Sigma}^{\star} & :=\boldsymbol{v}_{\mid \Sigma}^{f}-\boldsymbol{v}_{\Sigma}^{\star}, \quad \text { and } \quad \overline{\boldsymbol{v}}_{\Sigma}^{\star}:=\frac{1}{2}\left(\boldsymbol{v}_{\mid \Sigma}^{f}+\boldsymbol{v}_{\Sigma}^{\star}\right) \\
\boldsymbol{v}_{\Sigma}^{\star} \cdot \boldsymbol{n} & =\boldsymbol{v}^{p} \cdot \boldsymbol{n}_{\mid \Sigma}=\boldsymbol{v}^{f} \cdot \boldsymbol{n}_{\mid \Sigma} .
\end{aligned}\right.
$$

Let us define the Hilbert space :

$$
\boldsymbol{U}:=\left\{\boldsymbol{u} \in L^{2}(\Omega)^{d} ; \nabla \cdot \boldsymbol{u}=0 \text { in } \Omega ; \boldsymbol{u}^{f} \in H^{1}\left(\Omega_{f}\right)^{d} ; \llbracket \boldsymbol{u} \cdot \boldsymbol{n} \rrbracket_{\Sigma}=0 ; \boldsymbol{u}_{\mid \Gamma}^{f}=0\right\},
$$

equipped with the natural inner product and associated hilbertian norm defined by :

$$
\|\boldsymbol{u}\|_{\boldsymbol{U}}^{2}:=\|\boldsymbol{u}\|_{0, \Omega}^{2}+\left\|\nabla \boldsymbol{u}^{f}\right\|_{0, \Omega_{f}}^{2}, \quad \forall \boldsymbol{u} \in \boldsymbol{U}
$$

The first condition $\llbracket \boldsymbol{v} \cdot \boldsymbol{n} \rrbracket_{\Sigma}=0$ in Eq. (34) remains included in the solution space $\boldsymbol{U}$ using Proposition 2.2. A function $\boldsymbol{u} \in \boldsymbol{U}$ also satisfies using the Stokes theorem or Green formula: $\int_{\Sigma} \boldsymbol{u} \cdot \boldsymbol{n} \mathrm{d} s=\int_{\Omega_{f}} \boldsymbol{\nabla} \cdot \boldsymbol{u} \mathrm{d} x=0$.

Let us consider the functional setting below identifying the dual space $\boldsymbol{H}^{\prime}$ to $\boldsymbol{H}$ :

$$
\boldsymbol{W} \hookrightarrow \boldsymbol{U} \hookrightarrow \boldsymbol{H} \simeq \boldsymbol{H}^{\prime} \hookrightarrow \boldsymbol{U}^{\prime} \hookrightarrow \boldsymbol{W}^{\prime},
$$

and observe that the continuous imbedding $\boldsymbol{U} \hookrightarrow \boldsymbol{H}$ is dense since we have $\boldsymbol{V} \hookrightarrow \boldsymbol{W} \hookrightarrow \boldsymbol{U} \hookrightarrow \boldsymbol{H}$ and $\boldsymbol{V}$ is dense in $\boldsymbol{H}$, e.g. [19, Sect. IV.3.3].

Using the previous results, we propose the following weak formulation for the Stokes/Darcy problem (33-34) with $\boldsymbol{f} \in L^{2}\left(0, T ; L^{2}(\Omega)^{d}\right)$ using divergence-free test functions in $\boldsymbol{W}$.

Find $\left(\boldsymbol{v}, \boldsymbol{v}_{\Sigma}^{\star}\right)$ such that $\boldsymbol{v} \in \mathcal{C}([0, T] ; \boldsymbol{H}) \cap L^{2}(0, T ; \boldsymbol{U})$ with $\boldsymbol{v}^{\prime}:=\frac{d \boldsymbol{v}}{d t} \in L^{2}(0, T ; \boldsymbol{H})$ and $\boldsymbol{v}(t=0)=\boldsymbol{v}_{0}$ in $\boldsymbol{W}$, and $\boldsymbol{v}_{\Sigma}^{\star} \in L^{2}\left(0, T ; L^{2}(\Sigma)^{d}\right)$ such that $\boldsymbol{v}_{\Sigma}^{\star} \cdot \boldsymbol{n}=\boldsymbol{v}^{p} \cdot \boldsymbol{n}_{\mid \Sigma}=\boldsymbol{v}^{f} \cdot \boldsymbol{n}_{\mid \Sigma}$, that satisfy for all $\boldsymbol{\varphi} \in \boldsymbol{W}$ :

$$
\begin{aligned}
\rho \int_{\Omega} \frac{d \boldsymbol{v}}{d t} \cdot \boldsymbol{\varphi} \mathrm{d} x+\mu \int_{\Omega_{f}} \nabla \boldsymbol{v}: \nabla \boldsymbol{\varphi} \mathrm{d} x & +\mu \int_{\Omega_{p}}\left(\boldsymbol{K}^{-1} \boldsymbol{v}\right) \cdot \boldsymbol{\varphi} \mathrm{d} x \\
& +\frac{\mu}{\sqrt{K}} \int_{\Sigma} \alpha \llbracket \boldsymbol{v} \rrbracket_{\Sigma}^{\star} \cdot \llbracket \boldsymbol{\varphi} \rrbracket_{\Sigma} \mathrm{d} s+\frac{\mu}{\sqrt{K}} \int_{\Sigma}\left(\boldsymbol{\beta} \overline{\boldsymbol{v}}_{\Sigma}^{\star}\right) \cdot \overline{\boldsymbol{\varphi}}_{\Sigma} \mathrm{d} s=\rho \int_{\Omega} \boldsymbol{f} \cdot \boldsymbol{\varphi} \mathrm{d} x, \\
& \text { for a.e. } t \in] 0, T[\quad \forall \boldsymbol{\varphi} \in \boldsymbol{W} .
\end{aligned}
$$

Then, we prove the following main result of solvability in this Section 3 .

\section{Theorem 3.1 (Global well-posedness in time of the Stokes/Darcy problem).}

Let $\boldsymbol{v}_{0} \in \boldsymbol{W}$ in addition to the basic assumptions on the data $\boldsymbol{f}, \boldsymbol{K}, \alpha, \boldsymbol{\beta}$. Then, there exists a solution $\left(\boldsymbol{v}, \boldsymbol{v}_{\Sigma}^{\star}, p\right)$ to the problem (33-34) and verifying the weak form (35) such that $\boldsymbol{v} \in \mathcal{C}([0, T] ; \boldsymbol{H}) \cap L^{2}(0, T ; \boldsymbol{U})$ with $\boldsymbol{v}^{\prime} \in L^{2}(0, T ; \boldsymbol{H}), \boldsymbol{v}_{\Sigma}^{\star} \in L^{2}\left(0, T ; L^{2}(\Sigma)^{d}\right)$ depends linearly on $\boldsymbol{v}$, with $\boldsymbol{v}_{\Sigma}^{\star} \cdot \boldsymbol{n}=\boldsymbol{v}^{p} \cdot \boldsymbol{n}_{\mid \Sigma}=\boldsymbol{v}^{f} \cdot \boldsymbol{n}_{\mid \Sigma}$ in $L^{2}\left(0, T ; H^{1 / 2}(\Sigma)\right)$, and $p \in L^{2}\left(0, T ; L^{2}(\Omega)\right)$ with $p^{p} \in L^{2}\left(0, T ; H^{1}\left(\Omega_{p}\right)\right)$.

Moreover, for any given solution $\boldsymbol{v} \in E:=\left\{\boldsymbol{u} \in L^{2}(0, T ; \boldsymbol{U}) ; \boldsymbol{u}^{\prime} \in L^{2}(0, T ; \boldsymbol{H})\right\}$, the function $\boldsymbol{v}_{\Sigma}^{\star}$ and pressure $p$ are unique and characterized as follows.

i) The operator $\mathcal{T}: E \longrightarrow L^{2}\left(0, T ; L^{2}(\Sigma)^{d}\right)$ which maps $\boldsymbol{v} \longmapsto \boldsymbol{v}_{\Sigma}^{\star}$ such that $\left(\boldsymbol{v}, \boldsymbol{v}_{\Sigma}^{\star}\right)$ satisfies (35) with $\boldsymbol{v}_{\Sigma}^{\star} \cdot \boldsymbol{n}=\boldsymbol{v}^{p} \cdot \boldsymbol{n}_{\mid \Sigma}=\boldsymbol{v}^{f} \cdot \boldsymbol{n}_{\mid \Sigma} \in L^{2}\left(0, T ; H^{1 / 2}(\Sigma)\right)$, is linear and injective. 
ii) As soon as the tangential trace $\boldsymbol{v}^{p} \wedge \boldsymbol{n}_{\mid \Sigma}$ of the Darcy velocity can be defined at least in a weak sense, then we have: $\boldsymbol{v}_{\Sigma}^{\star}=\boldsymbol{v}_{\mid \Sigma}^{p} \in L^{2}\left(0, T ; L^{2}(\Sigma)^{d}\right)$, and $(\boldsymbol{v}, p)$ is solution to the problem (33-34) verifying (35) with $\llbracket \boldsymbol{v} \rrbracket_{\Sigma}^{\star}=\llbracket \boldsymbol{v} \rrbracket_{\Sigma}$ and $\overline{\boldsymbol{v}}_{\Sigma}^{\star}=\overline{\boldsymbol{v}}_{\Sigma}$.

iii) There exists a function $q \in L^{2}\left(0, T ; L^{2}(\Omega)\right)$ with each restriction $q^{f} \in L^{2}\left(0, T ; L_{0}^{2}\left(\Omega_{f}\right)\right)$ and $q^{p} \in$ $L^{2}\left(0, T ; L_{0}^{2}\left(\Omega_{p}\right) \cap H^{1}\left(\Omega_{p}\right)\right)$ both defined uniquely up to an additive constant, such that the pressure field $p \in L^{2}\left(0, T ; L^{2}(\Omega)\right)$ with $p^{p} \in L^{2}\left(0, T ; H^{1}\left(\Omega_{p}\right)\right)$ is defined uniquely by :

$$
p_{\mid \Omega_{f}}:=q^{f}+\frac{\Pi_{0}}{2}, \quad p_{\mid \Omega_{p}}:=q^{p}-\frac{\Pi_{0}}{2}, \quad \text { such that } \llbracket p-q \rrbracket_{\Sigma}=\Pi_{0},
$$

with $\boldsymbol{\sigma}(\boldsymbol{v}, p)^{f} \cdot \boldsymbol{n}_{\mid \Sigma} \in L^{2}\left(0, T ; L^{2}(\Sigma)^{d}\right)$, where the constant quantity $\Pi_{0}$ is defined from $(\boldsymbol{v}, q)$ by :

$$
\Pi_{0}:=\frac{1}{|\Sigma|}\left\langle\llbracket \boldsymbol{\sigma}(\boldsymbol{v}, q) \cdot \boldsymbol{n} \rrbracket_{\Sigma}-\frac{\mu}{\sqrt{K}} \boldsymbol{\beta} \overline{\boldsymbol{v}}_{\Sigma}^{\star}, \boldsymbol{n}\right\rangle_{-1 / 2, \Sigma} .
$$

Furthermore, if the Darcy velocity verifies $\boldsymbol{v}^{p} \in L^{2}\left(0, T ; H^{1}\left(\Omega_{p}\right)^{d}\right)$, then $\boldsymbol{v}_{\Sigma}^{\star}=\boldsymbol{v}_{\mid \Sigma}^{p} \in L^{2}\left(0, T ; H^{1 / 2}(\Sigma)^{d}\right)$, and the solution $(\boldsymbol{v}, p)$ to the problem (33-34) with $\llbracket \boldsymbol{v} \rrbracket_{\Sigma}^{\star}=\llbracket \boldsymbol{v} \rrbracket_{\Sigma}$ and $\overline{\boldsymbol{v}}_{\Sigma}^{\star}=\overline{\boldsymbol{v}}_{\Sigma}$ is unique and satisfies the energy equality below:

$$
\begin{aligned}
\frac{\rho}{2}\|\boldsymbol{v}(t)\|_{0, \Omega}^{2}+\mu \int_{0}^{t} \int_{\Omega_{f}}|\nabla \boldsymbol{v}|^{2} \mathrm{~d} x \mathrm{~d} \tau & +\mu \int_{0}^{t} \int_{\Omega_{p}}\left(K^{-1} \boldsymbol{v}\right) \cdot \boldsymbol{v} \mathrm{d} x \mathrm{~d} \tau \\
& +\frac{\mu}{\sqrt{K}} \int_{0}^{t} \int_{\Sigma} \alpha \llbracket \boldsymbol{v} \rrbracket_{\Sigma} \cdot \llbracket \boldsymbol{v} \rrbracket_{\Sigma} \mathrm{d} s \mathrm{~d} \tau+\frac{\mu}{\sqrt{K}} \int_{0}^{t} \int_{\Sigma}\left(\boldsymbol{\beta} \overline{\boldsymbol{v}}_{\Sigma}\right) \cdot \overline{\boldsymbol{v}}_{\Sigma} \mathrm{d} s \mathrm{~d} \tau \\
& =\frac{\rho}{2}\left\|\boldsymbol{v}_{0}\right\|_{0, \Omega}^{2}+\rho \int_{0}^{t} \int_{\Omega} \boldsymbol{f} \cdot \boldsymbol{v} \mathrm{d} x \mathrm{~d} \tau, \quad \text { for all } t \in[0, T] .
\end{aligned}
$$

Proof. Summarizing the results shown in Sections 3.1 and 3.2, the existence of the solution $\left(\boldsymbol{v}, \boldsymbol{v}_{\Sigma}^{\star}, p\right)$ to the problem (33-34) and satisfying the weak form (35) is proved such that $\boldsymbol{v} \in L^{2}(0, T ; \boldsymbol{U})$ with $\boldsymbol{v}^{\prime} \in L^{2}(0, T ; \boldsymbol{H})$. This ensures that $\boldsymbol{v} \in \mathcal{C}([0, T] ; \boldsymbol{H})$ by applying Lions-Magenes theorem since the continuous imbedding $\boldsymbol{U} \hookrightarrow \boldsymbol{H}$ is dense because $\boldsymbol{V} \hookrightarrow \boldsymbol{U} \hookrightarrow \boldsymbol{H}$ and $\boldsymbol{V}$ is dense in $\boldsymbol{H}$.

We have also shown the existence of $\boldsymbol{v}_{\Sigma}^{\star} \in L^{2}\left(0, T ; L^{2}(\Sigma)^{d}\right)$. Moreover, as soon as the tangential trace $\boldsymbol{v}^{p} \wedge \boldsymbol{n}_{\mid \Sigma}$ of the Darcy velocity can be defined at least in a weak sense, e.g. in $H^{-1 / 2}(\Sigma)^{d}$ when $\boldsymbol{v}^{p} \in \boldsymbol{H}_{\text {rot }}\left(\Omega_{p}\right)$, then we know from the points $e), f), g), h)$ in Section 3.1 that $\boldsymbol{v}_{\Sigma}^{\star}=\boldsymbol{v}_{\mid \Sigma}^{p} \in L^{2}\left(0, T ; L^{2}(\Sigma)^{d}\right)$ and both $\llbracket \boldsymbol{v} \rrbracket_{\Sigma}, \overline{\boldsymbol{v}}_{\Sigma}$ belong to $L^{2}\left(0, T ; L^{2}(\Sigma)^{d}\right)$ because $\boldsymbol{v}_{\mid \Sigma}^{f} \in L^{2}\left(0, T ; H^{1 / 2}(\Sigma)^{d}\right)$.

Furthermore, the pressure field $p \in L^{2}\left(0, T ; L^{2}(\Omega)\right)$ with $p^{p} \in L^{2}\left(0, T ; H^{1}\left(\Omega_{p}\right)\right)$ defined by (36-37) was also proved to be unique as soon as a velocity solution $\left(\boldsymbol{v}, \boldsymbol{v}_{\Sigma}^{\star}\right)$ to Eq. (35) is given.

Hence, its remains to characterize more precisely the function $\boldsymbol{v}_{\Sigma}^{\star}$ with respect to $\boldsymbol{v}$, and if $\boldsymbol{v}^{p}(t) \in H^{1}\left(\Omega_{p}\right)^{d}$ for a.e. $t \in[0, T]$ and thus $\boldsymbol{v}_{\Sigma}^{\star}(t)=\boldsymbol{v}_{\mid \Sigma}^{p}(t) \in H^{1 / 2}(\Sigma)^{d}$, to prove the energy equality (38) that leads to the uniqueness of the velocity field $\boldsymbol{v}$ as an easy consequence.

\section{a) Characterization of the function $\boldsymbol{v}_{\Sigma}^{\star} \in L^{2}\left(0, T ; L^{2}(\Sigma)^{d}\right)$.}

We have already shown that $\boldsymbol{v}_{\Sigma}^{\star} \in L^{2}\left(0, T ; L^{2}(\Sigma)^{d}\right)$ verifies $\boldsymbol{v}_{\Sigma}^{\star} \cdot \boldsymbol{n}=\boldsymbol{v}^{p} \cdot \boldsymbol{n}_{\mid \Sigma}=\boldsymbol{v}^{f} \cdot \boldsymbol{n}_{\mid \Sigma} \in L^{2}\left(0, T ; H^{1 / 2}(\Sigma)\right)$.

Let us show that $\boldsymbol{v}_{\Sigma}^{\star}$ depends only on the Stokes/Darcy velocity field $\boldsymbol{v}$, i.e. if a solution $\left(\boldsymbol{v}, \boldsymbol{v}_{\Sigma}^{\star}\right)$ to the weak problem (35) is given, then $\boldsymbol{v}_{\Sigma}^{\star}$ associated to $\boldsymbol{v}$ is unique.

Suppose that there exist two functions $\boldsymbol{u}_{\Sigma}^{\star}$ and $\boldsymbol{v}_{\Sigma}^{\star}$ verifying both (35) with to the same field $\boldsymbol{v}$ and the same data, knowing that $\boldsymbol{u}_{\Sigma}^{\star} \cdot \boldsymbol{n}=\boldsymbol{v}_{\Sigma}^{\star} \cdot \boldsymbol{n}=\boldsymbol{v}^{p} \cdot \boldsymbol{n}_{\mid \Sigma}=\boldsymbol{v}^{f} \cdot \boldsymbol{n}_{\mid \Sigma}$. By differencing these two linear equations, we have for 
a.e. $t \in] 0, T[$ :

$$
\frac{\mu}{\sqrt{K}} \int_{\Sigma} \alpha\left(\boldsymbol{u}_{\Sigma}^{\star}-\boldsymbol{v}_{\Sigma}^{\star}\right) \cdot \llbracket \boldsymbol{\varphi} \rrbracket_{\Sigma} \mathrm{d} s+\frac{\mu}{2 \sqrt{K}} \int_{\Sigma}\left(\boldsymbol{\beta}\left(\boldsymbol{u}_{\Sigma}^{\star}-\boldsymbol{v}_{\Sigma}^{\star}\right)\right) \cdot \overline{\boldsymbol{\varphi}}_{\Sigma} \mathrm{d} s=0, \quad \forall \boldsymbol{\varphi} \in \boldsymbol{W} .
$$

In particular, for any test function $\varphi \in \boldsymbol{W}$ such that $\overline{\boldsymbol{\varphi}}_{\Sigma}=0$ and so $\llbracket \varphi \rrbracket_{\Sigma}=-2 \boldsymbol{\varphi}_{\mid \Sigma}^{p}$, we have :

$$
\int_{\Sigma} \alpha\left(\boldsymbol{u}_{\Sigma}^{\star}-\boldsymbol{v}_{\Sigma}^{\star}\right) \cdot \varphi_{\mid \Sigma}^{p} \mathrm{~d} s=0
$$

which means, using the fact that $\Sigma$ is a closed surface, that

$$
\int_{\Sigma} \alpha\left(\boldsymbol{u}_{\Sigma}^{\star}-\boldsymbol{v}_{\Sigma}^{\star}\right) \cdot \boldsymbol{\varphi}_{\mid \Sigma} \mathrm{d} s=0, \quad \forall \boldsymbol{\varphi} \in H^{1}\left(\Omega_{p}\right)^{d} ; \quad \boldsymbol{\nabla} \cdot \boldsymbol{\varphi}=0 \text { in } \Omega_{p} .
$$

Applying now Lemma 2.4 in $\Omega_{p}$, for any $\boldsymbol{\psi} \in H^{1 / 2}(\Sigma)^{d}$, there exists $\varphi \in H^{1}\left(\Omega_{p}\right)^{d}$ with $\boldsymbol{\nabla} \cdot \boldsymbol{\varphi}=0$ in $\Omega_{p}$ such that

$$
\boldsymbol{\psi}=\boldsymbol{\varphi}_{\mid \Sigma}+\frac{1}{|\Sigma|}\left(\int_{\Sigma} \boldsymbol{\psi} \cdot \boldsymbol{n} \mathrm{d} s\right) \boldsymbol{n}
$$

Using this in the previous equation with $\left(\boldsymbol{u}_{\Sigma}^{\star}-\boldsymbol{v}_{\Sigma}^{\star}\right) \cdot \boldsymbol{n}=0$, it yields

$$
\begin{aligned}
\int_{\Sigma} \alpha\left(\boldsymbol{u}_{\Sigma}^{\star}-\boldsymbol{v}_{\Sigma}^{\star}\right) \cdot \boldsymbol{\psi} \mathrm{d} s & =\frac{1}{|\Sigma|}\left(\int_{\Sigma} \boldsymbol{\psi} \cdot \boldsymbol{n} \mathrm{d} s\right) \int_{\Sigma} \alpha\left(\boldsymbol{u}_{\Sigma}^{\star}-\boldsymbol{v}_{\Sigma}^{\star}\right) \cdot \boldsymbol{n} \mathrm{d} s \\
& =0, \quad \forall \boldsymbol{\psi} \in H^{1 / 2}(\Sigma)^{d}
\end{aligned}
$$

Thus we get the desired result: $\boldsymbol{u}_{\Sigma}^{\star}=\boldsymbol{v}_{\Sigma}^{\star}$.

This allows us to define the mapping :

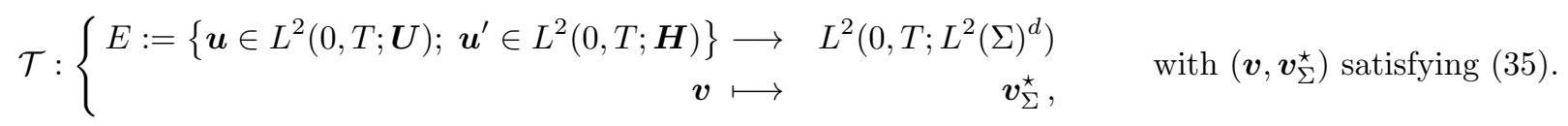

By proceeding as above, it is clear that the application $\mathcal{T}$ is linear.

Let us show that the operator $\mathcal{T}$ is injective by considering $\boldsymbol{v} \in E$ satisfying (35) with $\mathcal{T}(\boldsymbol{v})=\boldsymbol{v}_{\Sigma}^{\star}=0$ and thus $\boldsymbol{v} \cdot \boldsymbol{n}_{\mid \Sigma}=0$, together with null data $\boldsymbol{f}=0$ and $\boldsymbol{v}_{0}=0$. Then $\boldsymbol{v}$ verifies from (35):

$$
\begin{aligned}
\rho \int_{\Omega} \frac{d \boldsymbol{v}}{d t} \cdot \boldsymbol{\varphi} \mathrm{d} x+\mu \int_{\Omega_{f}} \nabla \boldsymbol{v}: \nabla \boldsymbol{\varphi} \mathrm{d} x & +\mu \int_{\Omega_{p}}\left(\boldsymbol{K}^{-1} \boldsymbol{v}\right) \cdot \boldsymbol{\varphi} \mathrm{d} x \\
& +\frac{\mu}{\sqrt{K}} \int_{\Sigma} \alpha \boldsymbol{v}_{\mid \Sigma}^{f} \cdot \llbracket \boldsymbol{\varphi} \rrbracket_{\Sigma} \mathrm{d} s+\frac{\mu}{2 \sqrt{K}} \int_{\Sigma}\left(\boldsymbol{\beta} \boldsymbol{v}_{\mid \Sigma}^{f}\right) \cdot \overline{\boldsymbol{\varphi}}_{\Sigma} \mathrm{d} s=0, \\
& \text { for a.e. } t \in] 0, T[\quad \forall \boldsymbol{\varphi} \in \boldsymbol{W} .
\end{aligned}
$$

In particular, by taking $\boldsymbol{\varphi} \in \boldsymbol{W}$ such that $\varphi^{f}=\boldsymbol{v}^{f}(t) \in H_{0 \Gamma}^{1}\left(\Omega_{f}\right)$ with $\boldsymbol{\nabla} \cdot \boldsymbol{v}^{f}=0$ and $\boldsymbol{\varphi}^{p}=0$, we have for a.e. $t \in] 0, T[:$

$$
\frac{\rho}{2} \frac{d}{d t} \int_{\Omega_{f}}\left|\boldsymbol{v}^{f}\right|^{2} \mathrm{~d} x+\mu \int_{\Omega_{f}}\left|\nabla \boldsymbol{v}^{f}\right|^{2} \mathrm{~d} x+\frac{\mu}{\sqrt{K}} \int_{\Sigma} \alpha \boldsymbol{v}_{\mid \Sigma}^{f} \cdot \boldsymbol{v}_{\mid \Sigma}^{f} \mathrm{~d} s+\frac{\mu}{4 \sqrt{K}} \int_{\Sigma}\left(\boldsymbol{\beta} \boldsymbol{v}_{\mid \Sigma}^{f}\right) \cdot \boldsymbol{v}_{\mid \Sigma}^{f} \mathrm{~d} s=0 .
$$


By integrating in time over $(0, t)$ for all $t \in[0, T]$ with the initial condition $\boldsymbol{v}_{0}=0$, it yields: $\boldsymbol{v}^{f}=0$. Now $\boldsymbol{v}^{p}$ verifies :

$$
\left.\rho \int_{\Omega_{p}} \frac{d \boldsymbol{v}}{d t} \cdot \boldsymbol{\varphi} \mathrm{d} x+\mu \int_{\Omega_{p}}\left(\boldsymbol{K}^{-1} \boldsymbol{v}\right) \cdot \boldsymbol{\varphi} \mathrm{d} x=0, \quad \text { for a.e. } t \in\right] 0, T[; \quad \forall \boldsymbol{\varphi} \in \boldsymbol{W} .
$$

Here, it is not allowed to take directly $\varphi=\boldsymbol{v}^{p}(t)$ because $\boldsymbol{v}^{p}(t) \in L^{2}\left(\Omega_{p}\right)^{d}$ does not belong a priori to $H^{1}\left(\Omega_{p}\right)^{d}$. Nevertheless, by interpreting the above problem with any $\varphi \in H_{0}^{1}\left(\Omega_{p}\right)^{d}$ such that $\boldsymbol{\nabla} \cdot \boldsymbol{\varphi}=0$ in $\Omega_{p}$ and applying De Rham's theorem, it yields that $\boldsymbol{v}^{p}$ is solution of the Darcy problem below :

$$
\left\{\begin{aligned}
\rho \partial_{t} \boldsymbol{v}^{p}+\mu \boldsymbol{K}^{-1} \boldsymbol{v}^{p}+\nabla p & =0, & & \text { in }(0, T) \times \Omega_{p}, \\
\boldsymbol{\nabla} \cdot \boldsymbol{v}^{p} & =0, & & \text { in }(0, T) \times \Omega_{p}, \\
\boldsymbol{v}^{p} \cdot \boldsymbol{n}_{\mid \Sigma} & =0, & & \text { on }(0, T) \times \partial \Omega_{p}, \\
\boldsymbol{v}^{p}(t=0) & =0, & & \text { in } \Omega_{p} .
\end{aligned}\right.
$$

Then it is clear solving this problem that the unique solution is $\boldsymbol{v}^{p}=0$. Hence, we have finally $\boldsymbol{v}=0$ which shows the injectivity of $\mathcal{T}$.

\section{b) Energy equality and uniqueness.}

Here, we make the hypothesis of sufficient regularity of the Darcy problem in $\Omega_{p}$. Let us assume that the filtration velocity verifies $\boldsymbol{v}^{p} \in L^{2}\left(0, T ; H^{1}\left(\Omega_{p}\right)^{d}\right)$. Then, we have from what preceeds $\boldsymbol{v}_{\Sigma}^{\star}=\boldsymbol{v}_{\mid \Sigma}^{p} \in L^{2}\left(0, T ; H^{1 / 2}(\Sigma)^{d}\right)$, and $\llbracket \boldsymbol{v} \rrbracket_{\Sigma}^{\star}=\llbracket \boldsymbol{v} \rrbracket_{\Sigma}, \overline{\boldsymbol{v}}_{\Sigma}^{\star}=\overline{\boldsymbol{v}}_{\Sigma}$.

In that case, any solution $\boldsymbol{v} \in E$ to (35) satisfies $\boldsymbol{v} \in L^{2}(0, T ; \boldsymbol{W})$, and (35) becomes :

$$
\begin{aligned}
\rho \int_{\Omega} \frac{d \boldsymbol{v}}{d t} \cdot \boldsymbol{\varphi} \mathrm{d} x+\mu \int_{\Omega_{f}} \nabla \boldsymbol{v}: \nabla \boldsymbol{\varphi} \mathrm{d} x & +\mu \int_{\Omega_{p}}\left(\boldsymbol{K}^{-1} \boldsymbol{v}\right) \cdot \boldsymbol{\varphi} \mathrm{d} x \\
& +\frac{\mu}{\sqrt{K}} \int_{\Sigma} \alpha \llbracket \boldsymbol{v} \rrbracket_{\Sigma} \cdot \llbracket \boldsymbol{\varphi} \rrbracket_{\Sigma} \mathrm{d} s+\frac{\mu}{\sqrt{K}} \int_{\Sigma}\left(\boldsymbol{\beta} \overline{\boldsymbol{v}}_{\Sigma}\right) \cdot \overline{\boldsymbol{\varphi}}_{\Sigma} \mathrm{d} s=\rho \int_{\Omega} \boldsymbol{f} \cdot \boldsymbol{\varphi} \mathrm{d} x, \\
& \text { for a.e. } t \in] 0, T[\quad \forall \boldsymbol{\varphi} \in \boldsymbol{W} .
\end{aligned}
$$

It is now allowed to take $\boldsymbol{\varphi}=\boldsymbol{v}(t) \in \boldsymbol{W}$. Then, integrating in time over $(0, t)$ for all $t \in[0, T]$ using the initial condition $\boldsymbol{v}(t=0)=\boldsymbol{v}_{0}$, the energy equality (38) is easily obtained.

Moreover, the uniqueness of velocity solution $\boldsymbol{v}$ becomes an immediate consequence since (38) with null data $\boldsymbol{f}=0$ and $\boldsymbol{v}_{0}$ implies $\boldsymbol{v}=0$. Then, the uniqueness of the pressure field $p$ follows from what preceeds, which ends the proof.

With the weak form (35), since the space $\boldsymbol{W}$ is not dense in $\boldsymbol{U}$, it does not seem possible to prove the uniqueness of the velocity field $\boldsymbol{v}$ in the general case with no extra regularity hypothesis. Hence, despite the linearity of this problem, the question of velocity uniqueness remains open in Theorem 3.1 although it was claimed too fast in [8] to hold for the corresponding steady Stokes/Darcy problem. However, we think that it is probably not true.

Remark 3.2 (On the convergence from Stokes/Brinkman to Stokes/Darcy by vanishing viscosity). When the solution to the limit Stokes/Darcy problem (33-34) is unique with Theorem 3.1, one can conclude by Cantor's lemma on the convergence of a sequence by double extraction, that the whole sequence $\left(\boldsymbol{v}_{\varepsilon}\right)_{\varepsilon>0}$ in the Stokes/Brinkman problem (27-28), and not only a subsequence, converges to the solution $\boldsymbol{v}$ of (33-34) in the sense defined in Section 3.1.

Moreover, using Aubin-Lions compactness theorem [49, Chap. 1] since the continuous imbedding $H^{1}\left(\Omega_{f}\right)^{d} \hookrightarrow$ $L^{2}\left(\Omega_{f}\right)^{d}$ is compact by Rellich's theorem, the sequence $\left(\boldsymbol{v}_{\varepsilon}^{f}\right)_{\varepsilon>0}$ converges strongly to $\boldsymbol{v}^{f}$ in $L^{2}\left(0, T ; L^{2}\left(\Omega_{f}\right)^{d}\right)$. 
In [10], the BKW method of asymptotic expansion is applied to calculate the resulting viscous boundary layer at the interface $\Sigma$ and located inside $\Omega_{p}$ which is involved by the singular perturbation of vanishing viscosity in $\Omega_{p}$ and then, the asymptotic expansion is proved to converge. This analysis was made in the steady case for a slightly modified set of jump interface conditions, but requires a smooth solution.

\section{Remark 3.3 (On the "trace" function $v_{\Sigma}^{\star}$ ).}

We have shown in Theorem 3.1 that for any velocity solution $\boldsymbol{v}$ to the Stokes/Darcy problem, the function $\boldsymbol{v}_{\Sigma}^{\star}$ is unique depending linearly on $\boldsymbol{v}$. But, in the general case with standard regularity of the problem, it is not possible to claim that the function $\boldsymbol{v}_{\Sigma}^{\star} \in L^{2}\left(0, T ; L^{2}(\Sigma)^{d}\right)$ is the trace on $\Sigma$ of the filtration velocity $\boldsymbol{v}^{p}$ in the porous medium. It is only the case for the normal trace $\boldsymbol{v}_{\Sigma}^{\star} \cdot \boldsymbol{n}=\boldsymbol{v}^{p} \cdot \boldsymbol{n}_{\mid \Sigma}=\boldsymbol{v}^{f} \cdot \boldsymbol{n}_{\mid \Sigma}$. Indeed, $\boldsymbol{v}^{p}(t)$ only belongs to $\boldsymbol{H}_{\text {div }}\left(\Omega_{p}\right)$ and has no tangential trace on $\Sigma$.

However, as soon as the problem is more regular such that a tangential trace of $\boldsymbol{v}^{p}(t)$ can be defined, at least in a weak sense, then we actually get: $\boldsymbol{v}_{\mid \Sigma}^{p}(t)=\boldsymbol{v}_{\Sigma}^{\star}(t) \in L^{2}(\Sigma)^{d}$ for a.e. $t \in[0, T]$. It occurs when the Darcy velocity $\boldsymbol{v}^{p}(t)$ in $\Omega_{p}$ belongs to $\boldsymbol{H}_{\text {rot }}\left(\Omega_{p}\right)$. For instance, it is the case for the steady Darcy equation with a constant permeability tensor $\boldsymbol{K}$ and a body force $\boldsymbol{f}^{p} \in \boldsymbol{H}_{\text {rot }}\left(\Omega_{p}\right)$, e.g. for the constant gravity force, since we have with $\nabla \times(\nabla p)=0$ :

$$
\boldsymbol{\nabla} \times \boldsymbol{v}^{p}=\frac{\rho}{\mu} \boldsymbol{K} \nabla \times \boldsymbol{f}^{p} \in L^{2}\left(\Omega_{p}\right)^{d} .
$$

Then $\boldsymbol{v}^{p}(t)$ admits a tangential trace $\boldsymbol{v}^{p} \wedge \boldsymbol{n}_{\mid \Sigma} \in H^{-1 / 2}(\Sigma)^{d}$ [35, Theorem 2.11]. In that case, Theorem 3.1 holds and we have $\boldsymbol{v}^{p} \in L^{2}\left(0, T ; \boldsymbol{H}_{\text {div }}\left(\Omega_{p}\right) \cap \boldsymbol{H}_{\text {rot }}\left(\Omega_{p}\right)\right)$ and $\boldsymbol{v}_{\Sigma}^{\star}=\boldsymbol{v}_{\mid \Sigma}^{p}$ belongs to $L^{2}\left(0, T ; L^{2}(\Sigma)^{d}\right)$.

The analysis of regularity of the general Stokes/Darcy problem would deserve a study in itself and lies beyond the scope of the present paper; see Corollary 3.4 further for a particular case.

In many practical situations, e.g. for the study of geothermal/geophysics systems, the term of velocity time derivative can be neglected in the Darcy equation. Indeed, by choosing a suitable dimensioning to get the dimensionless Darcy equation [25], the coefficient of the time derivative includes the Darcy number Da := $K / L^{2}=\mathcal{O}\left(d^{2} / L^{2}\right) \ll 1$, as discussed in Remark 1.1 and Appendix A. Then, the flow in the permeable medium is quasi-stationary driven by the transient free fluid flow and the governing equations read in the constant gravity field $\boldsymbol{g}$ :

$$
\left\{\begin{aligned}
\nabla \cdot \boldsymbol{v} & =0 & & \text { in }(0, T) \times \Omega, \\
\rho \partial_{t} \boldsymbol{v}-\mu \Delta \boldsymbol{v}+\nabla p & =\rho \boldsymbol{f} & & \text { in }(0, T) \times \Omega_{f}, \\
\mu \boldsymbol{K}^{-1} \boldsymbol{v}+\nabla p & =\rho \boldsymbol{g} & & \text { in }(0, T) \times \Omega_{p}, \\
\boldsymbol{v} & =0 & & \text { on }(0, T) \times \Gamma, \\
\boldsymbol{v}(t=0) & =\boldsymbol{v}_{0} \in \boldsymbol{W} & & \text { in } \Omega_{f} .
\end{aligned}\right.
$$

If now the porous medium is homogeneous and isotropic, the permeability tensor $\boldsymbol{K}:=K \boldsymbol{I}$ is constant. In that case, the steady Darcy equation reduces to solve the Neumann-Poisson problem below for the pressure :

$$
\left\{\begin{aligned}
\Delta p & =0 & & \text { in }(0, T) \times \Omega_{p}, \\
\nabla p \cdot \boldsymbol{n}_{\mid \Sigma} & =\rho \boldsymbol{g} \cdot \boldsymbol{n}_{\mid \Sigma}-\frac{\mu}{K} \boldsymbol{v}^{f} \cdot \boldsymbol{n}_{\mid \Sigma} & & \text { on }(0, T) \times \Sigma,
\end{aligned}\right.
$$

that verifies the compatibility condition: $\int_{\Sigma} \nabla p \cdot \boldsymbol{n} \mathrm{d} s=0$, because $\int_{\Sigma} \boldsymbol{g} \cdot \boldsymbol{n} \mathrm{d} s=0$ and $\int_{\Sigma} \boldsymbol{v} \cdot \boldsymbol{n} \mathrm{d} s=0$.

\section{Corollary 3.4 (Well-posedness for Stokes/steady Darcy problem).}

In addition to the hypotheses of Theorem 3.1, let us assume that the surface $\Sigma$ is of class $\mathcal{C}^{1,1}$ or that the domain $\Omega_{p}$ is a bounded convex polyhedron in $\mathbb{R}^{3}$. Let us consider the unsteady Stokes flow and the steady Darcy flow (39) with an isotropic constant permeability $\boldsymbol{K}:=K \boldsymbol{I}$, coupled with the jump interface conditions (34). 
Then, Theorem 3.1 holds with a unique solution $(\boldsymbol{v}, p)$ such that $p^{p} \in L^{2}\left(0, T ; H^{2}\left(\Omega^{p}\right)\right), \boldsymbol{v}^{p} \in L^{2}\left(0, T ; H^{1}\left(\Omega^{p}\right)^{d}\right)$ and $\boldsymbol{v}_{\Sigma}^{\star}=\boldsymbol{v}_{\mid \Sigma}^{p}$ belongs to $L^{2}\left(0, T ; H^{1 / 2}(\Sigma)^{d}\right)$.

Proof. The domain $\Omega_{p}$ and the data are sufficiently smooth so that the Neumann-Poisson problem (40) gets the $H^{2}$-elliptic regularity; see $[1,34,40]$. Since $\boldsymbol{n} \in H^{1 / 2}(\Sigma)^{d}$ for a Lipschitz surface $\Sigma$, for any velocity field $\boldsymbol{v}^{f} \in L^{2}\left(0, T ; H_{0, \Gamma}^{1}\left(\Omega_{f}\right)^{d}\right.$ with $\partial_{t} \boldsymbol{v}^{f} \in L^{2}\left(0, T ; L^{2}\left(\Omega_{f}\right)^{d}\right)$ verifying the unsteady Stokes equation in $\Omega_{f}$, we have $\boldsymbol{v} \cdot \boldsymbol{n}_{\mid \Sigma}=\boldsymbol{v}^{f} \cdot \boldsymbol{n}_{\mid \Sigma} \in L^{2}\left(0, T ; H^{1 / 2}(\Sigma)\right)$, and thus there exists a unique pressure solution $p^{p}$ of problem (40) which belongs to $L^{2}\left(0, T ; H^{2}\left(\Omega_{p}\right) \cap L_{0}^{2}\left(\Omega_{p}\right)\right)$. Then, we have $\boldsymbol{v}^{p} \in L^{2}\left(0, T ; H^{1}\left(\Omega_{p}\right)^{d}\right)$ for the filtration velocity with Darcy's law in Eq. (39) and thus $\boldsymbol{v}_{\Sigma}^{\star}=\boldsymbol{v}_{\mid \Sigma}^{p} \in L^{2}\left(0, T ; H^{1 / 2}(\Sigma)^{d}\right)$.

Hence from (35), the weak form of velocity problem (39) becomes with $\boldsymbol{v}_{0} \in \boldsymbol{W}$ :

find $\boldsymbol{v} \in L^{2}(0, T ; \boldsymbol{W})$ with $\frac{d \boldsymbol{v}^{f}}{d t} \in L^{2}\left(0, T ; L^{2}\left(\Omega_{f}\right)^{d}\right)$ and $\boldsymbol{v}^{f}(t=0)=\boldsymbol{v}_{0}^{f}$ such that :

$$
\begin{aligned}
\rho \int_{\Omega_{f}} \frac{d \boldsymbol{v}}{d t} \cdot \boldsymbol{\varphi} \mathrm{d} x+\mu \int_{\Omega_{f}} \nabla \boldsymbol{v}: \nabla \boldsymbol{\varphi} \mathrm{d} x & +\mu \int_{\Omega_{p}}\left(\boldsymbol{K}^{-1} \boldsymbol{v}\right) \cdot \boldsymbol{\varphi} \mathrm{d} x \\
& +\frac{\mu}{\sqrt{K}} \int_{\Sigma} \alpha \llbracket \boldsymbol{v} \rrbracket_{\Sigma} \cdot \llbracket \boldsymbol{\varphi} \rrbracket_{\Sigma} \mathrm{d} s+\frac{\mu}{\sqrt{K}} \int_{\Sigma}\left(\boldsymbol{\beta} \overline{\boldsymbol{v}}_{\Sigma}\right) \cdot \overline{\boldsymbol{\varphi}}_{\Sigma} \mathrm{d} s=\rho \int_{\Omega} \boldsymbol{f} \cdot \boldsymbol{\varphi} \mathrm{d} x,
\end{aligned}
$$

for a.e. $t \in] 0, T[\quad \forall \varphi \in \boldsymbol{W}$.

Then, the uniqueness of velocity $\boldsymbol{v}$ verifying (41), recovering the momentum equations and the jump interface conditions (34) by adjusting the pressure $p$, will follow the proof of Theorem 3.1.

However, the existence of solution to (41) is no more a direct consequence of J.L. Lions' abstract setting, as in Theorem 2.5 for the unsteady Stokes/Brinkman problem, since the velocity time derivative is no more explicitly controlled in $\Omega_{p}$. Let us show that $\partial_{t} \boldsymbol{v}^{p}$ in $\Omega_{p}$ is actually controlled by $\partial_{t} \boldsymbol{v}^{f}$ in $\Omega_{f}$. Indeed from (40), $\partial_{t} p^{p}$ in $\Omega_{p}$ satisfies :

$$
\left\{\begin{aligned}
\Delta \partial_{t} p & =0 & & \text { in }(0, T) \times \Omega_{p}, \\
\nabla \partial_{t} p \cdot \boldsymbol{n}_{\mid \Sigma} & =-\frac{\mu}{K} \partial_{t} \boldsymbol{v}^{f} \cdot \boldsymbol{n}_{\mid \Sigma} & & \text { on }(0, T) \times \Sigma .
\end{aligned}\right.
$$

With $\partial_{t} \boldsymbol{v}^{f} \in L^{2}\left(0, T ; L^{2}\left(\Omega_{f}\right)^{d}\right)$ and $\nabla \cdot \partial_{t} \boldsymbol{v}^{f}=0$, we have $\partial_{t} \boldsymbol{v}^{f} \cdot \boldsymbol{n}_{\mid \Sigma} \in L^{2}\left(0, T ; H^{-1 / 2}(\Sigma)\right)$. Thus, the problem (42) admits a unique solution $\partial_{t} p^{p} \in L^{2}\left(0, T ; H^{1}\left(\Omega_{p}\right) \cap L_{0}^{2}\left(\Omega_{p}\right)\right)$ that satisfies the bound :

$$
\begin{aligned}
\left\|\nabla \partial_{t} p^{p}\right\|_{L^{2}\left(0, T ; L^{2}\left(\Omega_{p}\right)^{d}\right)} & \leq c\left(\Omega_{p}\right) \frac{\mu}{K}\left\|\partial_{t} \boldsymbol{v}^{f} \cdot \boldsymbol{n}_{\mid \Sigma}\right\|_{L^{2}\left(0, T ; H^{-1 / 2}(\Sigma)\right)} \\
& \leq c\left(\Omega_{f}, \Omega_{p}\right) \frac{\mu}{K}\left\|\partial_{t} \boldsymbol{v}^{f}\right\|_{L^{2}\left(0, T ; L^{2}\left(\Omega_{f}\right)^{d}\right)} .
\end{aligned}
$$

Hence, we get from the Darcy law in (39) and the previous bound :

$$
\left\|\partial_{t} \boldsymbol{v}^{p}\right\|_{L^{2}\left(0, T ; L^{2}\left(\Omega_{p}\right)^{d}\right)}=\frac{K}{\mu}\left\|\nabla \partial_{t} p^{p}\right\|_{L^{2}\left(0, T ; L^{2}\left(\Omega_{p}\right)^{d}\right)} \leq c\left(\Omega_{f}, \Omega_{p}\right)\left\|\partial_{t} \boldsymbol{v}^{f}\right\|_{L^{2}\left(0, T ; L^{2}\left(\Omega_{f}\right)^{d}\right)} .
$$

Therefore, a solution of problem (41) satisfies $\boldsymbol{v} \in L^{2}(0, T ; \boldsymbol{W})$ with $\boldsymbol{v}^{\prime} \in L^{2}(0, T ; \boldsymbol{H})$ and thus $\boldsymbol{v} \in \mathcal{C}([0, T] ; \boldsymbol{H})$.

As explained hereafter, the proof of existence can be actually made by passing to the limit when the time step $\delta t$ tends to 0 within a semi-discretization in time of the unsteady Stokes problem in $\Omega_{f}$ coupled to the steady Brinkman equation in $\Omega_{p}$.

For a given time step $0<\delta t \leq T, u^{n}$ denotes a desired approximation of any function $u($.$) at time t_{n}=n \delta t$ for 
all $n \in \mathbb{N}$ such that $(n+1) \delta t \leq T$. More precisely, we have for $\boldsymbol{f} \in L^{2}\left(0, T ; L^{2}(\Omega)^{d}\right)$ :

$$
\begin{aligned}
\boldsymbol{f}^{n+1}:= & \frac{1}{\delta t} \int_{n \delta t}^{(n+1) \delta t} \boldsymbol{f}(t) \mathrm{d} t \in L^{2}(\Omega)^{d}, \\
& \text { such that } \quad \sum_{k=0}^{n} \delta t\left\|\boldsymbol{f}^{k+1}\right\|_{0, \Omega}^{2} \leq \sum_{k=0}^{n} \int_{k \delta t}^{(k+1) \delta t}\|\boldsymbol{f}(t)\|_{0, \Omega}^{2} \mathrm{~d} t \leq \int_{0}^{T}\|\boldsymbol{f}(t)\|_{0, \Omega}^{2} \mathrm{~d} t .
\end{aligned}
$$

We consider the implicit Euler scheme for the unsteady Stokes/steady Brinkman problem with the initial data $\boldsymbol{v}^{0}=\boldsymbol{v}_{0} \in \boldsymbol{W}$ :

$$
\begin{aligned}
\rho \int_{\Omega_{f}} \frac{\boldsymbol{v}^{n+1}-\boldsymbol{v}^{n}}{\delta t} \cdot \boldsymbol{\varphi} \mathrm{d} x & +\mu \int_{\Omega_{f}} \nabla \boldsymbol{v}^{n+1}: \nabla \boldsymbol{\varphi} \mathrm{d} x+\int_{\Omega_{p}} \widetilde{\mu}^{p} \nabla \boldsymbol{v}^{n+1}: \nabla \boldsymbol{\varphi} \mathrm{d} x+\mu \int_{\Omega_{p}}\left(\boldsymbol{K}^{-1} \boldsymbol{v}^{n+1}\right) \cdot \boldsymbol{\varphi} \mathrm{d} x \\
& +\frac{\mu}{\sqrt{K}} \int_{\Sigma} \alpha \llbracket \boldsymbol{v}^{n+1} \rrbracket_{\Sigma} \cdot \llbracket \boldsymbol{\varphi} \rrbracket_{\Sigma} \mathrm{d} s+\frac{\mu}{\sqrt{K}} \int_{\Sigma}\left(\boldsymbol{\beta} \overline{\boldsymbol{v}^{n+1}}\right) \cdot \bar{\varphi}_{\Sigma} \mathrm{d} s=\rho \int_{\Omega} \boldsymbol{f}^{n+1} \cdot \boldsymbol{\varphi} \mathrm{d} x
\end{aligned}
$$

for all $n \in \mathbb{N},(n+1) \delta t \leq T ; \quad \forall \boldsymbol{\varphi} \in \boldsymbol{W}$.

The Lax-Milgram theorem ensures the existence and uniqueness of a solution $\boldsymbol{v}^{n+1} \in \boldsymbol{W}$ satisfying (45). Moreover, by usual calculations, we get the two following stability estimates. First, taking $\varphi=2 \delta t \boldsymbol{v}^{n+1}$ in (45), we obtain using the Poincaré inequality in $\Omega_{f}$, for all $n \in \mathbb{N}$ such that $(n+1) \delta t \leq T$ :

$$
\begin{aligned}
\rho\left\|\boldsymbol{v}^{n+1}\right\|_{0, \Omega_{f}}^{2} & +\rho \sum_{k=0}^{n}\left\|\boldsymbol{v}^{k+1}-\boldsymbol{v}^{k}\right\|_{0, \Omega_{f}}^{2}+\mu \sum_{k=0}^{n} \delta t\left\|\nabla \boldsymbol{v}^{k+1}\right\|_{0, \Omega_{f}}^{2}+2 \mu_{m} \sum_{k=0}^{n} \delta t\left\|\nabla \boldsymbol{v}^{k+1}\right\|_{0, \Omega_{p}}^{2} \\
& +\mu k_{m} \sum_{k=0}^{n} \delta t\left\|\boldsymbol{v}^{k+1}\right\|_{0, \Omega_{p}}^{2}+\frac{2 \mu \alpha_{m}}{\sqrt{K}} \sum_{k=0}^{n} \delta t\left\|\llbracket \boldsymbol{v}^{k+1} \rrbracket_{\Sigma}\right\|_{0, \Sigma}^{2}+\frac{2 \mu \beta_{m}}{\sqrt{K}} \sum_{k=0}^{n} \delta t\left\|\overline{\boldsymbol{v}^{k+1}} \Sigma\right\|_{0, \Sigma}^{2} \\
& \leq \rho\left\|\boldsymbol{v}_{0}^{f}\right\|_{0, \Omega_{f}}^{2}+\frac{\rho^{2} c\left(\Omega_{f}\right)^{2}}{\mu} \sum_{k=0}^{n} \delta t\left\|\boldsymbol{f}^{k+1}\right\|_{0, \Omega_{f}}^{2}+\frac{\rho^{2}}{\mu k_{m}} \sum_{k=0}^{n} \delta t\left\|\boldsymbol{f}^{k+1}\right\|_{0, \Omega_{p}}^{2} \\
& \leq C_{0}\left(\Omega, T, \rho, \mu, k_{m}, \boldsymbol{f}, \boldsymbol{v}_{0}^{f}\right) .
\end{aligned}
$$

Second, for the sake of simplicity, we shall neglect the gravity force in $\Omega_{p}$ and thus consider the case with $\boldsymbol{f}^{p}=0$ in $\Omega_{p}$. Now taking $\boldsymbol{\varphi}=2\left(\boldsymbol{v}^{n+1}-\boldsymbol{v}^{n}\right)$ in (45), the right-hand side term can be estimated as below :

$$
2 \rho \int_{\Omega_{f}} \boldsymbol{f}^{k+1} \cdot\left(\boldsymbol{v}^{k+1}-\boldsymbol{v}^{k}\right) \mathrm{d} x \leq \rho \delta t\left\|\frac{\boldsymbol{v}^{k+1}-\boldsymbol{v}^{k}}{\delta t}\right\|_{0, \Omega_{f}}^{2}+\rho \delta t\left\|\boldsymbol{f}^{k+1}\right\|_{0, \Omega_{f}}^{2} .
$$


Then, we get for all $n \in \mathbb{N}$ such that $(n+1) \delta t \leq T$ :

$$
\begin{aligned}
\rho \sum_{k=0}^{n} \delta t\left\|\frac{\boldsymbol{v}^{k+1}-\boldsymbol{v}^{k}}{\delta t}\right\|_{0, \Omega_{f}}^{2} & +\mu\left\|\nabla \boldsymbol{v}^{n+1}\right\|_{0, \Omega_{f}}^{2}+\mu \sum_{k=0}^{n}\left\|\nabla\left(\boldsymbol{v}^{k+1}-\boldsymbol{v}^{k}\right)\right\|_{0, \Omega_{f}}^{2} \\
& +\mu_{m}\left\|\nabla \boldsymbol{v}^{n+1}\right\|_{0, \Omega_{p}}^{2}+\mu_{m} \sum_{k=0}^{n}\left\|\nabla\left(\boldsymbol{v}^{k+1}-\boldsymbol{v}^{k}\right)\right\|_{0, \Omega_{p}}^{2} \\
& +\mu k_{m}\left\|\boldsymbol{v}^{n+1}\right\|_{0, \Omega_{p}}^{2}+\mu k_{m} \sum_{k=0}^{n}\left\|\boldsymbol{v}^{k+1}-\boldsymbol{v}^{k}\right\|_{0, \Omega_{p}}^{2} \\
& +\frac{\mu \alpha_{m}}{\sqrt{K}}\left\|\llbracket \boldsymbol{v}^{n+1} \rrbracket_{\Sigma}\right\|_{0, \Sigma}^{2}+\frac{\mu \alpha_{m}}{\sqrt{K}} \sum_{k=0}^{n}\left\|\llbracket \boldsymbol{v}^{k+1}-\boldsymbol{v}^{k} \rrbracket_{\Sigma}\right\|_{0, \Sigma}^{2} \\
& +\frac{\mu \beta_{m}}{\sqrt{K}}\left\|\overline{\boldsymbol{v}^{n+1}}\right\|_{\Sigma, \Sigma}^{2}+\frac{\mu \beta_{m}}{\sqrt{K}} \sum_{k=0}^{n} \| \overline{\left(\boldsymbol{v}^{k+1}-\boldsymbol{v}^{k}\right)_{\Sigma} \|_{0, \Sigma}^{2}} \\
& \leq \mu\left\|\nabla \boldsymbol{v}_{0}\right\|_{0, \Omega_{f}}^{2}+\mu_{M}\left\|\nabla \boldsymbol{v}_{0}\right\|_{0, \Omega_{p}}^{2}+\mu k_{M}\left\|\boldsymbol{v}_{0}\right\|_{0, \Omega_{p}}^{2}+\frac{\mu \alpha_{M}}{\sqrt{K}}\left\|\llbracket \boldsymbol{v}_{0} \rrbracket_{\Sigma}\right\|_{0, \Sigma}^{2} \\
& +\frac{\mu \beta_{M}}{\sqrt{K}}\left\|\overline{\boldsymbol{v}_{0}}\right\|_{0, \Sigma}^{2}+\rho \sum_{k=0}^{n} \delta t\left\|\boldsymbol{f}^{k+1}\right\|_{0, \Omega_{f}}^{2} \\
& \leq C_{1}\left(\Omega, T, \rho, \mu, \mu_{M}, k_{M}, \alpha_{M}, \beta_{M}, \boldsymbol{f}, \boldsymbol{v}_{0}\right) .
\end{aligned}
$$

These bounds are sufficient to pass to the limit when $\delta t \rightarrow 0$ in such a semi-discrete linear scheme using only the weak compactness theorem and the weak limits, see e.g. [49, Chap. 4.1]. For any integer $N \in \mathbb{N}$, we set $\delta t=\delta t_{N}=T /(N+1)$ such that $\delta t \rightarrow 0^{+}$when $N \rightarrow+\infty$; further, we omit the index $N$ for sake of simplicity in the notations and just say $\delta t \rightarrow 0$. Let us consider the partition of the interval $[0, T]$ formed by the points $t_{k}=k \delta t$ for $k=0, \cdots, N+1$. By considering the solutions of the discrete scheme (45), let us construct the sequences of approximate solutions $\left(\boldsymbol{v}_{\delta t}\right)$ and $\left(\widetilde{\boldsymbol{v}}_{\delta t}\right)$ (for $\left.\delta t=\delta t_{N}, N=0,1,2, \cdots\right)$ which are functions from $[0, T]$ into $\boldsymbol{W}$ defined by:

$$
\begin{aligned}
& \left.\left.\boldsymbol{v}_{\delta t}(0):=\boldsymbol{v}^{0}=\boldsymbol{v}_{0}, \quad \boldsymbol{v}_{\delta t}(t):=\boldsymbol{v}^{k+1}, \quad \forall t \in\right] t_{k}, t_{k+1}\right], \quad k=0, \cdots, N \\
& \widetilde{\boldsymbol{v}}_{\delta t}(t):=\frac{t_{k+1}-t}{\delta t} \boldsymbol{v}^{k}+\frac{t-t_{k}}{\delta t} \boldsymbol{v}^{k+1}, \quad \forall t \in\left[t_{k}, t_{k+1}\right], \quad k=0, \cdots, N .
\end{aligned}
$$

Thus, $\boldsymbol{v}_{\delta t}$ is piecewise constant on $[0, T], \widetilde{\boldsymbol{v}}_{\delta t}$ is piecewise linear such that $\widetilde{\boldsymbol{v}}_{\delta t}\left(t_{k}\right)=\boldsymbol{v}^{k}$ for all $k=0, \cdots, N+1$, continuous in $[0, T]$, differentiable almost everywhere in $[0, T]$ and we have:

$$
\left.\frac{d \widetilde{\boldsymbol{v}}_{\delta t}}{d t}(t)=\frac{\boldsymbol{v}^{k+1}-\boldsymbol{v}^{k}}{\delta t}, \quad \forall t \in\right] t_{k}, t_{k+1}[, \quad k=0, \cdots, N
$$

We also define the sequence $\left(\boldsymbol{f}_{\delta t}\right)$ of functions in $L^{2}\left(0, T ; L^{2}(\Omega)^{d}\right)$ by: $\boldsymbol{f}_{\delta t}(t):=\boldsymbol{f}^{k+1}$ for all $\left.\left.t \in\right] t_{k}, t_{k+1}\right]$, $k=0, \cdots, N$, which are piecewise constant on $[0, T]$. Then, from the bounds (46) and (47) with the weak 
compactness theorem, we have the convergence results for these sequences as $\delta t=\delta t_{N} \rightarrow 0$ when $N \rightarrow+\infty$ :

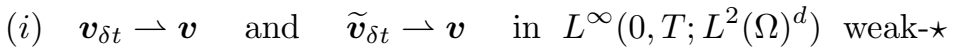

$$
\begin{aligned}
& \text { (ii) } \boldsymbol{v}_{\delta t} \rightarrow \boldsymbol{v} \text { and } \quad \widetilde{\boldsymbol{v}}_{\delta t} \rightarrow \boldsymbol{v} \quad \text { in } L^{2}(0, T ; \boldsymbol{W}) \text { weak } \\
& \text { (iii) } \frac{d \widetilde{\boldsymbol{v}}_{\delta t}^{f}}{d t} \rightarrow \frac{d \boldsymbol{v}^{f}}{d t} \quad \text { in } L^{2}\left(0, T ; L^{2}\left(\Omega_{f}\right)^{d}\right) \text { weak } \\
& \text { (iv) } \quad \boldsymbol{v}_{\delta t} \longrightarrow \boldsymbol{v} \text { and } \quad \widetilde{\boldsymbol{v}}_{\delta t} \longrightarrow \boldsymbol{v} \quad \text { in } L^{2}\left(0, T ; L^{2}(\Omega)^{d}\right) \text { strong } \\
& (v) \quad f_{\delta t} \longrightarrow \boldsymbol{f} \quad \text { in } L^{2}\left(0, T ; L^{2}(\Omega)^{d}\right) \text { strong. }
\end{aligned}
$$

Let us point out that $(i),(i i)$ and (iii) are obtained using (46) with the estimate below :

$$
\int_{0}^{T}\left\|\widetilde{\boldsymbol{v}}_{\delta t}(t)-\boldsymbol{v}_{\delta t}(t)\right\|_{0, \Omega_{f}}^{2} \mathrm{~d} t=\frac{\delta t}{3} \sum_{k=0}^{N}\left\|\boldsymbol{v}^{k+1}-\boldsymbol{v}^{k}\right\|_{0, \Omega_{f}}^{2} \leq \frac{C_{0} \delta t}{3 \rho} .
$$

This shows that $\left(\widetilde{\boldsymbol{v}}_{\delta t}-\boldsymbol{v}_{\delta t}\right) \longrightarrow 0$ in $L^{2}\left(0, T ; L^{2}\left(\Omega_{f}\right)^{d}\right)$ when $\delta t \rightarrow 0$ and thus $\left(\widetilde{\boldsymbol{v}}_{\delta t}\right)$ and $\left(\boldsymbol{v}_{\delta t}\right)$ have the same limit $\boldsymbol{v}$. The strong convergence in $(i v)$ holds from Aubin-Lions compactness theorem since the imbedding of $H^{1}(\Omega)^{d}$ into $L^{2}(\Omega)^{d}$ is compact. The point $(v)$ is classically proved by a density argument. The points $(i i)$, (iii) and $(v)$ are sufficient to take the limit of the discrete scheme (45) when $\delta t \rightarrow 0$.

That proves the existence of $\boldsymbol{v} \in L^{2}(0, T ; \boldsymbol{W})$ such that $\frac{d \boldsymbol{v}^{f}}{d t} \in L^{2}\left(0, T ; L^{2}\left(\Omega_{f}\right)^{d}\right)$ and $\boldsymbol{v}(t=0)=\boldsymbol{v}_{0} \in \boldsymbol{W}$ and verifying :

$$
\begin{aligned}
\rho \int_{\Omega_{f}} \frac{d \boldsymbol{v}}{d t} \cdot \boldsymbol{\varphi} \mathrm{d} x+\mu \int_{\Omega_{f}} \nabla \boldsymbol{v}: \nabla \boldsymbol{\varphi} \mathrm{d} x & +\int_{\Omega_{p}} \widetilde{\mu}^{p} \nabla \boldsymbol{v}: \nabla \boldsymbol{\varphi} \mathrm{d} x+\mu \int_{\Omega_{p}}\left(\boldsymbol{K}^{-1} \boldsymbol{v}\right) \cdot \boldsymbol{\varphi} \mathrm{d} x \\
& +\frac{\mu}{\sqrt{K}} \int_{\Sigma} \alpha \llbracket \boldsymbol{v} \rrbracket_{\Sigma} \cdot \llbracket \boldsymbol{\varphi} \rrbracket_{\Sigma} \mathrm{d} s+\frac{\mu}{\sqrt{K}} \int_{\Sigma}\left(\boldsymbol{\beta} \overline{\boldsymbol{v}}_{\Sigma}\right) \cdot \overline{\boldsymbol{\varphi}}_{\Sigma} \mathrm{d} s=\rho \int_{\Omega} \boldsymbol{f} \cdot \boldsymbol{\varphi} \mathrm{d} x,
\end{aligned}
$$

for a.e. $t \in] 0, T[; \quad \forall \boldsymbol{\varphi} \in \boldsymbol{W}$.

Then, it remains to pass to the limit when $\widetilde{\mu}^{p}:=\varepsilon \rightarrow 0$ in (48) to get the existence of velocity satisfying (41) with the $H^{2}$-regularity of pressure in $\Omega_{p}$. That follows the guidelines of the proof of Theorem 3.1.

\section{Stokes/Darcy Problem With Simplified interface CONDitions}

In this section, Saffman's approximation of (34) is considered assuming that $\left\|\boldsymbol{v}_{\Sigma}^{\star}(t) \cdot \boldsymbol{\tau}_{j}\right\|_{0, \Sigma} \ll\left\|\boldsymbol{v}_{\mid \Sigma}^{f}(t) \cdot \boldsymbol{\tau}_{j}\right\|_{0, \Sigma}$, for a.e. $t \in[0, T]$, and thus : $\llbracket \boldsymbol{v} \cdot \boldsymbol{\tau}_{j} \rrbracket_{\Sigma}^{\star} \approx \boldsymbol{v}_{\mid \Sigma}^{f} \cdot \boldsymbol{\tau}_{j}$, for $1 \leq j \leq d-1$, and $\overline{\boldsymbol{v}}_{\Sigma}^{\star} \approx \boldsymbol{v}_{\mid \Sigma}^{f} / 2$. Then, the problem reads:

$$
\left\{\begin{aligned}
\boldsymbol{\nabla} \cdot \boldsymbol{v} & =0 & & \text { in }(0, T) \times \Omega, \\
\rho \partial_{t} \boldsymbol{v}-\mu \Delta \boldsymbol{v}+\nabla p & =\rho \boldsymbol{f} & & \text { in }(0, T) \times \Omega_{f}, \\
\rho \partial_{t} \boldsymbol{v}+\mu \boldsymbol{K}^{-1} \boldsymbol{v}+\nabla p & =\rho \boldsymbol{f} & & \text { in }(0, T) \times \Omega_{p}, \\
\boldsymbol{v} & =0 & & \text { on }(0, T) \times \Gamma, \\
\boldsymbol{v}(t=0) & =\boldsymbol{v}_{0} \in \boldsymbol{W} & & \text { in } \Omega,
\end{aligned}\right.
$$




$$
\left\{\begin{aligned}
\llbracket \boldsymbol{v} \cdot \boldsymbol{n} \rrbracket_{\Sigma} & =0, \\
\llbracket \boldsymbol{\sigma}(\boldsymbol{v}, p) \cdot \boldsymbol{n} \rrbracket_{\Sigma}:=\left(\mu \nabla \boldsymbol{v}^{f} \cdot \boldsymbol{n}-p^{f} \boldsymbol{n}\right)_{\mid \Sigma}+p_{\mid \Sigma}^{p} \boldsymbol{n} & =\frac{\mu}{2 \sqrt{K}} \boldsymbol{\beta} \boldsymbol{v}_{\mid \Sigma}^{f}, \quad \text { on } \quad(0, T) \times \Sigma .
\end{aligned}\right.
$$

where :

$$
\boldsymbol{\sigma}(\boldsymbol{v}, p)^{f} \cdot \boldsymbol{n}_{\mid \Sigma}:=\left(\mu \nabla \boldsymbol{v}^{f} \cdot \boldsymbol{n}-p^{f} \boldsymbol{n}\right)_{\mid \Sigma}, \quad \text { and } \quad \boldsymbol{\sigma}(\boldsymbol{v}, p)^{p} \cdot \boldsymbol{n}_{\mid \Sigma}:=-p_{\mid \Sigma}^{p} \boldsymbol{n} .
$$

In this case, the tangential velocity jump is no longer taken into account explicitly in the simplified interface conditions (50) that only include the stress vector jump through $\Sigma$. Therefore, the general weak form (21) with test functions in $\boldsymbol{W}$ can be reduced to test functions $\varphi \in \boldsymbol{V} \hookrightarrow \boldsymbol{W}$ verifying by definition $\varphi_{\mid \Sigma}^{f}=\varphi_{\mid \Sigma}^{p}$. It yields, still looking for $\boldsymbol{v}(t) \in \boldsymbol{U}$ :

$$
\begin{aligned}
\rho\left\langle\partial_{t} \boldsymbol{v}, \boldsymbol{\varphi}\right\rangle_{\boldsymbol{U}^{\prime}, \boldsymbol{U}}+\mu \int_{\Omega_{f}} \nabla \boldsymbol{v}: \nabla \boldsymbol{\varphi} \mathrm{d} x & +\mu \int_{\Omega_{p}}\left(\boldsymbol{K}^{-1} \boldsymbol{v}\right) \cdot \boldsymbol{\varphi} \mathrm{d} x \\
& +\left\langle\llbracket \boldsymbol{\sigma}(\boldsymbol{v}, p) \cdot \boldsymbol{n} \rrbracket_{\Sigma}, \boldsymbol{\varphi}_{\mid \Sigma}^{f}\right\rangle_{-1 / 2, \Sigma}=\rho \int_{\Omega} \boldsymbol{f} \cdot \boldsymbol{\varphi} \mathrm{d} x, \quad \forall \boldsymbol{\varphi} \in \boldsymbol{V} .
\end{aligned}
$$

Let us consider the functional setting below still identifying the dual space $\boldsymbol{H}^{\prime}$ to $\boldsymbol{H}$ :

$$
\boldsymbol{V} \hookrightarrow \boldsymbol{W} \hookrightarrow \boldsymbol{U} \hookrightarrow \boldsymbol{H} \simeq \boldsymbol{H}^{\prime} \hookrightarrow \boldsymbol{U}^{\prime} \hookrightarrow \boldsymbol{W}^{\prime} \hookrightarrow \boldsymbol{V}^{\prime},
$$

and observe that Eq. (51) makes sense also for any $\varphi \in \boldsymbol{U}$. Then, it is more convenient for the analysis to extend (51) to the larger space $\boldsymbol{U}$ of test functions to have the same space as the solution space.

Now incorporating the interface conditions (50), we propose the following weak formulation for the Stokes/Darcy problem (49-50) with $\boldsymbol{f} \in L^{2}\left(0, T ; L^{2}(\Omega)^{d}\right)$ using divergence-free test functions in $\boldsymbol{U}$.

Find $\boldsymbol{v} \in \mathcal{C}([0, T] ; \boldsymbol{H}) \cap L^{2}(0, T ; \boldsymbol{U})$ with $\boldsymbol{v}^{\prime}:=\frac{d \boldsymbol{v}}{d t} \in L^{2}\left(0, T ; \boldsymbol{U}^{\prime}\right)$ such that $\boldsymbol{v}(t=0)=\boldsymbol{v}_{0}$ in $\boldsymbol{H}$, and that satisfy for all $\boldsymbol{\varphi} \in \boldsymbol{U}$ :

$$
\begin{aligned}
& \rho\left\langle\frac{d \boldsymbol{v}}{d t}, \boldsymbol{\varphi}\right\rangle_{\boldsymbol{U}^{\prime}, \boldsymbol{U}}+\mu \int_{\Omega_{f}} \nabla \boldsymbol{v}: \nabla \boldsymbol{\varphi} \mathrm{d} x+\mu \int_{\Omega_{p}}\left(\boldsymbol{K}^{-1} \boldsymbol{v}\right) \cdot \boldsymbol{\varphi} \mathrm{d} x+\frac{\mu}{2 \sqrt{K}} \int_{\Sigma}\left(\boldsymbol{\beta} \boldsymbol{v}_{\mid \Sigma}^{f}\right) \cdot \boldsymbol{\varphi}_{\mid \Sigma}^{f} \mathrm{~d} s \\
&\left.=\rho \int_{\Omega} \boldsymbol{f} \cdot \boldsymbol{\varphi} \mathrm{d} x, \quad \text { for a.e. } t \in\right] 0, T[; \boldsymbol{\varphi} \in \boldsymbol{U} .
\end{aligned}
$$

Then, we have the following solvability result.

Theorem 4.1 (Well-posedness of the Stokes/Darcy problem with simplified interface conditions). For any $\boldsymbol{v}_{0} \in \boldsymbol{U}$ in addition to the basic assumptions on the data $\boldsymbol{f}, \boldsymbol{K}, \boldsymbol{\beta}$, there exists a unique solution $(\boldsymbol{v}, p)$ to the problem (49-50) such that $\boldsymbol{v} \in \mathcal{C}([0, T] ; \boldsymbol{H}) \cap L^{\infty}(0, T ; \boldsymbol{U})$ with $\boldsymbol{v}^{\prime} \in L^{2}(0, T ; \boldsymbol{H})$ and $p \in L^{2}\left(0, T ; L^{2}(\Omega)\right)$ with $p^{p} \in L^{2}\left(0, T ; H^{1}\left(\Omega_{p}\right)\right)$, which satisfies the weak form (52) and the energy equality below :

$$
\begin{array}{r}
\frac{\rho}{2}\|\boldsymbol{v}(t)\|_{0, \Omega}^{2}+\mu \int_{0}^{t} \int_{\Omega_{f}}|\nabla \boldsymbol{v}|^{2} \mathrm{~d} x \mathrm{~d} \tau+\mu \int_{0}^{t} \int_{\Omega_{p}}\left(\boldsymbol{K}^{-1} \boldsymbol{v}\right) \cdot \boldsymbol{v} \mathrm{d} x \mathrm{~d} \tau+\frac{\mu}{2 \sqrt{K}} \int_{0}^{t} \int_{\Sigma}\left(\boldsymbol{\beta} \boldsymbol{v}_{\mid \Sigma}^{f}\right) \cdot \boldsymbol{v}_{\mid \Sigma}^{f} \mathrm{~d} s \mathrm{~d} \tau \\
=\frac{\rho}{2}\left\|\boldsymbol{v}_{0}\right\|_{0, \Omega}^{2}+\rho \int_{0}^{t} \int_{\Omega} \boldsymbol{f} \cdot \boldsymbol{v} \mathrm{d} x \mathrm{~d} \tau, \quad \text { for all } t \in[0, T] .
\end{array}
$$

Moreover, there exists a function $q \in L^{2}\left(0, T ; L^{2}(\Omega)\right)$ with each restriction $q^{f} \in L^{2}\left(0, T ; L_{0}^{2}\left(\Omega_{f}\right)\right)$ and $q^{p} \in$ $L^{2}\left(0, T ; L_{0}^{2}\left(\Omega_{p}\right) \cap H^{1}\left(\Omega_{p}\right)\right)$ both defined uniquely up to an additive constant, such that the pressure field $p \in$ $L^{2}\left(0, T ; L^{2}(\Omega)\right)$ with $p^{p} \in L^{2}\left(0, T ; H^{1}\left(\Omega_{p}\right)\right)$ is defined uniquely by :

$$
p_{\mid \Omega_{f}}:=q^{f}+\frac{\Pi_{0}}{2}, \quad p_{\mid \Omega_{p}}:=q^{p}-\frac{\Pi_{0}}{2}, \quad \text { such that } \llbracket p-q \rrbracket_{\Sigma}=\Pi_{0},
$$


with $\boldsymbol{\sigma}(\boldsymbol{v}, p)^{f} \cdot \boldsymbol{n}_{\mid \Sigma} \in L^{2}\left(0, T ; L^{2}(\Sigma)^{d}\right)$, where the constant quantity $\Pi_{0}$ is defined from $(\boldsymbol{v}, q)$ by :

$$
\Pi_{0}:=\frac{1}{|\Sigma|}\left\langle\llbracket \boldsymbol{\sigma}(\boldsymbol{v}, q) \cdot \boldsymbol{n} \rrbracket_{\Sigma}-\frac{\mu}{2 \sqrt{K}} \boldsymbol{\beta} \boldsymbol{v}_{\mid \Sigma}^{f}, \boldsymbol{n}\right\rangle_{-1 / 2, \Sigma} .
$$

Proof. It is similar to the proof made in details for Theorem 2.5, and we shall sketch the different steps by adapting them to the present case.

- First, Lions' Theorem 2.3 ensures the existence and uniqueness of the solution $\boldsymbol{v}$ to the weak problem (52). Indeed, the required continuity and coercivity over $\boldsymbol{U}$ of the bilinear form $a(.,$.$) corresponding to$ the elliptic operator in (52) are obtained with the basic assumptions on the data through standard calculations using Cauchy-Schwarz inequality, Poincaré inequality in $H_{0 \Gamma}^{1}\left(\Omega_{f}\right)^{d}$, and classical trace inequality on $\Sigma$ for functions in $H^{1}\left(\Omega_{f}\right)^{d}$.

- With $\boldsymbol{f} \in L^{2}\left(0, T ; L^{2}(\Omega)^{d}\right)$ and $\boldsymbol{v}_{0} \in \boldsymbol{U}$, choosing in (52) the test function $\boldsymbol{\varphi}=2 \boldsymbol{v}^{\prime}(t) \in \boldsymbol{U}$, we get by standard calculations similar as in the proof of Theorem 2.5 :

$$
\begin{array}{r}
\rho \int_{0}^{t}\left\|\frac{d \boldsymbol{v}}{d t}(\tau)\right\|_{0, \Omega}^{2} \mathrm{~d} \tau \\
+\mu \int_{\Omega_{f}}|\nabla \boldsymbol{v}(t)|^{2} \mathrm{~d} x+\mu \int_{\Omega_{p}}\left(\boldsymbol{K}^{-1} \boldsymbol{v}(t)\right) \cdot \boldsymbol{v}(t) \mathrm{d} x+\frac{\mu}{2 \sqrt{K}} \int_{\Sigma}\left(\boldsymbol{\beta} \boldsymbol{v}_{\mid \Sigma}^{f}(t)\right) \cdot \boldsymbol{v}_{\mid \Sigma}^{f}(t) \mathrm{d} s \\
\leq \mu\left\|\nabla \boldsymbol{v}_{0}\right\|_{0, \Omega_{f}}^{2}+\mu k_{M}\left\|\boldsymbol{v}_{0}\right\|_{0, \Omega_{p}}^{2}+\frac{\mu \beta_{M}}{2 \sqrt{K}}\left\|\boldsymbol{v}_{0 \mid \Sigma}^{f}\right\|_{0, \Sigma}^{2}+\rho \int_{0}^{t}\|\boldsymbol{f}(\tau)\|_{0, \Omega}^{2} \mathrm{~d} \tau \\
\left.\leq C\left(\Omega, \Omega_{f}, \Omega_{p}, T, \rho, \mu, \boldsymbol{K}, \boldsymbol{\beta}, \boldsymbol{v}_{0}, \boldsymbol{f}\right), \quad \text { for a.e. } t \in\right] 0, T[
\end{array}
$$

This shows that $\boldsymbol{v}^{\prime} \in L^{2}(0, T ; \boldsymbol{H})$ and $\nabla \boldsymbol{v}^{f} \in L^{\infty}\left(0, T ; L^{2}\left(\Omega_{f}\right)^{d}\right)$. Hence, using Poincaré inequality in $\Omega_{f}$, we have also $\boldsymbol{v} \in L^{\infty}(0, T ; \boldsymbol{U})$.

- Then, the energy equality (53) is easily obtained by taking the test function $\boldsymbol{\varphi}=\boldsymbol{v}(t) \in \boldsymbol{U}$ in (52) and integrating in time over $(0, t)$ for all $t \in[0, T]$ with the initial condition.

- To interpret the weak problem (52), let us take any $\varphi \in \boldsymbol{U}$ in (52) such that $\varphi^{f} \in H_{0}^{1}\left(\Omega_{f}\right)^{d}$ and $\varphi^{p}=0$. So, we have for a.e. $t \in] 0, T[$ :

$$
\rho \int_{\Omega_{f}} \frac{d \boldsymbol{v}}{d t} \cdot \boldsymbol{\varphi} \mathrm{d} x+\mu \int_{\Omega_{f}} \nabla \boldsymbol{v}: \nabla \boldsymbol{\varphi} \mathrm{d} x=\rho \int_{\Omega_{f}} \boldsymbol{f} \cdot \boldsymbol{\varphi} \mathrm{d} x
$$

which gives by integration by parts :

$$
\rho \int_{\Omega_{f}} \frac{d \boldsymbol{v}}{d t} \cdot \boldsymbol{\varphi} \mathrm{d} x-\mu \int_{\Omega_{f}} \Delta \boldsymbol{v} \cdot \boldsymbol{\varphi} \mathrm{d} x=\rho \int_{\Omega_{f}} \boldsymbol{f} \cdot \boldsymbol{\varphi} \mathrm{d} x .
$$

Applying De Rham's theorem in $\Omega_{f}$ since $\boldsymbol{\nabla} \cdot \boldsymbol{\varphi}=0$, there exists $q^{f}(t) \in L_{0}^{2}\left(\Omega_{f}\right)$ which is unique since the domain $\Omega_{f}$ is connected, such that the equation below holds at least in the sense of $H^{-1}\left(\Omega_{f}\right)^{d}$ for a.e. $t \in] 0, T[$ :

$$
\rho \frac{\partial \boldsymbol{v}^{f}}{\partial t}-\mu \Delta \boldsymbol{v}^{f}+\nabla q^{f}=\rho \boldsymbol{f}, \quad \text { in }(0, T) \times \Omega_{f} .
$$

Thus, the Stokes equation is satisfied in $(0, T) \times \Omega_{f}$.

Doing similarly, we take any $\varphi \in \boldsymbol{U}$ in (52) such that $\varphi^{p} \in H_{0}^{1}\left(\Omega_{p}\right)^{d}$ and $\varphi^{f}=0$, and apply De Rham's theorem in $\Omega_{p}$. Then, there exists $q^{p}(t) \in L_{0}^{2}\left(\Omega_{p}\right)$ which is unique since the domain $\Omega_{p}$ is connected, such that the Darcy below holds at least in the sense of $H^{-1}\left(\Omega_{p}\right)^{d}$ for a.e. $\left.t \in\right] 0, T[$ :

$$
\rho \frac{\partial \boldsymbol{v}^{p}}{\partial t}+\mu \boldsymbol{K}^{-1} \boldsymbol{v}^{p}+\nabla q^{p}=\rho \boldsymbol{f}, \quad \text { in }(0, T) \times \Omega_{p} .
$$


Moreover, from the regularity of the other terms in this equation which all belong to $L^{2}\left(0, T ; L^{2}\left(\Omega_{p}\right)^{d}\right)$, it yields also that $\nabla q^{p} \in L^{2}\left(0, T ; L^{2}\left(\Omega_{p}\right)^{d}\right)$ and thus $q^{p} \in L^{2}\left(0, T ; H^{1}\left(\Omega_{p}\right)\right)$.

This defines the pressure field $q \in L^{2}\left(0, T ; L^{2}(\Omega)\right)$ by its restrictions $q^{f} \in L^{2}\left(0, T ; L_{0}^{2}\left(\Omega_{f}\right)\right)$ over $\Omega_{f}$ and $q^{p} \in L^{2}\left(0, T ; L_{0}^{2}\left(\Omega_{p}\right) \cap H^{1}\left(\Omega_{p}\right)\right)$ over $\Omega_{p}$, each defined uniquely up to an additive constant, and shows that $(\boldsymbol{v}, q)$ satisfies Eq. (49).

- It remains to recover the last interface condition in Eq. (50). Let us take any $\boldsymbol{\varphi} \in \boldsymbol{V} \subset \boldsymbol{U}$ in (52) and make the difference with Eq. (51) written with the pressure field $q$ to take account of the recovered Stokes/Darcy equations (49) satisfied with $(\boldsymbol{v}, q)$. We get :

$$
\left\langle\llbracket \boldsymbol{\sigma}(\boldsymbol{v}, q) \cdot \boldsymbol{n} \rrbracket_{\Sigma}, \boldsymbol{\varphi}_{\mid \Sigma}^{f}\right\rangle_{-1 / 2, \Sigma}-\frac{\mu}{2 \sqrt{K}} \int_{\Sigma}\left(\boldsymbol{\beta} \boldsymbol{v}_{\mid \Sigma}^{f}\right) \cdot \boldsymbol{\varphi}_{\mid \Sigma}^{f} \mathrm{~d} s=0, \quad \text { for all } \boldsymbol{\varphi} \in \boldsymbol{V} .
$$

Since the surface $\Sigma$ is closed, this is equivalent to

$$
\left\langle\llbracket \boldsymbol{\sigma}(\boldsymbol{v}, q) \cdot \boldsymbol{n} \rrbracket_{\Sigma}-\frac{\mu}{2 \sqrt{K}} \boldsymbol{\beta} \boldsymbol{v}_{\mid \Sigma}^{f}, \boldsymbol{\varphi}_{\mid \Sigma}\right\rangle_{-1 / 2, \Sigma}=0, \quad \text { for all } \boldsymbol{\varphi} \in H^{1}\left(\Omega_{p}\right)^{d} ; \quad \boldsymbol{\nabla} \cdot \boldsymbol{\varphi}=0 \text { in } \Omega_{p} .
$$

Applying now Lemma 2.4 in $\Omega_{p}$, for any $\boldsymbol{\psi} \in H^{1 / 2}(\Sigma)^{d}$, there exists $\varphi \in H^{1}\left(\Omega_{p}\right)^{d}$ with $\boldsymbol{\nabla} \cdot \boldsymbol{\varphi}=0$ in $\Omega_{p}$ such that

$$
\boldsymbol{\psi}=\boldsymbol{\varphi}_{\mid \Sigma}+\frac{1}{|\Sigma|}\left(\int_{\Sigma} \boldsymbol{\psi} \cdot \boldsymbol{n} \mathrm{d} s\right) \boldsymbol{n}
$$

Then, using this equality in the previous equation, we get :

$$
\left\langle\llbracket \boldsymbol{\sigma}(\boldsymbol{v}, q) \cdot \boldsymbol{n} \rrbracket_{\Sigma}-\frac{\mu}{2 \sqrt{K}} \boldsymbol{\beta} \boldsymbol{v}_{\mid \Sigma}^{f}, \boldsymbol{\psi}\right\rangle_{-1 / 2, \Sigma}-\frac{1}{|\Sigma|}\left\langle\llbracket \boldsymbol{\sigma}(\boldsymbol{v}, q) \cdot \boldsymbol{n} \rrbracket_{\Sigma}-\frac{\mu}{2 \sqrt{K}} \boldsymbol{\beta} \boldsymbol{v}_{\mid \Sigma}^{f}, \boldsymbol{n}\right\rangle_{-1 / 2, \Sigma} \int_{\Sigma} \boldsymbol{\psi} \cdot \boldsymbol{n} \mathrm{d} s=0
$$

By introducing the constant $\Pi_{0}$ as in Eq. (55), it yields :

$$
\left\langle\llbracket \boldsymbol{\sigma}(\boldsymbol{v}, q) \cdot \boldsymbol{n} \rrbracket_{\Sigma}-\frac{\mu}{2 \sqrt{K}} \boldsymbol{\beta} \boldsymbol{v}_{\mid \Sigma}^{f}-\Pi_{0} \boldsymbol{n}, \boldsymbol{\psi}\right\rangle_{-1 / 2, \Sigma}=0
$$

Then, defining the pressure field $p$ by Eq. (54) such that $\llbracket p-q \rrbracket_{\Sigma}=\Pi_{0}$, we get finally :

$$
\left\langle\llbracket \boldsymbol{\sigma}(\boldsymbol{v}, p) \cdot \boldsymbol{n} \rrbracket_{\Sigma}-\frac{\mu}{2 \sqrt{K}} \boldsymbol{\beta} \boldsymbol{v}_{\mid \Sigma}^{f}, \boldsymbol{\psi}\right\rangle_{-1 / 2, \Sigma}=0, \quad \text { for all } \boldsymbol{\psi} \in H^{1 / 2}(\Sigma)^{d}
$$

Hence, the stress vector jump condition in Eq. (50) is verified by the solution $(\boldsymbol{v}, p)$ and therefore, $\boldsymbol{\sigma}(\boldsymbol{v}, p)^{f} \cdot \boldsymbol{n}_{\mid \Sigma} \in L^{2}\left(0, T ; L^{2}(\Sigma)^{d}\right)$, which ends the proof.

\section{Conclusion}

In this work, we have revisited the mathematical analysis of the coupled unsteady and incompressible viscous fluid flow through the interface over a permeable medium. The study considers new jump interface conditions recently derived and shown to be physically meaningfull for the two- or three-dimensional flow in [11]. These conditions generalize Beavers \& Joseph and Ochoa-Tapia \& Whitaker conditions and include jumps of both stress and tangential velocity vectors at the interface. Hence, they are different from those most commonly used in the literature.

Two interface models are proved to be globally well-posed in time depending upon the flow in the porous medium should be described by Brinkman's law or Darcy's law and coupled to the Stokes flow of a free fluid. 
Moreover, a simplified Stokes/Darcy interface model using classical Saffman's approximation, but also different from the usual one, is analysed due to its practical interest in many situations.

The extension of this work to take account of the inertial effects both in the pure fluid and porous regions is currently in progress, which is a very challenging open problem.

\section{APPENDIX A. BRINKMAN'S VISCOUS DIFFUSION TERM VERSUS DARCY'S DRAG}

Let us detail some arguments to support the discussion in Remark 1.1.

Among the numerous correlations of absolute permeability $K(\phi)$ versus porosity $\phi$, the well-known CarmanKozeny formula [28], confirmed in [51], for homogeneous and isotropic granular media composed of random packed beds of spherical solid particles reads, $d_{p}$ being the mean diameter of particles :

$$
\left\{\begin{array}{l}
\text { Carman-Kozeny's correlation for granular media : } \\
K(\phi)=\frac{d_{p}^{2} \phi^{3}}{180(1-\phi)^{2}} .
\end{array}\right.
$$

For fibrous materials, it is more suitable to use the following Happel-Langmuir's formula [41], $d_{p}$ being here the mean diameter of cylindrical fibres, or others discussed in [42] for arrays of cylinder rods aligned or normal to the flow :

$$
\left\{\begin{array}{c}
\text { Happel-Langmuir's correlation for fibrous media : } \\
K(\phi)=\frac{d_{p}^{2}}{16(1-\phi)}\left(-\ln (1-\phi)-\frac{3}{2}+2(1-\phi)-\frac{(1-\phi)^{2}}{2}\right) .
\end{array}\right.
$$

Then, the Darcy number Da depends on the macroscopic length scale $L$ at which the momentum transport is to be studied :

$$
\operatorname{Da}(\phi):=\frac{K(\phi)}{L^{2}}=\frac{K(\phi)}{d_{p}^{2}}\left(\frac{d_{p}}{L}\right)^{2}
$$

It is useful to plot the normalized permeability $K(\phi) / d_{p}^{2}$ as shown in Fig. 2.

The two correlations $K(\phi)$ have similar variations with porosity, both satisfy the required coherency at the asymptotics when $\phi \rightarrow 0$ or $\phi \rightarrow 1$, and as expected, the permeability of fibrous materials is larger than the one of granular media at the same porosity. As soon as we have $L=\mathcal{O}\left(100 d_{p}\right)$ or more, then we observe that $\mathrm{Da}(\phi) \ll 1$, except when $\phi \simeq 1$. In practice for many real configurations, we have $L=\mathcal{O}\left(1000 d_{p}\right)$ or more.

It is now useful to plot the ratio $R_{B / D}(\phi)$ in Eq. (11) to supply a validity criterium whether Brinkman's viscous term can be neglected with respect to Darcy's drag. Then, it appears in Fig. 3 that this approximation is fully justified except when $\phi \simeq 1$. That is the reason why all the terms have been kept in the transition layer for the derivation of the asymptotic interface model in [11].

\section{REFERENCES}

[1] V. Adolfsson And D. Jerison, $L^{p}$-Integrability of the second order derivatives for the Neumann problem in convex domains, Indiana Univ. Mathematics J. 43(4), 1123-1138 (1994).

[2] G. Allaire, Homogenization of the Navier-Stokes equations in open sets perforated with tiny holes. I: Abstract framework, a volume distribution of holes, Arch. Ration. Mech. Anal. 113(3), 209-259 (1991).

[3] G. Allaire, Homogenization of the Navier-Stokes equations in open sets perforated with tiny holes. II: Non-critical sizes of the holes for a volume distribution and a surface distribution of holes, Arch. Ration. Mech. Anal. 113(3), 261-298 (1991).

[4] Рh. Angot, Analysis of singular perturbations on the Brinkman problem for fictitious domain models of viscous flows, Math. Meth. in the Appl. Sci. 22(16), 1395-1412 (1999).

[5] Ph. Angot, A model of fracture for elliptic problems with flux and solution jumps, C. R. Acad. Sci. Paris, Ser. I Math. 337(6), 425-430 (2003).

[6] Рн. Angot, A unified fictitious domain model for general embedded boundary conditions, C. R. Acad. Sci. Paris, Ser. I Math. 341(11), 683-688 (2005). 


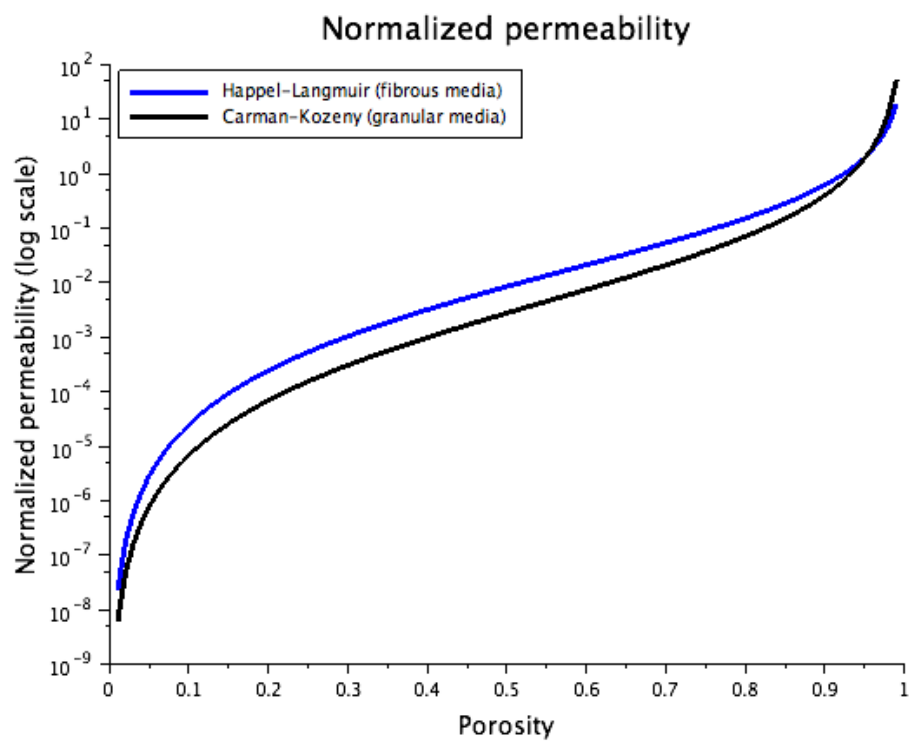

FiguRe 2. Normalized permeability $K(\phi) / d_{p}^{2}$ versus porosity $\phi$ with Carman-Kozeny's Eq. (57) (below), or Happel-Langmuir's Eq. (58) (above).

\section{Ratio of Brinkman viscous term over Darcy drag}

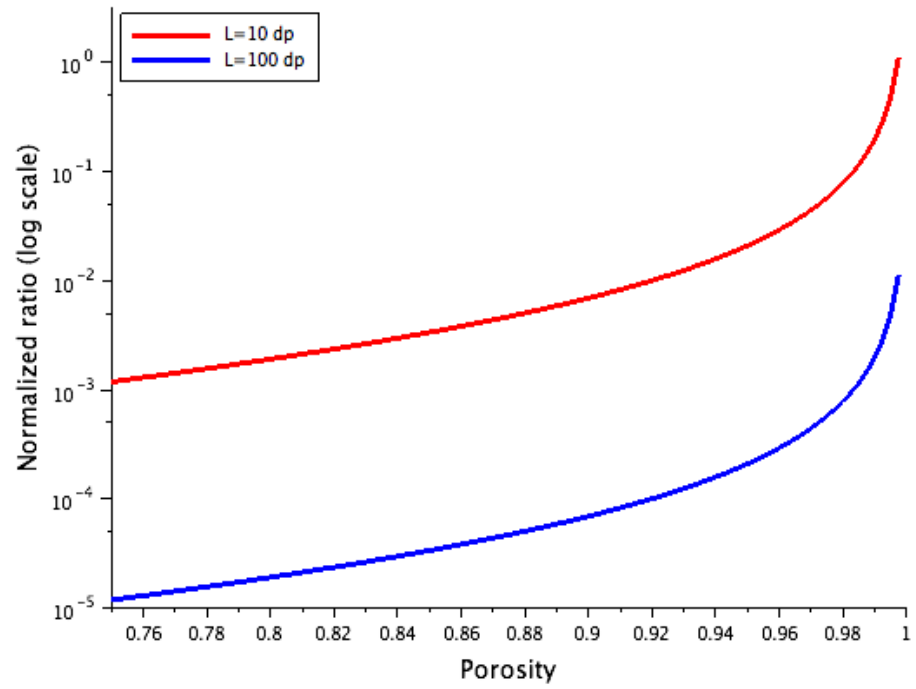

Figure 3. Ratio $R_{B / D}(\phi)$ versus porosity $\phi$ using Happel-Langmuir's Eq. (58) for different macroscopic scales : $L=10 d_{p}$ (above) and $L=100 d_{p}$ (below).

[7] Ph. Angot, A fictitious domain model for the Stokes/Brinkman problem with jump embedded boundary conditions, C. R. Math. Acad. Sci. Paris, Ser. I 348(11-12), 697-702 (2010).

[8] Рн. Angot, On the well-posed coupling between free fluid and porous viscous flows, Appl. Math. Lett. 24(6), 803-810 (2011). 
[9] Ph. Angot, F. Boyer and F. Hubert, Asymptotic and numerical modelling of flows in fractured porous media, Math. Model. Numer. Anal. 43(2), 239-275 (2009).

[10] Ph. Angot, G. Carbou and V. PÉron, Asymptotic study for Stokes-Brinkman model with jump embedded transmission conditions, Asymptotic Analysis 96(3-4), 223-249 (2016).

[11] Ph. Angot, B. Goyeau and A. J. Ochoa-Tapia, Asymptotic modeling of transport phenomena at the interface between a fluid and a porous layer: Jump conditions, Phys. Rev. E 95(6), 063302-1-16 (2017).

[12] T. Arbogast and D. S. Brunson, A computational method for approximating a Stokes-Darcy system governing a vuggy porous medium, Comput. Geosci. 11, 207-218 (2007).

[13] J.-L. Auriault, About the Beavers and Joseph boundary condition, Transp. in Porous Media 83, 257-266 (2010).

[14] L. Badea, M. Discacciati and A. Quarteroni, Mathematical analysis of the Navier-Stokes/Darcy coupling, Numer. Math. 1152, 195-227 (2010).

[15] J. Barrère, O. Gipouloux and S. Whitaker, On the closure problem for Darcy's law, Transp. Porous Media 7, $209-222$ (1992).

[16] G. S. Beavers and D. D. Joseph, Boundary conditions at a naturally permeable wall, J. Fluid Mech. 30, 197-207 (1967).

[17] A. Yu. Beliaev and S. M. Kozlov, Darcy equation for random porous media, Comm. Pure Appl. Math. XLIX, 1-34 (1996).

[18] C. Bernardi, T.-C. Rebollo, F. Hecht and Z. Mghazli, Mortar finite element discretization of a model coupling Darcy and Stokes equations, Math. Model. Numer. Anal. 42, 375-410 (2008).

[19] F. Boyer And P. FABrie, Mathematical Tools for the Study of the Incompressible Navier-Stokes Equations and Related Models, Appl. Math. Sci., 183, Springer, New York (2013).

[20] H. Brezis, Functional Analysis, Sobolev Spaces and Partial Differential Equations, Springer-Verlag, New York (2011).

[21] A. Brillard, Asymptotic analysis of incompressible and viscous fluid flow through porous media; Brinkman's law via epiconvergence methods, Annales Faculté des Sciences de Toulouse 8(2), 225-252 (1986).

[22] A. Brillard, J. El. Amrani and M. Jarroudi, Derivation of a contact law between a free fluid and thin porous layers via asymptotic analysis methods, Applicable Analysis 92(4), 665-689 (2013).

[23] H. C. Brinkman, A calculation of the viscous force exerted by a flowing fluid on a dense swarm of particules, Appl. Sci. Res. A1, 27-34 (1947).

[24] H. C. Brinkman, On the permeability of media consisting of closely packed porous particules, Appl. Sci. Res. A1, 81-86 (1947).

[25] J.-P. Caltagirone, Physique des Ecoulements Continus, Mathématiques et Applications 74, SMAI \& Springer-Verlag, Berlin (2013).

[26] Y. Cao, M. Gunzburger, F. Hua and X. Wang, Coupled Stokes-Darcy model with Beavers-Joseph interface boundary condition, Comm. Math. Sci. 8(1), 1-25 (2010).

[27] Y. Cao, M. Gunzburger, X. Hu, F. Hua, X. Wang and W. Zhao, Finite element approximations for Stokes-Darcy flow with Beavers-Joseph interface conditions, SIAM J. Numer. Anal. 47(6), 4239-4256 (2010).

[28] P. C. Carman, Fluid flow through granular beds, Trans. Inst. Chem. Eng. 15, 150-166 (1937).

[29] T. Carraro, C. Goll, A. Marciniak-Czochra and A. Mikelić, Pressure jump interface law for the Stokes-Darcy coupling: confirmation by direct numerical simulations, J. Fluid. Mech. 732, 510-536 (2013).

[30] M. Cieszko and J. Kubik, Derivation of matching conditions at the contact surface between fluid-saturated porous solid and bulk fluid, Transp. Porous Media 34, 319-336 (1999).

[31] R. Dautray and J.-L. Lions, Mathematical Analysis and Numerical Methods for Science and Technology. II Evolution problems, vol. 6, Springer-Verlag, Berlin (1993).

[32] M. Discacciati and A. Quarteroni, Navier-Stokes/Darcy coupling: modeling, analysis and numerical approximation, Rev. Math. Complut. 22(2), 315-426 (2009).

[33] M. Discacciati, A. Quarteroni and A. Valli, Robin-Robin domain decomposition methods for the Stokes-Darcy coupling, SIAM J. Numer. Anal. 45, 1246-1268 (2007).

[34] E. Fabes, O. Mendez and M. Mitrea, Boundary Layers on Sobolev-Besov Spaces and Poisson's Equation for the Laplacian in Lipschitz Domains, J. Functional Anal. 159(2), 323-368 (1998).

[35] V. Girault and P.A. Raviart, Finite Element Methods for the Navier-Stokes Equations, Springer Series in Comput. Math. 5, Springer-Verlag, Berlin (1986).

[36] V. Girault and B. Rivière, DG approximation of coupled Navier-Stokes and Darcy equations by Beavers-Joseph-Saffman interface condition, SIAM J. Numer. Anal. 47(3), 2052-2089 (2009).

[37] V. Girault, D. Vassilev and I. Yotov, Mortar multiscale finite element methods for Stokes-Darcy flows, Numer. Math. 127, 93-165 (2014).

[38] B. Goyeau, D. Lhulllier, D. Gobin and M. G. Velarde, Momentum transport at a fluid-porous interface, Int. J. Heat Mass Transfer 46, 4071-4081 (2003).

[39] W. G. Gray and K. O'Neill, On the general equations for flow in porous media and their reduction to Darcy's law, Water Resources Res. 12(2), 148-154 (1976).

[40] P. Grisvard, Elliptic problems in nonsmooth domains, Monographs and Studies in Mathematics 24, Adv. Publish. Prog., Pitman, Boston (1985). 
[41] J. HAPpel, Viscous flow relative to arrays of cylinders, AIChE J. 5, 174-177 (1959).

[42] G. W. JaCKSON AND D. F. James, The permeability of fibrous porous media, Can. J. Chem. Eng. 64, 364-374 (1986).

[43] W. JäGer And A. Mikelić, On the interface boundary condition of Beavers \& Joseph and Saffman, SIAM J. Appl. Math. 60(4), 1111-1127 (2000).

[44] W. JÄGER AND A. Mikelić, On the boundary conditions at the interface between a porous medium and a free fluid, Transp. Porous Media 78, 489-508 (2009).

[45] J. KubiK And M. CieszKo, Analysis of matching conditions at the boundary surface of a fluid-saturated porous solid and a fluid fluid: the use of Lagrange multipliers, Continuum Mech. Thermodyn. 17(4), 351-359 (2005).

[46] W. L. Layton, F. Schieweck and I. Yotov, Coupling fluid flow with porous media flow, SIAM J. Numer. Anal. 40(6), 2195-2218 (2003)

[47] T. LÉvy, Fluid flow through an array of fixed particules, Int. J. Engng Sci. 21, 11-23 (1983).

[48] T. Lévy and E. Sanchez-Palencia, Suspension of solid particules in a newtonian fluid, J. Non-Newtonian Fluid Mech. 13(1), 63-78 (1983).

[49] J.-L. Lions, Quelques méthodes de résolution des problèmes aux limites non linéaires, Dunod \& Gauthier-Villars, Paris (1969).

[50] J.-L. Lions And E. Magenes, Non-homogeneous boundary value problems and applications, vol. I, Springer-Verlag, New York (1972).

[51] M. J. MacDonald, C. C. Chu, P. P. Guilloit and K. M. NG, A generalized Blake-Kozeny equation for multi-sized spherical particles, AIChE J. 37, 1583-1588 (1991).

[52] A. Marciniak-Czochra and A. Mikelić, Effective pressure interface law for transport phenomena between an unconfined fluid and a porous medium using homogenization, SIAM Multiscale Model. Simul. 10, 285-305 (2012).

[53] J. NEČAS, Sur une méthode pour résoudre les équations aux dérivées partielles de type elliptique, voisine de la variationnelle, Ann. Scuola Norm. Sup. Pisa 16, 305-326 (1962).

[54] J. NeČAs, Les méthodes directes en théorie des équations elliptiques, Masson, Paris (1967).

[55] D. A. NIELD, The Beavers-Joseph boundary condition and related matters: a historical and critical review, Transp. in Porous Media 78, 537-540 (2009).

[56] J. A. OChoA-Tapia And S. Whitaker, Momentum transfer at the boundary between a porous medium and a homogeneous fluid I: theoretical development, Int. J. Heat Mass Transfer 38, 2635-2646 (1995).

[57] J. A. OchoA-Tapia And S. Whitaker, Momentum transfer at the boundary between a porous medium and a homogeneous fluid II: comparison with experiment, Int. J. Heat Mass Transfer 38, 2647-2655 (1995).

[58] L. E. Payne and B. Straughan, Analysis of the boundary condition at the interface between a viscous fluid and a porous medium and related modelling questions, J. Math. Pures Appl. 77, 317-354 (1998).

[59] B. Rivière And I. Yotov, Locally conservative coupling of Stokes and Darcy flow, SIAM J. Numer. Anal. 42, 1959-1977 (2005).

[60] J. Rubinstein, Effective equations for flow in random porous media with large number of scales, J. Fluid Mech. 170, 379-383 (1986).

[61] J. Rubinstein, On the macroscopic description of slow viscous flow past a random array of spheres, J. Stat. Phys. 44(5/6), $849-863$ (1986).

[62] J. Rubinstein and S. Torquato, Flow in random porous media : mathematical formulation, variational principles and rigourous bounds, J. Fluid Mech. 206, 25-46 (1989).

[63] P. G. Saffman, On the boundary condition at the surface of a porous medium, Studies Appl. Math. L50, 93-101 (1971).

[64] R. Temam, Navier-Stokes Equations; Theory and Numerical Analysis, North-Holland, Amsterdam (1986).

[65] F. J. Valdés-Parada, C. G. Aguilar-Madera, J. A. Ochoa-Tapia and B. Goyeau, Velocity and stress jump conditions between a porous medium and a fluid, Adv. in Water Res. 62, 327-339 (2013).

[66] S. Whitaker, Flow in porous media I: a theoretical derivation of Darcy's law, Transp. Porous Media 1, 3-25 (1986).

[67] S. Whitaker, The Forchheimer equation: a theoretical development, Transp. Porous Media 25, 27-61 (1996).

[68] S. Whitaker, The Method of Volume Averaging, Theory and Applications of Transport in Porous Media 13, Kluwer Acad. Publ., Dordrecht (1999). 